\title{
Top-down attention: neural pathways in the human and non-human primate examined by \\ electrophysiology, optogenetics and psychophysics
}

\author{
Dissertation \\ for the award of the degree \\ "Doctor rerum naturalium" \\ of the Georg-August-Universität Göttingen \\ within the doctoral program Systems Neuroscience \\ appendant to the Göttingen Graduate School for Neurosciences, \\ Biophysics, and Molecular Biosciences (GGNB) \\ of the Georg-August University School of Science (GAUSS)
}

submitted by

Janina Hüer

born in Lingen (Ems), Germany

Göttingen 2017 
Thesis Committee

Prof. Dr. Stefan Treue

Cognitive Neuroscience Laboratory, German Primate Center, Göttingen, Germany

Prof. Dr. Hansjörg Scherberger,

Neurobiology Laboratory, German Primate Center, Göttingen, Germany

Prof. Dr. Jochen Staiger

Department of Neuroanatomy, Georg-August University, Göttingen, Germany

Members of the Examination Board

Referee: Prof. Dr. Stefan Treue

Cognitive Neuroscience Laboratory, German Primate Center, Göttingen, Germany

$2^{\text {nd }}$ Referee: Prof. Dr. Hansjörg Scherberger

Neurobiology Laboratory, German Primate Center, Göttingen, Germany

Further members of the Examination Board

Prof. Dr. Andrea Antal

Department of Clinical Neurophysiology, University Medical Centre (UMG),

Göttingen, Germany

Prof. Dr. Siegfried Löwel

Systems Neuroscience Group, Johann-Friedrich-Blumenbach Institute for Zoology and Anthropology, Göttingen, Germany

Prof. Dr. Jochen Staiger

Department of Neuroanatomy, Georg-August University, Göttingen, Germany

Prof. Dr. Andreas Stumpner

Department of Cellular Neurobiology, Schwann-Schleiden Research Centre, Göttingen, Germany

Date of oral examination: February 8th, 2018 
Herewith I declare that I have written this thesis independently and with no other aids and sources than quoted.

Janina Hüer 



\section{Acknowledgements}

The work presented in this thesis would not have been possible without the support of other people. First of all, I would like to thank Stefan Treue for supervising me during my thesis, for giving me the opportunity to work in his group and on the projects contained in this thesis. I would like to thank him for all the scientific, but also mental support, and the trust to work on a 'high-risk project'.

I also would like to thank my thesis committee members Hansjörg Scherberger and Jochen Staiger for very helpful discussions and support during the thesis committee meetings, but also beyond that.

I was glad to be part of the optogenetics group of the German Primate Center, which contributed to my projects with very helpful discussions and planning and execution of the surgeries. I would like to say thank you especially to Jens Gruber and Lara Timantra Schiller without whose expertise in virology the implementation and execution of the optogenetics projects would have been much more difficult. I would like to thank Michal Fortuna for support with the neuroanatomy, and, furthermore, Alexander Gail and Igor Kagan for very helpful discussions and suggestions during project presentations. I would like to thank Sonia Baloni for the collaboration in one of the psychophysics projects and Philipp Schwedhelm for fruitful scientific discussions. I would like to say thank you to Lauren Cassidy, Vahid Mehrpour and Benedict Wild for helpful comments on parts of this thesis.

The projects in this thesis required a lot of technical assistance. I am grateful to Leonore Burchardt, Sina Plümer and Dirk Prüße for their help and support and for sharing their wide experience in animal training and handling. I would like to thank Dirk Prüße further for his reliable expertise in surgery-related 
issues and the anesthesia. I am glad that I had support from Ralf Brockhausen in IT-related problems, from Klaus Heisig in the construction of all set-up-related parts and from Beatrix Glaser in administration-related issues. I would further like to thank Luisa Klotz, Janine Kuntze, Ira Panolias, Daniela Trinca Bertazzi Lazzarini, Natalie Bobb and Patricia Sprysch for technical assistance during my projects, and Artur Kaul and Gerhard Hoch for helpful discussions about biosafety and laser safety during the implementation phase of the optogenetics projects. I would like to thank all the animal caretakers for taking care of the animals with a lot of dedication and creativity.

I am glad to have had the best office mates Dana, Lauren and Amr during the time of my thesis.

Last but not least, I would like to thank the most important basis for conducting this work: my family and friends, especially Vicky, who were always there in the good and bad phases with a lot of support and patience. 


\section{Contents}

1 Introduction $\quad 1$

1.1 Part I . . . . . . . . . . . . . . . 5

1.1.1 Visual processing pathways ........... 5

1.1.2 The medial temporal area . . . . . . . . . . . . . 6

1.1.3 Attentional modulation in area MT . . . . . . . . 9

1.1.4 The premotor theory of attention . . . . . . . . . 12

1.1.5 Anatomical and physiological classification of the FEF 14

1.1.6 Attentional modulation in the FEF . . . . . . . . 16

1.1.7 Evidence for the guidance role of the FEF in attention 17

1.1.8 Anatomical connections between the FEF and area MT 19

1.1.9 Optogenetic targeting of the projection from FEF to area MT . . . . . . . . . . . . . 20

1.2 Part II . . . . . . . . . . . . . . . . . . . 23

1.2.1 The attentional blink . . . . . . . . . . . . 23

1.2.2 The locus coeruleus . . . . . . . . . . . . . . 25

1.2.3 The effect of attention on pupil size . . . . . . . 25

2 Project manuscripts $\quad 29$

2.1 An anatomical viability evaluation of using optogenetics to examine the fronto-visual and fronto-parietal network in the macaque . . . . . . . . . . . . . . . . . . 31

2.2 Optogenetic inhibition of FEF input to area MT reduces attentional modulation of neuronal responses . . . . . . . . 47

2.3 An attentional blink with motion stimuli and in a task combining motion and letter stimuli . . . . . . . . . . . . . . . . . . 69 
2.4 Pupil size suggests that the locus coeruleus is involved during attentional blink tasks without a direct perceptual consequence

$\begin{array}{lll}3 & \text { Summary and outlook } & 99\end{array}$

$\begin{array}{ll}\text { Bibliography } & 103\end{array}$

$\begin{array}{ll}\text { Supplementary material } & 131\end{array}$ 
"A pure sensation is an abstraction; [...]"

"Pure sensations can only be realized in the earliest days of life.

They are all but impossible

to adults with memories and stores of associations acquired."

(William James, 1980) 



\section{Chapter 1 \\ Introduction}

We are constantly exposed to an abundance of sensory information in our environment. Our brain has to deal with this information. It has to filter out the most relevant information and process it to guide our behavioral actions. We are not automatically driven and guided by the most salient input in our environment. Instead, we are able to voluntarily attend to selected information. Top-down mechanisms of selective attention help us to guide our behavior despite continuous bottom-up sensory input.

The term 'selective attention' describes the process of attending to selected information, and can be contrasted, for example, to arousal, which describes the overall attentiveness of an individual. Attention is classically divided into exogenous attention (or automatic or bottom-up attention), in which highly salient information captures our processing resources in a reflexive way, and endogenous attention (or voluntary or top-down attention) that can be deliberately deployed to selected information. The proper balance between these two forms of attention is crucial to our ability to behave in a goal-directed manner, yet still remain flexible and responsive to unexpected but important events in our environment.

There are several circumstances in which the control of our attentional system is unbalanced. The so called attention-deficit/hyperactivity disorder (ADHD) is a complex, heterogenous condition, in which individuals show impairments in cognitive and attentional everyday tasks (criteria are defined, e.g., by the American Psychiatric Association). ADHD affects around 5\% of humans worldwide (Polanczyk et al., 2007). Current treatments include the administration of drugs like amphetamine, methylyphenidate, or atomoxetine (Briars 
and Todd, 2016; Mueller et al., 2017) - drugs that directly change dopamine and noradrenaline signaling in the brain. The neurotransmitters dopamine and noradrenaline naturally arise in subcortical structures of the brain, from where dopaminergic and noradrenergic neurons innervate a majority of brain structures via widespread axonal projections (e.g., Levitt et al., 1984; Morrison et al., 1982).

One of the processes that can be impaired in ADHD patients is selective attention (Mueller et al., 2017). Neural networks implicated in selective attention, e.g., the frontoparietal network, have been suggested to show altered or dysregulated activity in ADHD patients (for a review see Bush, 2010; Castellanos and Proal, 2012). Dopamine and noradrenaline can directly affect these neural networks (for a review see Clark and Noudoost, 2014). For example, it has been shown that injection of a synthetic dopamine antagonist into the prefrontal cortex of macaques influences the top-down signals that this area sends to extrastriate visual cortex (Noudoost and Moore, 2011a).

However, we have only begun to understand how the networks underlying selective attention function under normal conditions. This understanding is crucial for identifying how network interactions differ in ADHD. The neural mechanisms cannot be fully investigated in human patients as their brains are only limitedly accessible. Therefore, non-human primates are an important animal model to identify the neural circuits and processes controlling selective attention. Only with a profound understanding, proper treatment for the variety of ADHD symptoms can be developed.

This thesis deals with the processes underlying selective endogenous attention in the visual system of the human and non-human primate. Within endogenous attention there is a classical differentiation between overt and covert attention. Overt attention is the most intuitive form of attention as it describes attention to visual information at the center of gaze. But attention can also be deployed covertly ${ }^{1}$ (James, 1890; Sperling and Melchner, 1978; Posner, 1980).

\footnotetext{
${ }^{1}$ It should be mentioned at this point that recent psychophysical results indicate that covert attentional mechanisms also act at the very center of gaze, that is, not to the center of gaze but to the periphery of the visual field (Poletti et al., 2017), and therefore the classical differentiation might need a reconsideration.
} 
In addition, top-down attention can be directed to a position in space (spatial attention), a specific stimulus feature, e.g., color or motion direction, (featurebased attention) or whole objects (object-based attention). These three forms of attention interact with each other and often cannot be fully disentangled.

In the scope of this thesis, the processes underlying top-down attention have been examined in the dorsal visual processing stream by using different methods. The first part of this work explores the role of the frontal eye field (FEF), a part of the prefrontal cortex, in mediating top-down spatial attention effects in the visual medial temporal area (area MT). We used optogenetics in the rhesus macaque and conducted two experiments. One of the objectives of the first experiment was to deliver histological evidence that the direct anatomical connection from the FEF to area MT can be targeted with optogenetics. In the second experiment, we optogenetically inhibited this connection while a monkey was performing a spatial attention task. Results of previous studies, e.g., Gregoriou et al. (2014), have indicated that the connection from the FEF to extrastriate visual areas is relevant during the deployment of top-down attention. However, our experiment delivers the first evidence that the FEF sends information via this direct anatomical connection to an extrastriate visual area during top-down attention. In addition, ours is the first study that probes the role of the FEF in mediating attentional effects in area MT, since previous studies mainly targeted visual area V4.

In the second part of this thesis, we explored the limitations of our top-down attentional system, apparent during a phenomenon known as the attentional blink. Here, we first conducted a series of experiments in human subjects to prove that an attentional blink occurs for moving stimuli, which are processed by the dorsal visual pathway. Previous studies mainly used stationary visual stimuli, which are processed by the ventral visual pathway. In a second experiment, we measured pupil size in addition to psychophysical performance during a similar behavioral task. We reconstructed a sequence of attentional input that potentially underlies the changes in the pupil size by using a previously developed model (Hoeks and Levelt, 1993; Wierda et al., 2012) and related it to the behavioral conditions. Pupil size and attentional traces can be linked to the activity of a subcortical structure in the brain stem, the locus 
coeruleus (LC), which provides the main noradrenaline input to the cortex (Berridge and Waterhouse, 2003). The LC has been hypothesized to play a role during the attentional blink (Nieuwenhuis et al., 2005), and our study delivers evidence that this might indeed be the case.

Due to the higher scientifc impact of the first two projects, the first part of this thesis is elaborated in more detail. 


\section{$1.1 \quad$ Part I}

\subsubsection{Visual processing pathways}

In a very simplified depiction, visual information received by the retina is transmitted to the lateral geniculate nucleus of the thalamus and from there to the first visual cortical area V1. Along this processing stream up to V1, basic qualities of visual information, for example, color, motion, and contrast, are processed seperately (reviewed in e.g., Livingstone and Hubel, 1988; Nassi and Callaway, 2009).

In V1, two main visual processing streams emerge - a dorsal and a ventral pathway. The idea of the existence of two different processing streams stems from anatomical, physiological, and lesion studies. Two of the earliest studies suggesting that different features of visual information are processed separately in the cortex were conducted by Newcombe and Russell (1969) and Pohl (1973) on human subjects with lesions in distinct brain regions. Both studies showed that lesions in parietal areas cause deficits in spatial localization experiments, while lesions in the temporal lobe are associated with an impairment in visual-perceptual tasks. These among several other studies inspired Ungerleider and Mishkin (1982) to formulate the hypothesis that a ventral visual stream is responsible for object perception ('what'), while a dorsal visual stream is analyzing spatial information ('where').

Within this framework, sensory input is processed in the visual system via a hierarchical order of visual areas (e.g., Felleman and Van Essen, 1991; Nassi and Callaway, 2009). Processing along the dorsal visual pathway starts in V1, includes V2 and V3, and continues in area MT. Area MT sends information to higher areas like MST, FST, VIP, LIP and 7a. Processing along the ventral visual pathway starts in V1, includes V2, V3, and continues to V4. From there information is transferred, i.a., to the subdivisions PIT, CIT and AIT of the inferotemporal cortex. Both processing streams converge again in the FEF (Bullier et al., 1996).

The two stream hypothesis has been reviewed by Goodale and Milner (1992), who suggested that it is more plausible not to describe the pathways by means of the type of information that is processed, but rather by the aim the informa- 
tion is processed for. According to this hypothesis, the ventral visual stream is responsible for object perception and identification ("what'), while the dorsal visual pathway is conducting processing that is necessary for visually guided actions on those objects ('how'). This reconsideration did not affect the attribution of the brain areas to the two pathways.

The distinction between two processing streams is by no means rigid and exclusive. There is interaction between the areas assigned to the two pathways (for review see Cloutman, 2013), and a recent study suggests that there are even three pathways in the human (Haak and Beckmann, 2017). The general idea of two main visual pathways, however, has been proven as a feasible simplification of visual processing until now.

\subsubsection{The medial temporal area}

Area MT lies in the posterior bank of the superior temporal sulcus. First anatomical descriptions in the rhesus macaque stem from Zeki (1969) and Cragg (1969), who showed that this area of the cortex receives afferents from area V1. Allman and Kaas (1971) coined the name 'medial temporal area' when they described the area in the owl monkey. Area MT can be anatomically differentiated from its neighboring areas since it contains an area of heavy myelination of mainly layers IV, V and VI, and by its direct input from V1 (Zeki, 1974; Van Essen et al., 1981; Albright and Desimone, 1987). Its surface was estimated to be between $33 \mathrm{~mm}^{2}$ (Van Essen et al., 1981) and $80 \mathrm{~mm}^{2}$ (Albright and Desimone, 1987; Gattass and Gross, 1981), and therefore its size is around 5\% the size of V1 (Felleman and Van Essen, 1991).

Area MT contains neurons that respond selectively to visual motion (Dubner and Zeki, 1971; Zeki, 1974; Van Essen et al., 1981; Maunsell and Van Essen, 1983; Albright, 1984; Albright et al., 1984). As an example, Desimone and Ungerleider (1986) found 89\% of neurons in MT to be direction-selective (that means the neurons' firing rate differed reliably between the presentation of two opposing motion directions). Therefore, the lateral border of MT can be easily determined physiologically, as the lateral neighboring cortex (i.e., V4 and V4t) is not rich in direction-selective cells (Van Essen et al., 1981; Desimone and Ungerleider, 1986). Desimone and Ungerleider estimated the percentage of direction-selective cells to be $25 \%$ in V4t and $15 \%$ in V4. Neurons at the 
medial side (i.e., area MST) show direction selectivity, but have bigger receptive fields (Van Essen et al., 1981). Receptive fields of MT neurons can have a diameter of up to 50 degrees of visual angle (dva) (Felleman and Kaas, 1984; Gattass and Gross, 1981). They are usually much smaller closer to the fovea and increase with eccentricity. MT receptive field sizes are a factor of 10 bigger than receptive fields in V1 (Albright and Desimone, 1987).

Presentation of a moving stimulus within their receptive field increases the firing rate of MT neurons dependent on the shown movement direction. Neurons typically have a preferred direction and respond less to other movement directions. Maunsell and van Essen (1983) calculated the ratio between response to the preferred direction and response to the non-preferred (opposite) direction to be around 11. The firing rate dependence on movement direction can be fitted with a gaussian tuning curve (Albright, 1984) and typically exhibits on average a bandwidth of around $90^{\circ}$ (Albright, 1984).

The spatial extent of receptive fields of MT neurons is not perfectly circular or gaussian and homogenous concerning their preferred direction. Instead, fine-scaled analysis have shown that many neurons in area MT have multiplepeaked receptive fields or varying preferred directions across their receptive field (Richert et al., 2013). In addition, facilitatory and suppressive interactions have been found to act within the receptive field (Livingstone et al., 2001). Many neurons in MT show a center-surround structure (Xiao et al., 1997), whose strength can be contrast-dependent (Tsui and Pack, 2011), and which is not necessarily circular and symmetric (Xiao et al., 1997).

Besides movement direction, neurons in MT respond selectively to movement speed (Maunsell and Van Essen, 1983; Rodman and Albright, 1987; Lagae et al., 1993; Perrone and Thiele, 2001) and disparity (Maunsell and Van Essen, 1983; Bradley et al., 1995; DeAngelis and Newsome, 1999). Furthermore, many neurons show orientation selectivity (Maunsell and Van Essen, 1983; Albright, 1984); responses are often best to a stimulus orientation perpendicular to the preferred direction (Maunsell and Van Essen, 1983; Albright, 1984). Neurons in MT are usually not selective for color (Thiele et al., 1999) or stimulus form (Albright, 1984).

As in many visual areas, MT neurons are retinotopically organized (Zeki, 
1974; Van Essen et al., 1981; Albright and Desimone, 1987), although the order can vary from individual to individual (Van Essen et al., 1981; Desimone and Ungerleider, 1986). The central 15dva can occupy around half of the area of MT (Van Essen et al., 1981). In addition, MT neurons show a columnar organization concerning their direction selectivity: neighboring neurons have similar preferred movement directions (Dubner and Zeki, 1971; Van Essen et al., 1981; Maunsell and Van Essen, 1983; Albright et al., 1984). Albright and colleagues showed that $180^{\circ}$ of axis of motion are represented in around $500 \mu \mathrm{m}$ of cortex (Albright et al., 1984). Within this organization, jumps of $180^{\circ}$ changes of preferred movement direction have been reported (Maunsell and Van Essen, 1983; Albright et al., 1984).

In the visual hierarchy, area MT is assumed to be higher than areas V1, V2 and V3. This assumption is based on the connectivity pattern of the reciprocal connections with these areas (Rockland and Pandya, 1979; Markov et al., 2013; Maunsell and van Essen, 1983; Felleman and Van Essen, 1991).

Projections from MT to V1, V2 and V3 end in supra- and infragranular layers, while projections from V1, V2 and V3 arise in supragranular layers (Maunsell and van Essen, 1983; Ungerleider and Desimone, 1986). In the case of the reciprocal connection of MT and V1 it has been shown that while projections from V1 arise mainly in layer IVB and layer VI, the projections from MT to V1 target exactly those two layers (Maunsell and van Essen, 1983). Area MT is assumed to be on a similar hierarchical level than area V4, since projections from MT to V4 end in all cortical layers, and projections from V4 to MT originate in supra- and infragranular layers (Ungerleider and Desimone, 1986). Areas MST, FST and VIP are higher in the visual hierarchy. Projections from area MT end in layer 4 of these areas, while projections from these areas to MT originate in supra-and infragranular layers (Maunsell and van Essen, 1983; Ungerleider and Desimone, 1986).

Due to the characteristics of MT neurons it is not surprising that several studies in the non-human primate revealed a role for area MT in motion perception. The first supporting evidence for this role was obtained in a study of Newsome and colleagues, who found that small lesions in area MT affected 
motion processing (Newsome et al., 1985). Monkeys with a lesion had difficulties in adapting their eye movements to the movement of a visual stimulus shown on a screen in front of them. In contrast, responses to a stationary stimulus were unaffected. Albeit this experiment established a first link between motion processing and area MT, it did not directly measure the consequences of an MT lesion on motion perception. Newsome and Paré measured the effects of MT lesions on psychophysical performance of monkeys (Newsome and Paré, 1988). They found that motion thresholds were clearly elevated with a lesion, i.e., monkeys needed a stronger motion signal to correctly discriminate a motion direction. Hence, the perception of the stimulus motion appeared to be directly affected by a lesion. In line with that are results of Salzman and colleagues, who compared psychophysical performance of monkeys with the performance of MT neurons in a direction discrimination task (Salzman et al., 1992). Their results suggest that only a small number of MT neurons was necessary to account for the perceptual performance of the monkeys in their study. Furthermore, the behavioral choice of monkeys was correlated with the trial-to-trial variability of MT responses independent of the effects of visual stimulation in a motion discrimination task (Britten et al., 1996). Taken together, these results provide strong evidence that neural activity in area MT is directly contributing to motion perception.

\subsubsection{Attentional modulation in area MT}

Attention modulates neural activity in a multitude of areas in the visual system of the macaque. Modulations have been observed as early as the lateral geniculate nucleus (McAlonan et al., 2008; O'Connor et al., 2002) and have been found in areas such as V1 (Motter, 1993; McAdams and Maunsell, 1999), V2 (Motter, 1993; Luck et al., 1997), V4 (Moran and Desimone, 1985; Motter, 1993; Luck et al., 1997; McAdams and Maunsell, 1999), IT (Moran and Desimone, 1985), and VIP (Cook and Maunsell, 2002).

After first studies failed to find attentional effects on MT activity (Ferrera et al., 1994; Newsome et al., 1988), Treue and Maunsell showed that neural responses in area MT can depend on the behavioral relevance of a motion stimulus (Treue and Maunsell, 1996; Treue and Martinez Trujillo, 1999). In compliance with the findings that activity of MT neurons contributes to the 
perception of motion, hence, higher cognitive processes adjust this activity dependent on the current behavioral demands.

Treue and Maunsell (1996) used a design whose general logic has been used in similar ways in other studies (e.g., Motter, 1993) and that can be described as follows. Two monkeys were performing a spatial attention task while the authors recorded single-neuron activity in area MT. The animals had to foveate a fixation point in the center of a computer screen. Consequently, two moving dots were shown to the animals, one in the receptive field of the recorded neuron, the other dot outside of it. The dots always moved in the preferred direction of the neuron. One of the dots was presented earlier to the monkeys, and this identified the respective dot as the target stimulus. Both dots were moving continuously and the monkeys had to respond to a speed change of the target stimulus, while ignoring speed changes of the distractor dot. In this way, the attentional state of the monkeys was manipulated while keeping the sensory input identical. The authors found that firing rates of MT neurons were on average increased by $19 \%$ when the monkeys attended to the stimulus in the receptive field compared to when they attended to the stimulus outside of the receptive field - an effect that can only be attributed to spatial attention. In a second experiment, Treue and Maunsell presented an additional dot in the receptive field, moving in the opposite, non-preferred direction. In this case, firing rates were dependent on which of the two dots within the receptive field was attended. Responses were on average $86 \%$ higher when the dot moving in the preferred direction was the target stimulus compared to when the dot moving in the non-preferred direction was the target. When attention was directed to the dot outside of the receptive field, the neurons exhibited an intermediate response.

Taken together, these results showed that MT neurons are modulated by spatial attention, and indicated that activity is also dependent on other features (i.e., movement direction) of an attended stimulus.

Additional studies found attentional effects in area MT, which were sometimes lower than the enhancement described above. Seidemann and Newsome, for example, reported that spatial attention increased responses of MT neurons by only $9 \%$ on average when monkeys attended to one of two stimuli moving in opposite directions within the receptive field (Seidemann and Newsome, 
1999). Treue and Maunsell (1996) found an $86 \%$ increase of firing rates in this condition. Seidemann and Newsome argued that the effects found by Treue and Maunsell must be due to additional forms of attention besides spatial attention. However, several other parameters in the task designs could also explain the differing results; the most relevant presumably being that in one study animals had to monitor the target stimulus continuously (Treue and Maunsell, 1996), while in the other study animals had to discriminate the direction of a target stimulus (Seidemann and Newsome, 1999). Discrimination does not necessitate prolonged monitoring of the stimulus, and therefore animals might just use a limited time period of stimulus presentation or withdraw their attention as soon as they accumulated enough information about the motion direction of the stimulus.

Recanzone and Wurtz found that activity of MT neurons was enhanced with attention in the receptive field by $50 \%$ in a task that demanded the animals to conduct pursuit eye movements (Recanzone and Wurtz, 2000). Attentional effects were time dependent. If the monkeys ${ }^{6}$ attention was in the receptive field and the stimuli started moving $450 \mathrm{~ms}$ earlier, activity was enhanced. In contrast, little modulation occurred when the stimuli started moving only $150 \mathrm{~ms}$ earlier. A time-dependence of attentional modulation has also been reported in other studies in area MT (Treue and Maunsell, 1999; Seidemann and Newsome, 1999), and also V4 (McAdams and Maunsell, 1999).

The effect of spatial attention on MT neurons appeared to be a multiplicative enhancement of responses to all movement directions, without changing the tuning width of the neurons (Treue and Maunsell, 1999; Recanzone and Wurtz, 2000). A similar effect has been reported for V4, where spatial attention enhanced firing rates in a multiplicative manner, leaving the width of the orientation tuning curve unaffected (McAdams and Maunsell, 1999). This multiplicative effect on the tuning curve resembles the effect that an increase of stimulus contrast has on neural responses, e.g., in area V1 of the cat (Sclar and Freeman, 1982) and suggests that the effect of attention is similar to an increase of stimulus intensity.

As indicated above, neuronal responses in area MT are also modulated by feature-based attention (Treue and Maunsell, 1999). Attending to a preferred direction outside of the receptive field increased firing rates by on average $13 \%$ 
compared to attention to the non-preferred direction. Treue and Martinez Trujillo formulated the 'feature-similarity gain model' according to which the gain of an MT neuron is modulated depending on how the characteristics of an attended stimulus, be it location or direction, matches the preferred characteristics of a neuron. Spatial location, in this model, is just a feature like direction or orientation.

However, results of experiments presenting two stimuli within the receptive field contradict the assumption that attention acts by a simple gain change of the neurons. For example, Martinez-Trujillo and Treue showed that the attentional modulation in MT depended non-linearly on stimulus contrast of an unattended stimulus within the receptive field: Attentional modulation was higher for intermediate stimulus contrasts compared to low and high contrasts (Martínez-Trujillo and Treue, 2002). These findings together with the predictions of the feature-similarity gain model can, however, be explained by a normalization mechanism of attention, which acts on the input converging in neurons of MT or other visual areas, rather than directly changing the neurons firing rates (e.g., Lee and Maunsell, 2009; Reynolds and Heeger, 2009; Lee and Maunsell, 2010). The normalization can explain multiplicative as well as non-linear effects, as it assumes that attention not only enhances excitatory effects on the cells, but also inhibitory. Therefore, the attentional effects depend on the exact stimulus configuration as well as the attentional requirements.

Spatial attention can also shift receptive fields in the direction of an attended location (Womelsdorf et al., 2006; Anton-Erxleben et al., 2009), or expand receptive field sizes during covert attentive tracking of a stimulus (Niebergall et al., 2011).

The reliable finding of attentional effects in extrastriate visual areas poses the question where the attentional modulation originates.

\subsubsection{The premotor theory of attention}

The premotor theory of attention is probably the most influential theory to explain the neural mechanisms underlying top-down attention (Rizzolatti et al., 1987; Craighero and Rizzolatti, 2005). It claims that the neural circuit active during the deployment of attention is the same as the circuit active during 
the execution of eye movements. According to the theory, these two forms of behavior differ only in the strength of network activation, but not in the composition of the network per se. Directing covert attention to a point in space, therefore, is similar to the preparation of an eye movement to that point; the difference between the two behaviors being only that during covert attention the execution of eye movements is inhibited on a peripheral level.

A meta-analysis of imaging studies indicated that similar neuronal networks are activated during attention and eye movements, but the analysis also suggested a partial segregation of the networks (Corbetta, 1998). Therefore, Corbetta and colleagues directly compared the two types of behavior by using functional magnetic resonance imaging (fMRI) and surface-based representations of functional brain activity in human subjects (Corbetta et al., 1998). They contrasted active brain areas in a task that required subjects to shift attention either with or without eye movements. Both forms of behavior activated a largely overlapping network composed of parietal, frontal (putative FEF and SEF) and temporal regions, and the authors concluded that attention and eye movements indeed use the same network. These results were subsequently confirmed by other imaging studies (e.g., Nobre et al., 2000; Astafiev et al., 2003).

Based on these findings, a series of studies has been conducted in the nonhuman primate to explore the neural mechanisms underlying attention and eye movements (e.g., Moore and Armstrong, 2003). Moore and Armstrong, for this purpose, targeted visual area V4, in which firing rates are modulated by attention (e.g., Moran and Desimone, 1985), and an area involved in oculomotor processing, the FEF (e.g., Bruce and Goldberg, 1985; Corbetta et al., 1998). The authors hypothesized that if the oculomotor system is involved in the attentional modulation observed in visual areas, stimulation of the selfsame system should result in similar enhancements of firing rates in visual areas as attention does. And this is indeed what the authors found: firing rates in area V4 were enhanced by electrical microstimulation of FEF neurons similar to the enhancement found with attention.

Several subsequent studies showed a link between the FEF and attention and eye movements (e.g., Moore, 2004; Wardak, 2006; Noudoost and Moore, 2011a). Due to these findings, the FEF is considered to be one of the key areas 
in spatial attention and the accompanied modulation of firing rates in visual areas. Before we come to the results of these studies, the FEF is described in more detail in the following sections.

\subsubsection{Anatomical and physiological classification of the FEF}

The FEF can be found in the human as well as the non-human primate. Unfortunately, the anatomical assignment of labels to the FEF and the use of them is not consistent among researchers. The FEF lies in the part of the prefrontal cortex that has been numbered as area 8 by Brodmann (1909). As depicted by Walker (1940), this area does not appear to be similar in humans and non-human primates when comparing cytoarchitectural characteristics. It can be divided into area $8 \mathrm{~A}$ which runs along the arcuate sulcus, and area 8B which extends into the medial direction. Area 8A has large pyramidal cells in layer 5 , whereas area $8 \mathrm{~B}$ does not. Both areas can be characterized by the presence of a thinner layer 4 compared to more anterior areas. Some researchers described the FEF as spanning over area $8 \mathrm{~A}$ and the neighboring area 45 (e.g., Bruce and Goldberg, 1985; Schall, 2009; Rockland et al., 2013). However, it has been argued that microstimulation of area 45, when classified with the help of human criteria, does not evoke eye movements (Petrides et al., 2005, 2012).

What can be summarized is that the FEF lies in the rostral bank of the arcuate sulcus. From the point where a posterior extension of the principal sulcus would meet the arcuate sulcus, the FEF extends into lateral, medial and caudal direction along the arcuate sulcus.

The FEF has first been described by Ferrier (1874) who showed that electrical microstimulation of the frontal lobe of the brain can evoke eye movements to the visual space contralateral to the stimulated hemisphere. Several early studies replicated this finding (e.g., Beevor and Horsley, 1888; Russell, 1894), and common agreement exists that the FEF can be classified physiologically by means of eliciting eye movements with microstimulation.

Bruce and Stanton (1985) and Bruce et al. (1985) systematically investigated 
the physiological characteristics of the FEF in rhesus macaques. They defined the FEF as the area in which microstimulation with less than $50 \mu \mathrm{A}$ evokes eye movements. Under that classification, they found that the presence of large pyramidal neurons in layer 5 is a reliable determinant to confine the FEF anatomically.

The FEF has been divided into two parts based on the length of evoked saccades. Shorter saccades are generated by lateral FEF and longer saccades by medial FEF (Robinson and Fuchs, 1969; Bruce and Goldberg, 1985).

Many neurons in the FEF can be characterized as belonging to one of three different groups: visual neurons, movement neurons and visuomovement neurons (e.g., Mohler et al., 1973; Bruce and Goldberg, 1985; Schall, 1991; Sato and Schall, 2003). This classification is based on how the neurons respond to visual input and saccade execution, and is usually described during a saccade task. Visual neurons, as the name suggests, respond mainly to visual sensory input, for example, a saccade target, but not to saccade execution (Bruce and Goldberg, 1985). Receptive fields have been reported to extend to 40dva of diameter, however, they were often described to cover a quarter of the used computer screen in the studies and, therefore, reported sizes might underestimate the actual size (e.g., Mohler et al., 1973; Kodaka et al., 1997; Cavanaugh et al., 2012). Movements cells do not or only weakly respond to visual stimulation, but show enhanced firing rates before or after the onset of saccades (Mohler et al., 1973; Bruce and Goldberg, 1985). Instead of a visual receptive field, they have a movement field, which analogously describes the area in space to which planning or execution of a saccade evokes a response in the neuron (Bruce and Goldberg, 1985). Visuomovement cells usually exhibit characteristics in between the two types of cells, and there seems to be a continuum from visual to movement cells (Bruce and Goldberg, 1985). In the following, the term 'response fields' is used to jointly refer to visual receptive fields and movement fields of FEF neurons whenever a differentiation is not adequate.

Cohen and colleagues found that the three types of neurons can also be classified by distinct biophysical characteristics: movement neurons have the widest action potential waveforms, and visual neurons have wider spikes than visuo- 
movements neurons (Cohen et al., 2009). Due to their thin spikes, visuomovement cells have been suggested to be inhibitory (Cohen et al., 2009).

In addition to these three cell-types, there are fixation neurons in the FEF (e.g., Izawa et al., 2009; Izawa and Suzuki, 2014), which discharge during active fixation. Also within this group of neurons, there seems to be a continuum of cells responding to the visual input of the fixation point to cells responding due to the active motor process of fixation (Izawa et al., 2009).

Neurons in the FEF are thought not to be feature-selective. Mohler et al. (1973), for example, did not find movement, direction or orientation selectivity. Under certain circumstances, FEF neurons can show selectivity for stimulus features, like color (Bichot et al., 1996).

However, FEF neurons are tuned for direction and amplitude of saccades. This is true for all types of neurons, though visual cells show the narrowest tuning (Bruce and Goldberg, 1985). Therefore, neurons in the FEF might be best understood in terms of representing stimuli by means of executing a potential saccade movement to the stimulus instead of representing the absolute position of stimuli.

\subsubsection{Attentional modulation in the FEF}

Similar to area MT, a first study failed to find changes related to covert attention in the FEF (Goldberg and Bushnell, 1981). However, later studies consistently found attentional effects in the FEF. Kodaka and colleagues recorded activity in the FEF during a simple spatial attention task (Kodaka et al., 1997). Monkeys had to detect a luminance change either of a peripheral target stimulus within the receptive field of a recorded neuron or of the fixation point. By comparing firing rates between these two conditions, the authors found that $51 \%$ of visually responsive neurons (visual and visuomovement cells) were modulated by attention. The modulation was not always an increase in firing rates (often attention suppressed responses), but on average attention enhanced firing rates by $38 \%$.

Several subsequent studies reported that visual and visuomovement cells are modulated by attention; the neurons usually showed an enhancement of firing rates (Thompson, 2005; Khayat et al., 2009; Gregoriou et al., 2012). In contrast, movement cells were either inhibited or not affected by attention. 
As the result of a modeling study, Hamker (2005) suggested that movement cells provide the best signal for driving attentional effects in visual areas. However, the results of the experimental studies indicate that it is not the motor neurons that cause attentional modulation of neurons in visual areas. Instead, the results suggest that only visual and visuomovement cells play a role during the deployment of attention.

\subsubsection{Evidence for the guidance role of the FEF in at- tention}

Moore and Fallah were the first to test whether stimulation of the oculomotor network in the FEF plays a role in attention (Moore and Fallah, 2001). They trained monkeys to perform a spatial attention task: a luminance change in a target stimulus had to be detected and distractor stimuli presented at other spatial locations had to be ignored. While the animals were conducting the task, the authors electrically stimulated the FEF. The stimulation current was below the strength that would evoke eye movements. The authors found that when the target stimulus was presented within the response field of the stimulated FEF neurons, the performance of the animals was improved. These results indicated that the FEF network that is involved in evoking eye movements, is also involved during visual attention.

Moore and Armstrong used a similar approach of microstimulation in the FEF, but examined the effect on neural activity in area V4 (Moore and Armstrong, 2003). Monkeys did not perform a visual attention task, but instead were fixating a fixation point. During fixation, one oriented bar was presented on the screen, either in the receptive field of the recorded V4 neuron or outside of it, and either in the preferred or non-preferred orientation. Stimulation electrodes were placed in the vicinity of FEF neurons having either overlapping response fields with the V4 receptive field, or non-overlapping response fields. FEF stimulation of overlapping response fields caused an increase in V4 neurons' firing rates when the preferred stimulus was shown in the receptive field, an intermediate increase when a non-preferred stimulus was shown, and no increase when no stimulus was shown. In a second experiment, the authors tested how the presence of a distractor stimulus affects the stimulation 
results. They found that stimulation effects were higher when the distractor was shown compared to when it was absent. In addition, stimulation increased firing rates when the stimulated FEF response fields were overlapping with the $\mathrm{V} 4$ receptive field and the preferred stimulus was shown in the V4 receptive field. In contrast, stimulation resulted in a suppression of V4 responses when FEF neurons with non-overlapping response fields were stimulated and the preferred stimulus was shown. The authors found an average enhancement of firing rates of $20 \%$ comparing these two conditions, which, as they argue, is similar to the enhancement found in spatial attention studies (e.g., McAdams and Maunsell, 1999).

Results gained with electrical microstimulation have limitations in their interpretability. Electrical microstimulation not only stimulates neuronal cell bodies in the vicinity of the stimulation electrode. It also stimulates fibers of passage and axons projecting to the stimulation area, and, thus, can antidromically activate the corresponding neurons. Consequently, neurons of other areas can be stimulated during electrical microstimulation (see, e.g., Histed et al., 2013).

This limitation has been overcome in a study of Noudoost and Moore (2011a). The authors changed the dopamine-mediated activity in FEF by local pharmacological injection of the dopaminergic antagonist SCH23390. This antagonist acts on the D1 receptors, and has been shown to increase working memory related activity within the FEF (see Noudoost and Moore, 2011a). Monkeys were conducting a free-choice saccade task. Injection of SCH23390 increased the tendency of the monkeys to choose the saccade target that was presented within the response field of the FEF neurons affected by the pharmacological injection. The authors recorded neural activity in area V4. Activity of V4 neurons was enhanced by the injection of SCH23390 when the receptive fields of the V4 neurons were overlapping with the response fields of the affected FEF neurons. Even though the experiment did not show a role of dopamine in attention, it demonstrated that the FEF can mediate changes in firing rates in area V4 without the confound of stimulating axons from other areas.

The most direct evidence that the FEF plays a role in attention stems from a study of Gregoriou and colleagues and shows a causal relationship of activity 
in the prefrontal cortex and attentional modulation in area V4 (Gregoriou et al., 2014). The authors lesioned the prefrontal cortex, including the FEF, of one hemisphere and evaluated the effect on attentional modulation of V4 neurons. Attentional modulation of firing rates was reduced by on average $40 \%$ in V4 of the lesioned hemisphere compared to V4 of the non-lesioned hemisphere. In addition, latencies of attentional effects were longer.

Several other studies showed a link between activity in the FEF and either firing rates in visual area V4 (Moore, 2004; Armstrong and Moore, 2007; Gregoriou et al., 2009, 2012; Noudoost et al., 2014; Merrikhi et al., 2017) or during attention (Wardak, 2006; Rossi et al., 2007).

\subsubsection{Anatomical connections between the FEF and area MT}

As many cortical areas do, the FEF and area MT possess reciprocal connections (e.g., Tigges et al., 1981). Stanton et al. (1995) showed that area MT primarily receives input from the small saccade part of the FEF. Neurons projecting to area MT are located in supra- and infragranular layers of the FEF (60\% in supragranular layers, Ninomiya et al., 2012). Two differing results have been found concerning their projection localization in area MT. Stanton et al. (1995) reported a columnar distribution in area MT, that is, all layers received input from the FEF (this was different to other areas, where projections ended mainly in layer I and V/VI). Leichnetz (1989), in contrast, found that projections mainly end in layer V and VI of area MT.

There is more unity for the results showing the origin of MT projections to the FEF. Leichnetz et al. (1989) found that MT neurons projecting to the FEF are mainly localized in layer III of area MT. Similar results have been reported by Huerta et al. (1987), who detected MT cells projecting to the FEF mainly in supragranular layers. Schall et al. (1995) showed that MT neurons projecting to the FEF are mainly located in layer III, but they also found cells in infragranular layers. The projection from area MT to the FEF appears to be topographic. The medial FEF (i.e., the large saccade part of the FEF) receives innervations from peripheral visual fields in area MT, while the lateral FEF (i.e., the small saccade part of the FEF) receives innervations 
from the central visual field of area MT (Schall et al., 1995).

The projection from the FEF to area V4 has been investigated in more detail. Since area V4 and area MT are considered to be on a comparable hierarchical level in the visual system, these results could be similar for the FEF projection to area MT and are therefore mentioned here. However, it should be noted that different populations of FEF neurons project to areas MT and V4 (Ninomiya et al., 2012). Therefore, the results cannot be transferred to the FEF projection to area MT without reservation.

Anderson and colleagues showed that FEF neurons projecting to area V4 exhibit the characteristics of excitatory pyramidal cells and project predominantly to excitatory cells in area V4 (Anderson et al., 2011). A minority of targets were inhibitory neurons $(2 \%)$. FEF neurons projecting to area V4 were predominantly found in supragranular layers of the FEF (Pouget, 2009; Barone et al., 2000), and classified as feedforwark connection (Barone et al., 2000). In contrast, neurons projecting to the superior colliculus, an area of the oculomotor system, were only found in layer 5 , and none of the neurons in layer 5 that projected to the superior colliculus also projected to area V4 (Pouget, 2009). Therefore, distinct populations of neurons seem to project to area V4 and the superior colliculus. Segraves and Goldberg (1987) showed that a majority of neurons projecting to the superior colliculus were movement cells and located in layer $\mathrm{V}$ of the FEF. These results are interesting, as they indicate that movement cells that are involved in the execution of eye movements, do not project to area V4.

\subsubsection{Optogenetic targeting of the projection from FEF to area MT}

The aim of the main study of this thesis was to inhibit the projection from the FEF to area MT by using optogenetics. Optogenetics (Boyden et al., 2005) has been proven to be a reliable method to modulate neural activity in rodents and has sparsely also been used in non-human primates (e.g., Diester et al., 2011; Galvan et al., 2017). Optogenetic stimulation can increase or decrease neural activity by using excitatory or inhibitory opsins (e.g., Han et al., 2009, 
2011). It expands electrophysiological studies with tools that have not been available before (for a review see Tye and Deisseroth, 2012).

One of the new possibilities optogenetics provides is cell-type specific activation or inactivation of neurons without affecting other cell types. In rodents, this is a well-tried method since in addition to the use of viral vectors, transgenic animals have been developed for optogenetic experiments (for a review see Zeng and Madisen, 2012; Ting and Feng, 2013). In non-human primates, however, the situation is different due to the lack of transgenic animals. Research in non-human primates is dependent on using viral vectors. These are limited in their packaging capacity, and, therefore, only a handful of promoters is available. However, recently an approach of combining two viral constructs, which allowed the use of the Cre/lox system, has been successful in targeting dopaminergic neurons in non-human primates (Stauffer et al., 2016).

A second new possibility is that optogenetics allows to specifically target the axonal projections between two brain areas. This previously impossible method allows to do experiments in a much more controlled and precise way, because the interaction of two areas can be directly assessed. Opsins have been shown to be reliably integrated into the membrane of axons, and often, viral constructs are designed in a way that enhances trafficking within the neuron (e.g., Gradinaru et al., 2010). In rodents, targeting projections is already a standard method. Several studies showed effects of projection stimulation on behavior (e.g., Tye et al., 2011; Burgos-Robles et al., 2017). However, the application of optogenetics to the non-human primate is slow, so that only two studies stimulated projections optogenetically. Inoue and colleagues successfully targeted the projection from the FEF to the superior colliculus with an excitatory opsin (Inoue et al., 2015). They showed that laser stimulation of the projection reliably evoked saccades. Galvan et al. (2016) injected a viral vector into the motor cortex and stimulated the projections in the motor thalamus. Optogenetic excitation of the axon terminals significantly changed activity of neurons in the motor thalamus. Although in rodents it has been done successfully, no study in the non-human primate, so far, inhibited the projection between two brain areas.

The FEF has been targeted in several optogenetic studies in the non-human primate (Han et al., 2009, 2011; Gerits et al., 2012; Ohayon et al., 2013; Acker 
et al., 2016). All of these studies reported a change in neural activity induced by optical stimulation. However, only Gerits et al. (2012) and Acker et al. (2016) found a change in the behavioral performance of the monkeys. In the study conducted by Gerits and colleagues, optogenetic stimulation changed the latency of evoked saccades, but not the accuracy or end point of saccadic eye movements. Acker and colleagues found that optogenetic inhibition of the FEF resulted in a large increase in the error rate of the monkeys and an increase in the scatter of saccade end-points.

Taken together, all these results indicate that the FEF and the axonal projection from the FEF to area MT can be targeted and inhibited by optogenetics. 


\subsection{Part II}

\subsubsection{The attentional blink}

The attentional blink is a perceptual phenomenon that depicts limitations of top-down attention in humans, that is, attention has a limited temporal resolution. It was originally named by Raymond et al. (1992), who tested in several experiments how processing of a visual target stimulus (T1) affects the processing of a subsequently shown target stimulus (T2). These two target stimuli were presented within a stimulus stream by rapid serial visual presentation. The time between the two target stimuli (target onset asynchrony, TOA) was systematically varied, and two behavioral conditions were compared: a single-task in which subjects only had to detect T2, and a dual-task in which subjects had to identify $\mathrm{T} 1$ and to detect $\mathrm{T} 2$. Both behavioral conditions did not differ in their sensory information, but in the task instructions the subjects received. In this way, the authors compared the performance of the subjects to detect T2 in the stimulus stream, either with or without prior T1 identification. This comparison allowed to judge about the impact of $\mathrm{T} 1$ processing on $\mathrm{T} 2$ processing. The authors found that when $\mathrm{T} 2$ was presented within $450 \mathrm{~ms}$ after $\mathrm{T} 1$, subjects showed a deficit in detection of $\mathrm{T} 2$ in the dual-task, and these results indicated that T1 processing interfered transiently with the processing of $\mathrm{T} 2$.

Since the initial report of the phenomenon a tremendous number of studies have dealt with this finding and have shown that it occurs for different stimulus types, such as faces (Marois et al., 2004), visual scenes (Livesey and Harris, 2011), orientation (Joseph et al., 1997), or color (Ross and Jolicoeur, 1999). Most of the tested stimuli are processed by the ventral visual pathway.

The attentional blink exhibits some characteristics that are important to understand its underlying mechanisms. One of the prominent features is the so-called 'lag1-sparing": in around half of the attentional blink experiments, detection of T2 is not impaired when it is presented directly after T1 (Visser et al., 1999). In addition, several target stimuli can be detected without impairment in case no intervening distractor stimulus is shown (Di Lollo et al., 2005; Olivers et al., 2007). Masking of the two target stimuli has been shown to 
be an important factor in causing an attentional blink (Raymond et al., 1992; Chun and Potter, 1995; Grandison et al., 1997; Visser and Ohan, 2007). These results indicate that there is not a general inability to process several stimuli presented in close temporal proximity, but it rather suggests the existence of a mechanism that protects target processing from distracting information. In addition, the processing of $\mathrm{T} 2$ is affected at a late stage of processing, probably comprising the consolidation into working memory or the preparation of the task response, whereas early processing stages do not seem to be severely influenced (Luck et al., 1996, Sergent et al., 2005).

Several studies showed that features of two visual stimuli can be falsely bound together (as 'illusionary conjunctions', Treisman and Gelade, 1980) when they are presented in close temporal proximity (e.g., Lawrence, 1971; McLean et al., 1983; Botella and Eriksen, 1992; Raymond et al., 1992). This type of error could be prevented or diminished with a temporal attentional filter that only temporarily influences the gain of neural activity whenever a target stimulus is presented. Such kind of a filter has been proposed by Aston-Jones and Cohen (2005) as a role for the phasic activity of the locus coeruleus (LC) in the brain stem.

However, despite the reliable occurrence of the deficit per se, the underlying neural circuits of the attentional blink are still not clarified. Several theoretical models have been developed trying to explain the underlying mechanisms (for a review see Dux and Marois, 2010).

One of the models explaining the attentional blink assumes a role of the locus coeruleus in causing the attentional blink (Nieuwenhuis et al., 2005). The LC has been shown to be involved in visual target detection (Aston-Jones et al., 1994). It shows periods of phasic response which are followed by a refractory period in which the activity of the LC is inhibited (Foote et al., 1980; Aston-Jones and Bloom, 1981a). As mentioned above, the phasic activity has been suggested to act as a temporal attentional filter (Aston-Jones and Cohen, 2005), and, since the time course of the LC's refractory period is similar to the duration of the attentional blink, the LC has been suggested to play a role in evoking an attentional blink (Nieuwenhuis et al., 2005). 


\subsubsection{The locus coeruleus}

The LC is a part of the brain stem that projects to almost all areas of the brain and provides the main source of noradrenaline (Freedman et al., 1975; Garver and Sladek, 1975; Berridge and Waterhouse, 2003). It receives a major input from the prefrontal cortex (Arnsten and Goldman-Rakic, 1984; Jodo et al., 1998), but also from structures like the nucleus prepositus hypoglossus, which is directly involved in the control of eye movements (Aston-Jones et al., 1986). The LC plays a role in controlling the sleep-waking cycle (e.g., Aston-Jones and Bloom, 1981b).

During the waking state, the LC operates in two modes, one is characterized by a tonic firing of its neurons and is thought to promote non-specific aspects of behavior like arousal and responsiveness (Usher, 1999). The other mode is characterized by a phasic activity followed by a refractory period lasting several hundred milliseconds, and occurs in response to salient sensory stimuli (Foote et al., 1980; Aston-Jones and Bloom, 1981a). However, the phasic response can also be very specific. For example, Aston-Jones et al. (1994) recorded neural activity in the LC of monkeys while they were performing a visual discrimination task. They found that target detection of a visual stimulus was accompanied by a phasic response of the LC, whereas distractor presentation did not evoke such a response. Moreover, as a subsequent study showed, the latency of this phasic response can be dependent on task difficulty. Therefore, rather than being a pure sensory response, the phasic activity can reflect the behavioral relevance of a target stimulus (Rajkowski, 2004).

In addition to its role in sleep and awake states, several studies suggest that the locus coeruleus has a direct effect on pupil size (Gilzenrat et al., 2010; Murphy et al., 2014; Joshi et al., 2016).

\subsubsection{The effect of attention on pupil size}

The size of the pupil is changed dependent on the light intensity that falls onto the retina and is adjusted to reach an optimum of sensitivity and visual acuity (Campbell and Gregory, 1960). However, the size of the pupil is not only driven reflexively by external light. It has been known for a long time that higher cognitive processes have an impact on the size of the pupil (e.g., 
Loewenstein, 1920; Hess and Polt, 1964; Beatty, 1982). In recent years, as elucidated in the following, pupil size has more and more been used as a noninvasive, physiological measure of top-down attentional processes.

Detection of a visual target stimulus is associated with a dilation of the pupil (Privitera et al., 2010). In addition, pupil size can scale with the number of attended objects (Alnaes et al., 2014; Wahn et al., 2016). It can reflect the presentation frequency of a visual stimulus and this reflection can be modulated in size by visual attention (Naber et al., 2013).

Binda et al. (2013) and Mathot et al. (2013; 2014) showed that the pupillary response can reflect the focus of attention: Attending to a bright stimulus evoked a different pupil response than attending to a dark stimulus, and, hence, reflected the focus of spatial attention. In addition, the focus of featurebased attention can be apparent in the pupil size (Binda et al., 2014).

These results are in agreement with studies showing that optical illusions and expectations about a visual scene can influence the pupil diameter in isoluminant presentations that only vary in high-level contents of visual information (Laeng and Endestad, 2012; Naber et al., 2013). Images of the sun, for example, evoked different changes in pupil size dependent on the orientation of an image. Inverted images of the sun reduced the pupil constriction usually found for upright images of the sun (Naber et al., 2013).

Hoeks and Levelt (1993) developed a method with which the measure of pupil size over time during a behavioral task can be used to reconstruct the underlying attentional processes that induced changes of pupil size. This method has been proven to be a reliable model even under high-frequency stimulus presentation (Wierda et al., 2012). Wierda and colleagues applied the model to the analysis of pupil size during an attentional blink task. They found that trials in which T2 was missed in the dual-task showed a different pattern of underlying attentional pulses than trials in which T2 was correctly reported. The pulses evoked by $\mathrm{T} 1$ presentation were higher and the pulses underlying T2 presentation were lower in amplitude in trials in which T2 was missed. In the same year, also Zylberberg et al. (2012) measured pupil dilation in an attentional blink task. They reported that during the time the attentional blink typically occurs, pupil amplitude was smaller and pupil dilation evoked by T2 
presentation was delayed. These results indicate that pupil size can help to interpret the results of attentional blink and other attentional experiments. However, the underlying neural pathways that control pupil size during cognitive tasks are not well understood, and, therefore, the interpretation of the results is difficult.

The size of the pupil is regulated by an interplay of two eye muscles in the iris: the musculus sphinter pupillae is responsible for the contraction of the pupil, while the musculus dilatator pupillae mediates the dilation of the pupil (for a review see Loewenfeld, 1999). These muscles are controlled by a network of structures of the peripheral nervous system (Loewenfeld, 1999).

However, it has been shown that microstimulation of subcortical structures, like the superior colliculus and the locus coeruleus, changes the size of the pupil of non-human primates (Wang et al., 2012; Joshi et al., 2016). Furthermore, a recent study found that microstimulation of a cortical structure, i.e., the FEF, changes pupil size (Lehmann and Corneil, 2016). In accordance with the behavioral studies mentioned above, these results indicate that subcortical and cortical areas that control higher cognitive functions, also have a direct effect on pupil size during cognitive tasks. However, the evidence of the underlying neural mechanisms is still sparse as investigations just started in recent years. Currently available evidence suggests that the locus coeruleus has the most direct effect on pupil size (Gilzenrat et al., 2010; Murphy et al., 2014; Joshi et al., 2016). 



\section{Chapter 2 \\ Project manuscripts}

1. An anatomical viability evaluation of using optogenetics to examine the fronto-visual and fronto-parietal network in the macaque

2. Optogenetic inhibition of FEF input to area MT reduces attentional modulation of neuronal responses

3. An attentional blink with motion stimuli and in a task combining motion and letter stimuli

4. Pupil size suggests that the locus coeruleus is involved during attentional blink tasks without a direct perceptual consequence 



\section{An anatomical viability evaluation of using op- togenetics to examine the fronto - visual and fronto - parietal network in the macaque}

Scientists that contributed to this project in alphabetical order: Michal G. Fortuna $^{1}$, Alexander Gail ${ }^{1}$, Jens Gruber ${ }^{3}$, Hao Guo ${ }^{1}$, Janina Hüer $^{1}$, Hansjörg Scherberger ${ }^{2}$, Lara Timantra Schiller ${ }^{3}$, Jochen Staiger $^{4}$ and Stefan Treue ${ }^{1}$

\section{Affiliations}

1 Cognitive Neuroscience Laboratory, Deutsches Primatenzentrum GmbH, Göttingen, Germany

2 Neurobiology Laboratory, Deutsches Primatenzentrum GmbH, Göttingen, Germany

3 Medical RNA Biology, Deutsches Primatenzentrum GmbH, Göttingen, Germany

4 Department of Neuroanatomy, Georg-August University, Göttingen, Germany

Author contributions:

M. G. Fortuna: Design of experiment, viral vector injection, perfusion and extraction of brain, cutting of brain, majority of immunohistochemistry and imaging, histological analysis, contribution to manuscript's method section

A. Gail: Design of experiment, viral vector injection, histological analysis

J. Gruber: Viral vector development, testing and handling, implementation and compliance of genetic engineering safety regulations

H. Guo: Design of experiment, viral vector injection

J. Hüer: Design of experiment, idea to target projections with optogenetics, choice of viral vectors, viral vector injection, cutting of brain, minority of immunohistochemistry and imaging, implementation and compliance of genetic engineering safety regulations, animal handling and monitoring, histological analysis, manuscript writing 
H. Scherberger: Design of experiment, viral vector injection, implementation and compliance of genetic engineering safety regulations, histological analysis L. T. Schiller: Viral vector development, testing and handling

J. Staiger: Viral vector testing, infrastructure and expertise for histological analysis

S. Treue: Design of experiment, viral vector injection, histological analysis

Figure 2.1 - J. Hüer

Figure 2.2 - Staining, imaging: M. G. Fortuna; processing, labeling: J. Hüer Figure 2.3 - Staining, imaging: M. G. Fortuna; processing, labeling: J. Hüer Figure 2.4 - Staining, imaging, processing: M. G. Fortuna; labeling: J. Hüer Figure 2.5 - Staining, imaging: M. G. Fortuna; processing, labeling: J. Hüer Figure 2.6 - Staining: M. G. Fortuna; imaging: M. G. Fortuna and J. Hüer; processing, labeling: J. Hüer

Figure 2.7 - Staining, imaging, processing: M. G. Fortuna; Labeling: J. Hüer Acknowledgements

We thank Karl Deisseroth for viral constructs and Michael Feyerabend for helping with the testing of the viral constructs.

\section{Introduction}

Optogenetic manipulation of neural activity has been increasingly utilized in non-human primates in recent years. One of the most promising potentials it offers to systems neuroscience is the possibility to selectively stimulate axonal projections between two brain areas and investigate causal interactions in a direct way that has not been possible before.

Successful optogenetic experiments depend on choosing a combination of virus/ viral serotype, promoter and opsin that results in decent expression of opsins in an area of interest. However, the optimal viral construct varies between species, brain area and experimental needs. Experiments in non-human primates cannot easily be repeated and individuals are usually highly valuable, since they are often trained for periods of months or even years. In addition, experiments usually last weeks until months and therefore high and stable 
opsin expression would be desired, which at the same time is not causing neuronal cell death.

We have evaluated the viability of using optogenetics to study the frontoparietal as well as fronto-visual cortical network with a specific focus on the axonal connections within these networks. We focused our analysis on the connections of frontal area PMv and posterior parietal area MIP (e.g., Bakola et al., 2017), and on the connections of prefrontal area FEF and visual area MT (Ninomiya et al., 2012; Stanton et al., 1995; Schall et al., 1995).

Several requirements should be fulfilled to successfully use optogenetics to manipulate axonal projections. Projection targeting necessitates a decent spatial spread of transduction around the injection location to cover major parts of an area of interest. Long-range projections can have a length of up to several centimeters (Markov et al., 2013), and they can branch to target different areas (Rockland et al., 2013). Therefore, the total surface area of the cell membrane can be large, and the transport ways within the cell long. Hence, it can take a while until a sufficient density of opsins is reached at the axonal ends in a distant projection area. The density of the opsins must be high enough so that optical stimulation causes sufficient de- or hyperpolarization of axons to either evoke action potentials or inhibit incoming spikes. The critical point here is to conduct the stimulation experiments during a time of sufficiently high and stable opsin expression. Since this information is not accessible during ongoing experiments with behaving non-human primates, any information about the development of opsin expression over time is highly valuable.

Ideally, projection targeting necessitates a viral vector that only enters the local somata and dendrites at the injection location. Axons in a given area stem from a variety of brain areas, and uptake of the viral vector by the axons can result in retrograde transport of the vector to cell bodies in distant locations (Castle et al., 2014). Depending on the amount of retrograde transport, it can be a confounding factor when one assumes to only stimulate projecting axons, but instead the stimulation also affects cell bodies of retrogradely transduced neurons.

We chose rAAV2/5 as a serotype for our experiment. In direct comparison with other serotypes, rAAV2/5 showed reliably high transduction efficiency (Taymans et al., 2007; Burger et al., 2004; Markakis et al., 2010; Watakabe 
et al., 2015). However, like all other AAV-serotypes except AAV2/2, retrograde transport has been reported in several studies (Burger et al., 2004; Aschauer et al., 2013). AAV2/2 might be the cleanest viral vector for projection targeting and it has been successfully used in primates for that purpose (Inoue et al., 2015). However, it appeared to be the least effective serotype throughout the above-mentioned comparison studies, and can show less anterograde transport of the opsins along the axons compared to other serotypes (McFarland et al., 2009).

AAV5 binds to the platelet-derived growth factor alpha-receptor (PDGFR $\alpha$, Di Pasquale et al., 2003) and 2,3-linked sialic acid (Kaludov et al., 2001; Walters et al., 2001), which both can be found in the membrane of neurons throughout the brain (Vignais et al., 1995; Oumesmar et al., 1997; Schnaar et al., 2014). Which part of the neuron takes up the viral vector depends on the presence of these factors within the membrane. It is unknown, however, how PDGFR $\alpha$ and 2,3-linked sialic acid are distributed within the membrane of neurons in our areas of interest.

Long-range projections originating from cortical areas are generally assumed to be excitatory (e.g., DeFelipe and Fariñas, 1992; Anderson et al., 2011), but, to a small extent, there are also inhibitory long-range projections (Lee et al., 2014; Rock et al., 2016). Optogenetics, in theory, offers the possibility to target different cell types by using specific promoters. However, all but one of the currently available promoters that can be used in combination with a viral vector are relatively unspecific in their targeting. The most specific one is the $\alpha$ CaMKII-promoter, which is used by excitatory cells, but not GABAergic cells (Benson et al., 1991, 1992; Jones et al., 1994; Liu and Jones, 1996; Tighilet et al., 1998; Han et al., 2009). However, not all of the excitatory cells seem to use it (Jones et al., 1994; Liu and Jones, 1996; Tighilet et al., 1998). Therefore, it is unclear in how far long-range projection neurons use the $\alpha$ CaMKII-promoter. The $\alpha$ CamKII-promoter has only been used in one study in the primate to target projections from the cortex to the thalamus (Galvan et al., 2016). Since we were interested in targeting projections in the most specific way, we examined whether the $\alpha$ CaMKII-promoter can be used to target long-range projection neurons in our areas of interest.

We injected three different viral vectors (rAAV2/5- $\alpha$ CaMKII-hChR(H134R)- 
eYFP, rAAV2/5- $\alpha$ CaMKII-eNpHR3.0-mCherry, rAAV2/5- $\alpha$ CaMKII-eNpHR 3.0-eYFP) into four distinct cortical areas: area FEF, area PMd, area PMv and V1. We analyzed expression spread in the injection area, expression in axonal projections and the extent of retrograde transport.

\section{Methods}

\section{Animal}

All animal work and housing was conducted in accordance with all applicable German and European regulations. The scientists in this study are aware and are committed to the great respnsibility they have in ensuring the best possible science with the least possible harm to any animals used in scientific research. All animal procedures have been approved by the responsible regional government office (Niedersächsisches Landesamt fur Verbraucherschutz und Lebensmittelsicherheit [LAVES], Oldenburg, Germany) under the permit number 3392 42502-04-13/1100. All surgical and imaging procedures were done under appropriate anesthesia, with appropriate analgesics and in accordance with German laws governing animal use.

All experiments reported herein were performed on one male rhesus macaque (Macaca mulatta, age: 10 years, weight: $10 \mathrm{~kg}$ ). The animal was group-housed with other macaque monkeys in a facility of the German Primate Center in Göttingen (Germany). The animal had unrestricted access to food and water and was provided with an enriched environment. The animal was taken care of by the animal caretakers, technical assistants and scientists, and monitored on a daily basis by the veterinarians of the German Primate Center.

\section{Virus injection}

Viral vectors were injected under anesthesia. We determined the stereotactic coordinates of the injection areas with the help of a prior anatomical MRI scan. Two craniotomies were done, one in the frontal left hemisphere providing access to areas FEF, PMv and PMd, the other in the right hemisphere providing access to area V1. We opened the dura and determined the exact injection locations based on anatomical landmarks. We injected three different viral vectors: (a) rAAV2/5- $\alpha$ CaMKII-hChR2(H134R)-eYFP- 


\begin{tabular}{ccccc}
\hline area & construct & vol & count & depths \\
\hline \hline & & & & \\
FEF left & rAAV2/5- $\alpha$ CaMKII-eNpHR3.0-mCherry & $7 \mu l$ & 1 & 7 \\
PMv left & rAAV2/5- $\alpha$ CaMKII-hChR2(H134R)-eYFP & $9 \mu l$ & 3 & 3 \\
PMd left & rAAV2/5- $\alpha$ CaMKII-eNpHR3.0-eYFP & $4.5 \mu l$ & 1 & 3 \\
V1 right & rAAV2/5- $\alpha$ CaMKII-eNpHR3.0-mCherry & $9 \mu l$ & 3 & 3 \\
& & & & \\
\hline
\end{tabular}

Table 2.1: Injection parameters
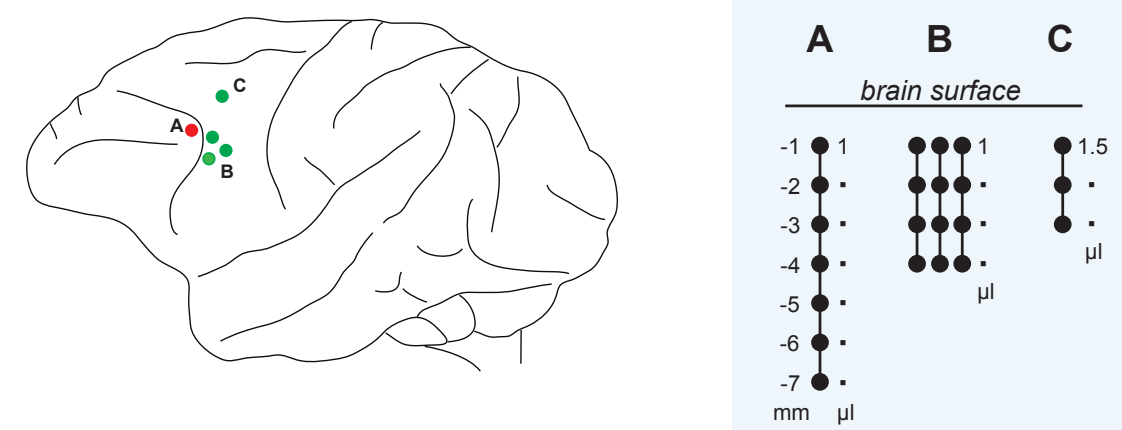

Figure 2.1: Injection sides in the left hemisphere. We injected in three different frontal areas: (A) FEF, (B) PMv, and (C) PMd. The number of depths, penetrations and the injected volume is shown for each of the three brain areas.

WPRE (titer: $8.5 \times 10^{12} \mathrm{vg} / \mathrm{ml}$ ), (b) rAAV2/5- $\alpha$ CaMKII-eNpHR3.0-mCherryWPRE (UNC Vector Core, titer: $4.7 \times 10^{12} \mathrm{vg} / \mathrm{ml}$ ), and (c) rAAV2/5- $\alpha$ CaMKIIeNpHR3.0-eYFP-WPRE (produced in-house, titer: $7.8 \times 10^{8} \mathrm{vg} / \mathrm{ml}$ ). Construct (a) was injected into left PMv, construct (b) into left FEF and right V1 and construct (c) into left PMd (table 2.1).

Each viral vector solution was loaded into a gas-sterilized syringe (from Göhler HPLC-Analysentechnik or CS-Chromatographie-Service GmbH; volume: 10 $\mu 1$ or 25ul, needle diameter: 32 gauge, sharp tip) under sterile conditions. The syringe was fixed to a manual injector, which was attached via a one-axis coarse manipulator (BE-8, Narishige) to a stereotaxic frame.

In each of the four brain areas, we injected at one or three locations, and at each location, we injected at several depths (for details see table 2.1 and figure 2.1). We started at the deepest point and injected $1 \mu \mathrm{l}$ at each depth $(1.5 \mu \mathrm{l}$ 
in area $\mathrm{PMd}$ ) with a speed of $200 \mathrm{nl} / \mathrm{min}$. We waited 5 minutes after each injected microliter and before retracting the needle by $1 \mathrm{~mm}$ to the next depth or before moving to the next injection location. In V1 and PMv, we injected at three locations spanning a triangle with a side length of $1 \mathrm{~mm}$ to $1.5 \mathrm{~mm}$. In FEF and PMd, we only injected at one location.

The dura was sutured after the injections were completed. It was covered with the previously removed bone and fixed to the surrounding skull.

The animal was left back into his group after several days of recovery.

\section{Removal of the brain for histological analysis}

After a survival period of 10 weeks, we conducted acute electrophysiological recordings under anesthesia. The results are not part of this report. At the end of the acute recordings, the animal was very deeply anesthesized with pentobarbital. The animal was perfused transcardially with heparinized PBS until the blood cleared, followed by 31 of $4 \%$ paraformaldehyde in PBS (phosphate buffer saline, 0.1M, pH 7.6). After the perfusion, the brain was removed from the skull and stored in the perfusion fixative at $4^{\circ} \mathrm{C}$.

\section{Histology}

The fixed brain was cut into blocks containing the areas of interest. The blocks were cut into 50um thick (semi)coronal sections on a vibrating microtome. The sections were kept in a cryoprotective solution (20\% glycerol + $30 \%$ ethylene glycol in $0.05 \mathrm{M}$ phosphate buffer, $\mathrm{pH} 7.4$ ) at $-20^{\circ} \mathrm{C}$ until further processing. All histological stainings were performed on free-floating sections, which for each procedure were washed once with PB (phosphate buffer, 0.1M, $\mathrm{pH} 7.4$ ), followed by three washings in TBS (0.1M, tris-buffered saline, $\mathrm{pH}$ 7.6). They were blocked with $10 \%$ normal serum (donkey + horse, 1:1) in TBS with $0.2 \%$ Triton-X (TBS-T) for 1 h at RT and incubated with a primary antibody overnight in $2 \%$ normal serum (donkey + horse, 1:1) in TBS-T at $4^{\circ} \mathrm{C}$. Subsequently, the sections were washed three times with TBS and incubated with a secondary antibody for $1-2 \mathrm{~h}$ at RT in TBS-T with $2 \%$ serum. After washing three times with TBS and three times with PB, the sections were mounted on glass slides, air-dried and coverslipped with a mounting medium (Fluoroshield Mounting Medium, Abcam). All serums were ordered 
via Dianova from Jackson ImmunoResearch Laboratories, Inc. and all used antibodies can be found in the supplementary material (table S1, S2). Images where taken by an Axio Imager M2 microscope (Zeiss) controlled by and outlined with Neurolucida (MBF Bioscience). Images were further processed with ImageJ.

\section{Results}

\section{Transduction efficiency}

We wanted to compare the transduction efficiency of two different viral constructs. Therefore, we used the same injection parameters (volume, number of locations, injection speed) for areas PMv and V1, but injected two different viral constructs. Our aim was to compare the spatial spread of opsin expression in these two brain areas as one measure for transduction efficiency. In the scope of this thesis, the transduction efficiency was only analyzed for area PMv. In addition, we analyzed the percentage of neurons expressing the opsin in area FEF.

Figure 2.2 shows the expression spread around two of the three injection tracks in area PMv. The spread of neurons expressing the opsin was estimated to be $5.1 \mathrm{~mm}$ and $5.7 \mathrm{~mm}$ in the longitudinal direction of the two tracks. The lateral spread was around $3.5 \mathrm{~mm}$. We estimated the area of opsin expression with the help of ImageJ as $14.4 \mathrm{~mm}^{2}$. Dividing the area by the number of injection locations along the two tracks (i.e. 6), and considering a circular distribution, results in an expression radius of $0.88 \mathrm{~mm}$ around each injection location.

In area FEF, we stained some of the brain sections for mCherry and NeuN (figure 2.3), and determinend the percentage of neurons that expressed the opsin eNpHR3.0. We estimated that around $47 \%$ of neurons in the vicinity of the injection trask expressed the opsin (table 2.2).

As mentioned above, we did not yet analyze the injection in area V1. In addition, we were not able to retrieve the injection side in area PMd. Therefore, we assume that the viral construct injected in area PMd was not working as expected. 


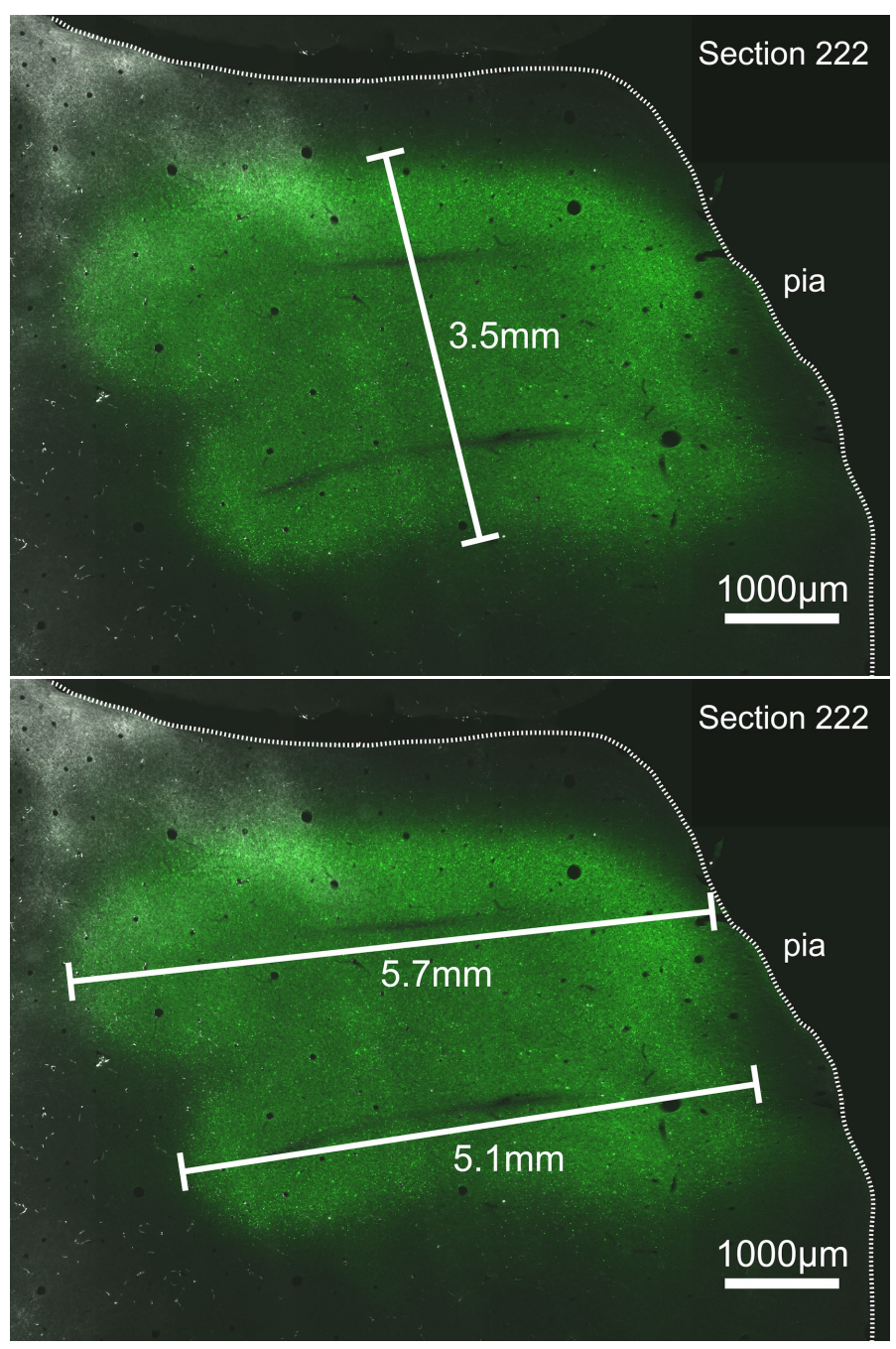

Figure 2.2: Injection tracks in area PMv. We estimated the longitudinal and lateral spread of eYFP-positive neurons (green) along the injection tracks. 


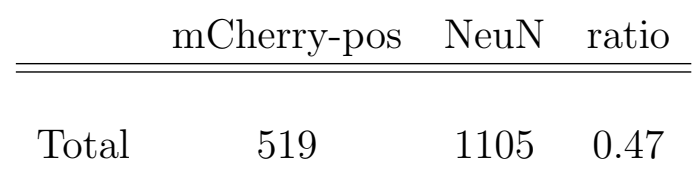

Table 2.2: Cell counts of mCherry-positive neurons in area FEF. We counted the percentage of cells around the injection track based on section 173 .

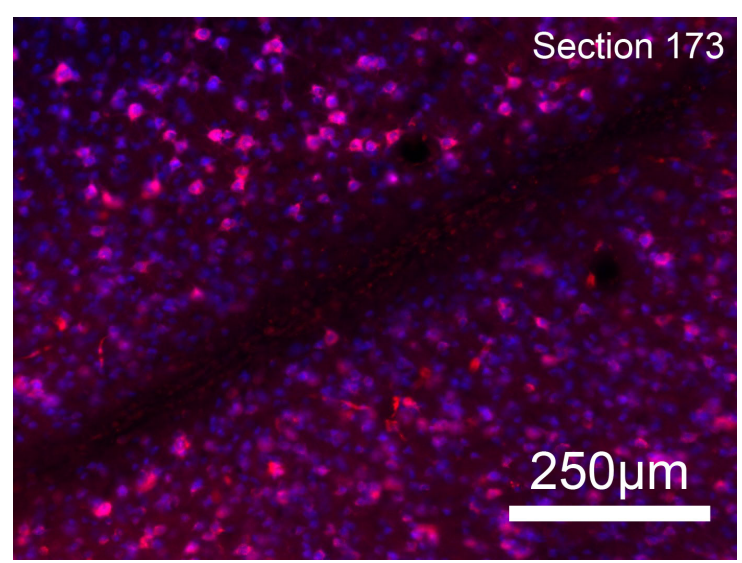

Figure 2.3: Injection track in area FEF. Neurons stained for NeuN are shown in blue, mCherry-positive neurons in red.

\section{Opsin expression in axons}

We wanted to examine whether the $\alpha$ CaMKII-promoter can be used to target axonal projections in our networks of interest. In addition, we were interested in whether a period of 10 weeks is sufficient to observe a decent opsin expression within the axon terminals in areas distant from the injection region.

We observed substantial opsin expression in all parts of the neurons. Figures 2.4 and 2.5 show that opsins could be found in the cell bodies as well as dendrites and axons of transduced neurons. We found high opsin expression in axons in the injection locations (figure $2.4 \mathrm{~A}$ ), axons leaving the injection area (figure 2.5) and neighboring areas (figure $2.4 \mathrm{~B}$ ).

Moreover, we found axons positive for mCherry or eYFP in brain areas distant from the injection locations. Figure 2.6 shows long-range projections from area FEF in area MT. Axons expressing the opsin were mainly found in infragranular layers and layer I in area MT. A similar pattern was found in the parietal area MIP (figure 2.7). Projecting axons from are PMv were mainly located in infragranular layers, and to a lesser extent in layer I. 

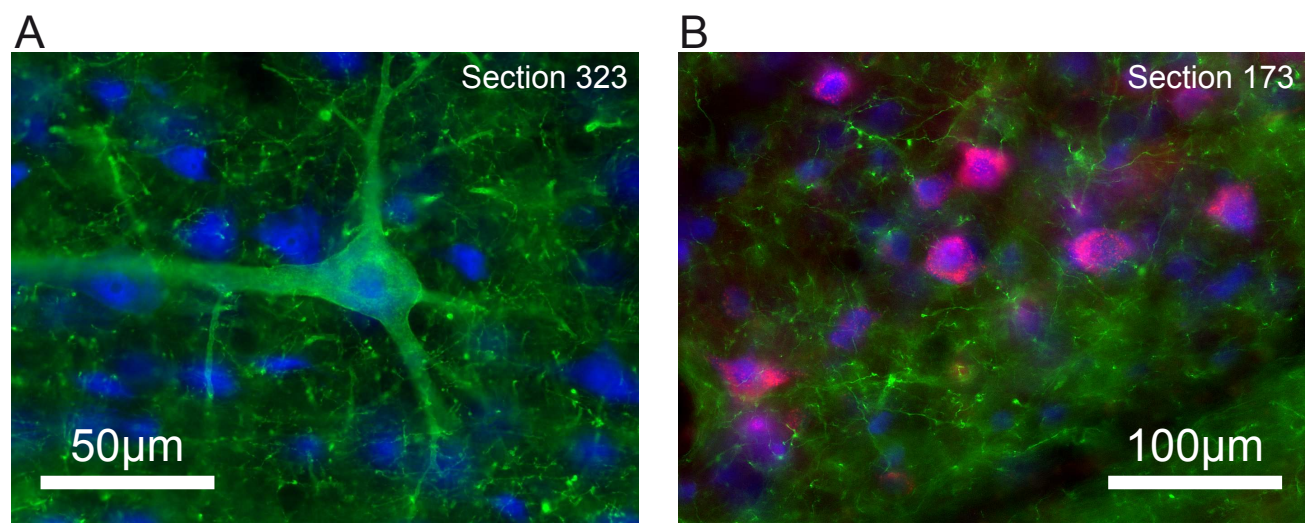

Figure 2.4: (A) Opsin expression in area PMv, (B) opsin expression in axons in area FEF.

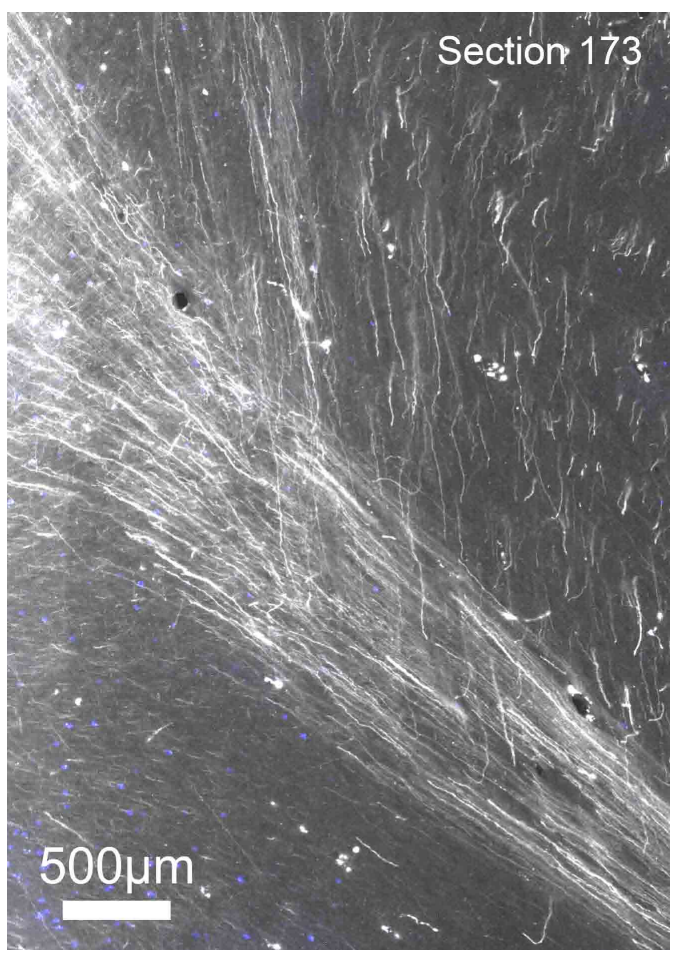

Figure 2.5: Opsin expression in axons leaving the injection area FEF (mCherrypositive axons are shown in white). 


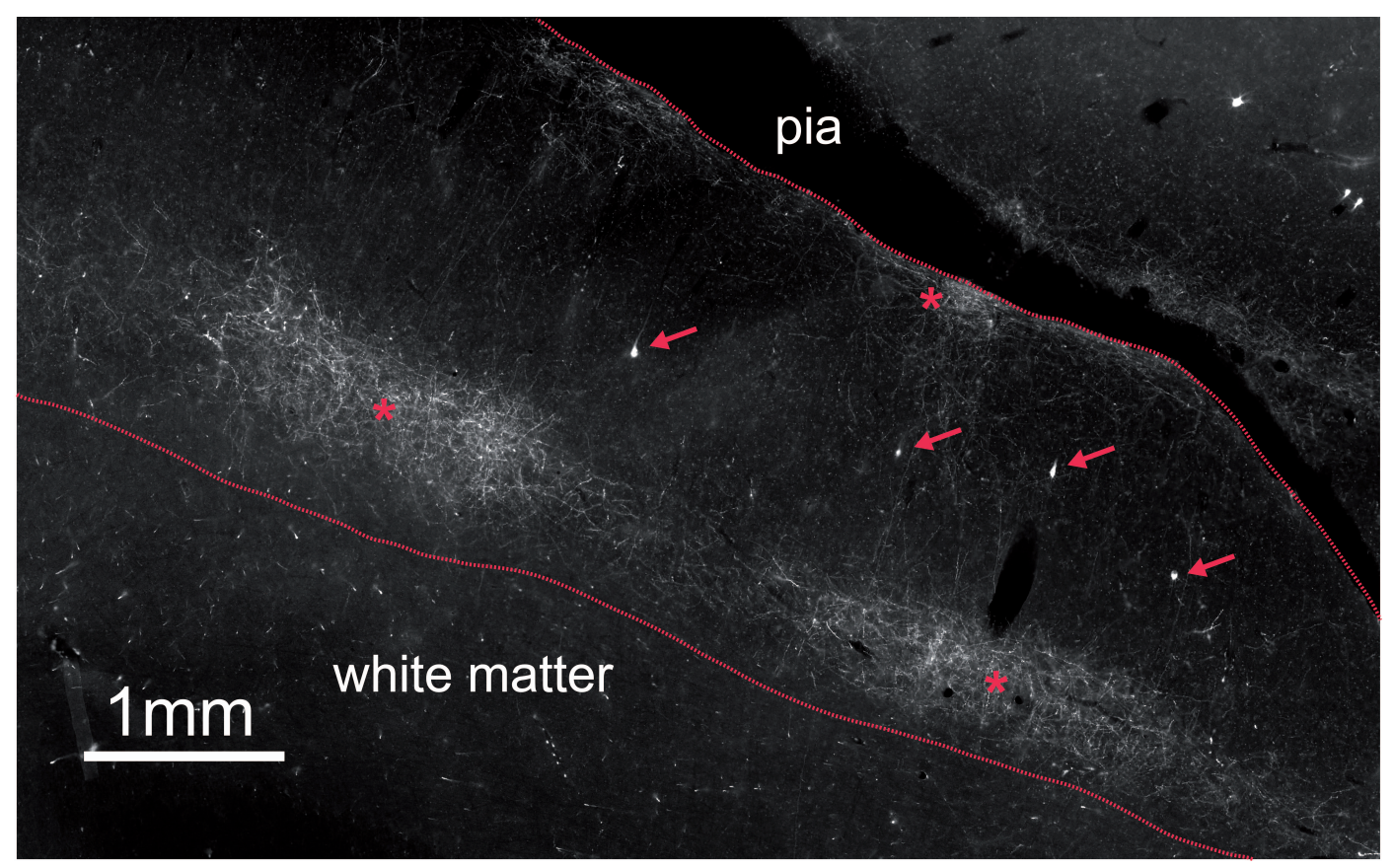

Figure 2.6: Opsin expression in FEF axons projecting to area MT. Red arrows show retrogradely labeled cells, red asterisks mark mCherry-positive FEF axons (white). Projections from FEF were mainly found in infragranular layers and layer 1 of area MT. 


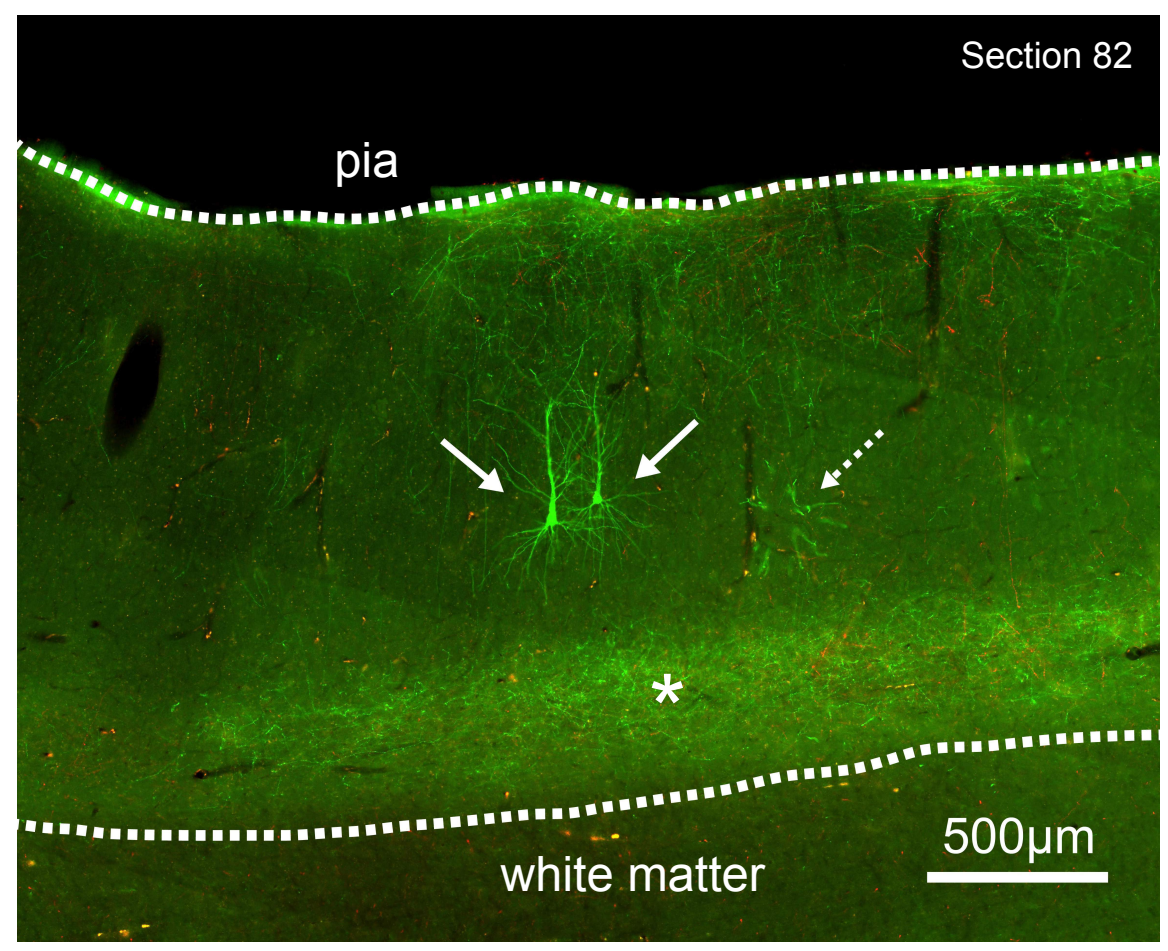

Figure 2.7: Opsin expression in PMv axons projecting to area MIP. White arrows show retrogradely transduced cells. The white asterisk marks eYFP-positive PMv axons (green), which were mainly located in infragranular layers of area MIP. 


\section{Retrogradely transduced neurons}

We examined the amount of retrograde transport of the viral vectors in our areas of interest. We found a small number of retrogradely transduced cells in several brain areas distant from the injection area. Figure 2.6 shows retrogradely transduced cells in visual area MT. They were stained for mCherry and must have taken up the viral vector in area FEF. These neurons were mainly located in supragranular layers in area MT.

We found retrogradely labeled cells also for the viral vector that has been injected in area PMv. Figure 2.7 shows at least two retrogradely labeled cells (marked by the bold arrows; a third potentially transduced cell is marked by the dashed arrow) in supragranular layers of parietal area MIP.

\section{Discussion}

We explored histologically if the fronto-visual and fronto-parietal network and its long-rang projections can be targeted with optogenetics in the non-human primate. In the scope of this thesis, we analyzed the results of injecting one viral vector into the $\mathrm{FEF}$ and a different viral vector into $\mathrm{PMv}$ of the left hemisphere of one monkey.

We found that injection in both areas resulted in decent opsin expression around the injection tracks. In area PMv, injection of $1 \mu$ l of viral vector solution transduced neurons within a radius of approximately $0.9 \mathrm{~mm}$ around an injection point. This radius is similar to what other studies reported. Han and colleagues, for example, found that after injection of $1 \mu l$ of viral vector neurons were transduced within a radius of $0.7 \mathrm{~mm}$ around the injection location (Han et al., 2009).

In area FEF, we found that around $47 \%$ of neurons expressed the opsin along part of the injection track. This value is in the same range as values reported by previous studies in the non-human primate. For example, Diester et al. found that up to $44 \%$ of neurons expressed opsins after injection of viral vectors and using pan-neuronal promoters (Diester et al., 2011).

Our results show that the $\alpha$ CamKII-promoter is used by projection neurons in areas FEF and PMv. Even though it is known that the $\alpha$ CamKII-promoter is used by excitatory neurons (e.g., Benson et al., 1991, 1992; Jones et al., 1994) 
and that most projection neurons are excitatory (DeFelipe and Fariñas, 1992; Anderson et al., 2011), to our knowledge it has not been systematically investigated whether projection neurons use the $\alpha$ CamKII-promoter. We found high opsin expression in all parts of the neurons, that is, in local neurons in the injection area, in axons in the white matter, and in axons in distant projection areas. FEF and PMv neurons projecting to areas MT and MIP showed high opsin presence in the axonal terminals in those target areas. This finding confirms that opsins are incorporated in the membrane of long-distance projections in our areas of interest, and, consequently, those projections can be stimulated optogenetically.

It further indicates that opsin expression is already high in all parts of the neurons after a waiting period of 10 weeks after virus injection, and stimulation experiments could be conducted. However, we cannot draw any conclusion about the stability of the expression. Since the density of the opsins can influence the results of stimulation, a change of expression over time can also change the results of stimulation over time. This is an important factor and should be examined in future studies, especially because non-human primates in neurophysiology often participate in a number of consecutive experiments. We found cells that must have taken up the viral construct retrogradely. Previous studies reported to only find a very small number of retrogradely labeled cells. The number of cells in our study is higher than what has previously been reported (e.g., Diester et al., 2011). However, we do not consider it as a major factor impeding optogenetic stimulations, though, it should be kept in mind for any stimulation experiment.

In addition to evaluating the viability of using optogenetics to target the fronto-visual and fronto-parietal network, we can confirm some of the previous reports about the localization of projecting neurons and projection terminals in the areas of these networks. We found that projections from FEF neurons end mainly in infragranular layers, but also in layer I. This is in accordance with previous studies (Leichnetz, 1989; Stanton et al., 1995). In addition, we find retrogradely tranduced cells, i.e., MT neurons projecting to area FEF, mainly in supragranular layers of MT. Several previous studies reported that MT neurons projecting to area FEF are mainly located in layer III of MT (Huerta et al., 1987; Leichnetz, 1989; Schall et al., 1995). 
In summary, our results show that optogenetics can be used to target the fronto-visual and fronto-parietal network. Stimulation experiments could be conducted in the injection areas as well as in distant areas that are targeted by axons of the injection area. 


\title{
Optogenetic inhibition of FEF input to area MT reduces attentional modulation of neuronal responses
}

\author{
Janina Hüer ${ }^{1}$ and Stefan Treue ${ }^{1}$
}

Additional scientists that contributed to the project in alphabetical order: Alexander Gail ${ }^{1}$, Jens Gruber ${ }^{3}$, Hansjörg Scherberger ${ }^{2}$, Lara Timantra Schiller $^{3}$ and Jochen Staiger ${ }^{4}$

\section{Affiliations}

1 Cognitive Neuroscience Laboratory, Deutsches Primatenzentrum GmbH, Göttingen, Germany

2 Neurobiology Laboratory, Deutsches Primatenzentrum GmbH, Göttingen, Germany

3 Medical RNA Biology, Deutsches Primatenzentrum GmbH, Göttingen, Germany

4 Department of Neuroanatomy, Georg-August University, Göttingen, Germany

Author contributions: JH and ST designed the experiment, JH recorded the data, JH and ST analyzed the data, JH and ST wrote the manuscript, JH, LTS, JG and JS were responsible for the viral vector testing and handling, $\mathrm{AG}, \mathrm{JH}, \mathrm{HS}$ and ST conducted the injections of the viral vector.

\section{Acknowledgements}

We thank Michael Feyerabend for helping with the virus testing. We thank Karl Deisseroth for the viral construct.

\section{Introduction}

Visual attention has been shown to modulate neural activity in the visual system of the macaque. Modulations have been observed in many visual areas, e.g., V1 (Motter, 1993; McAdams and Maunsell, 1999), V2 (Motter, 1993; 
Luck et al., 1997), V4 (Moran and Desimone, 1985; Motter, 1993), MT (Treue and Maunsell, 1996, 1999), and MST (Treue and Maunsell, 1996, 1999). This common finding raises the question how the neural activity in these areas is modulated. It seems likely that feedback signals originating in higher brain areas, like the parietal and prefrontal cortex, cause a modulation. Several studies indicate that the frontal eye field $(\mathrm{FEF})$, located in the prefrontal cortex, provides a feedback signal to visual areas that results in the attentional gain modulation of firing rates. This has primarily been shown for area V4 (Moore and Fallah, 2001; Moore and Armstrong, 2003; Moore, 2004; Armstrong and Moore, 2007; Gregoriou et al., 2009; Noudoost and Moore, 2011a; Gregoriou et al., 2012).

The most direct evidence stems from a study of Gregoriou et al. (2014) and shows a causal relationship of activity in the prefrontal cortex and attentional modulation in area V4. The authors lesioned the prefrontal cortex, including the FEF, of one hemisphere and evaluated the effect on attentional modulation of V4 neurons. Attentional modulation was reduced on average by $40 \%$ in V4 of the lesioned hemisphere compared to V4 of the non-lesioned hemisphere. In addition, latencies of attentional effects were longer and attentional enhancement of LFP gamma frequency power was lower in the lesioned hemisphere. This study showed that there is a causal relationship between activity in the prefrontal cortex and attentional modulation in area V4, but the method that was used was not able to differentiate whether these effects emerge via direct connections between the areas or via indirect pathways including additional brain areas. It is known from anatomical studies that the FEF sends direct neuronal projections to visual areas V4 and MT (Stanton et al., 1995; Anderson et al., 2011; Ninomiya et al., 2012). However, it is unknown whether this direct connection plays a role during attention and the modulation of firing rates in visual areas.

As mentioned above, most studies investigated the role of the FEF on the attentional modulation in area V4. Despite the existence of reliable evidence that attention modulates activity also in visual area MT (Treue and Maunsell, 1996; Treue and Martinez Trujillo, 1999; Treue and Maunsell, 1999), no study so far addressed the question of a causal role of the FEF in this area.

We wanted to shed light into the circuitry of attention. Therefore, we used 
pathway-specific optogenetics to manipulate the direct neuronal projection from the FEF to area MT. We injected a viral vector (AAV5- $\alpha$ CamKIIeNpHR3.0-mCherry) into the FEF of two rhesus macaques, bringing an inhibitory opsin into excitatory neurons. We used an opsin that has been shown to be incorporated into the membrane of axons and has successfully been used to inhibit axonal projections before (Gradinaru et al., 2010; Tye et al., 2011; Kim et al., 2013). Assuming that the opsin is incorporated into the neuronal membrane, including long-range axonal projections, we optically stimulated FEF axons in area MT of one of the monkeys while the animal was conducting a spatial attention task. The approach of axon stimulation has been successfully used with excitatory opsins in two other studies in the non-human primate (Inoue et al., 2015; Galvan et al., 2016). In both studies, projections from cortical to subcortical regions were stimulated. Inhibition of axons has been successfully used in rodents (Tye et al., 2011; Kim et al., 2013), but not yet in non-human primates. Our approach, therefore, includes two methodological components new to experiments in non-human primates: optogenetic targeting of cortico-cortical projections and inhibition of projections.

The experiment allowed answering two yet open questions. The first is whether the FEF plays a role in attentional modulation of area MT neurons. The second is whether the direct projection from the FEF to visual areas alone has a significant contribution during deployment of attention.

\section{Results}

Several months after virus injections, we started to record single-cell activity in area MT in combination with optical stimulation (figure 2.8); see tables S3$\mathrm{S} 5$ for further details about the recordings) in one animal (monkey $\mathrm{H}$ ). The monkey was performing a spatial attention task (figure 2.9), in which he was cued to attend to one of two moving random dot patterns (RDP): one was placed in the receptive field of the recorded neuron and the other isoeccentric at the other side of the fixation point in the other visual hemifield. This generated two attention conditions: the monkey was either attending to the stimulus inside the receptive field (AttIN) or to the stimulus outside of the receptive field (AttOUT). The animal was rewarded for responding to a direction change in the cued stimulus with the release of a proximity sensor and had 
A

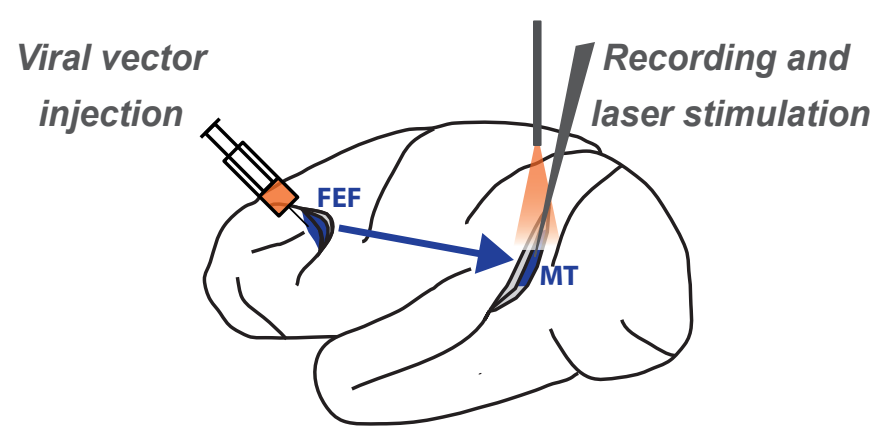

B
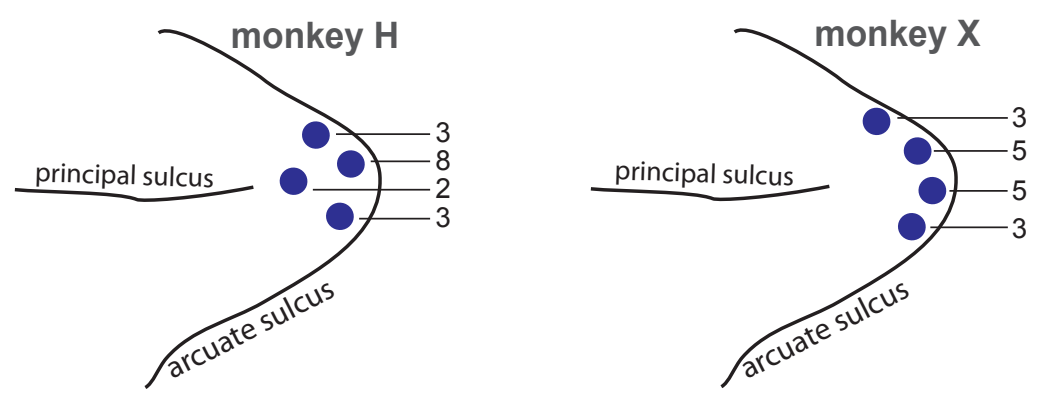

Figure 2.8: A. Experimental design. We injected a viral vector into the FEF of two monkeys, bringing an inhibitory opsin into the axons of FEF neurons. Electrophysiological recordings and laser stimulation of the FEF projections to area MT were conducted in area MT. B. Injection locations in monkey $\mathrm{H}$ and monkey X. Blue circles depict the injection locations. The values pointing to the locations specify the depths at which we injected in mm and number and the total injection volume in $\mu l$ (the value is the same for all three units). 
to ignore direction changes in the uncued stimulus. We optically stimulated during a period of stimulus presentation in which we expected attentional modulations of firing rates. Trials with and without laser stimulation were randomly interleaved, generating two stimulation conditions (noLaser, laser).

\section{Effects of laser stimulation on behavioral performance}

We compared performance between trials without and with laser stimulation (noLaser, laser) separately for the two attention conditions (AttIN, AttOUT). The hit rate in the AttIN condition was not changed by optical stimulation (mean across sessions $\mathrm{n}=42$, noLaser: 84\%, laser: 85\%, Wilcoxon signed-rank test $\mathrm{p}=0.27$ ). However, reaction times showed a small difference with optical stimulation (mean across sessions $\mathrm{n}=42$, noLaser: 361ms, laser: 356ms, Wilcoxon signed-rank test, $p<0.05)$. In the AttOUT condition, stimulation had a small effect on the hit rate (mean across sessions $n=42$, noLaser: $86 \%$, laser: $88 \%$, Wilcoxon signed-rank test $p<0.01$ ). In contrast, reaction times were not affected by stimulation (mean across sessions $\mathrm{n}=42$, noLaser: $382 \mathrm{~ms}$, laser: 380ms, Wilcoxon signed-rank test $\mathrm{p}=0.19)$.

\section{Attentional effects on firing rate over time}

We recorded 42 neurons, 38 were direction-selective (see method section for details). For each neuron, we recorded several pseudo-randomly interleaved conditions. Despite the AttIN, AttOUT, noLaser and laser conditions, we presented either 2 or 8 different directions. In the following report of results, we only analyzed the preferred direction.

Since we wanted to examine the effect of inhibiting the projection from the FEF to area MT on attentional modulation, we first determined how the attentional modulation developed over time during the progressing of a trial. It has been shown in other studies that the attentional modulation can be dependent on task expectancies of the monkey (Ghose and Maunsell, 2002) and can increase over the time course of a trial (Treue and Martinez Trujillo, 1999).

Without laser stimulation, the average attentional modulation (expressed by 
A

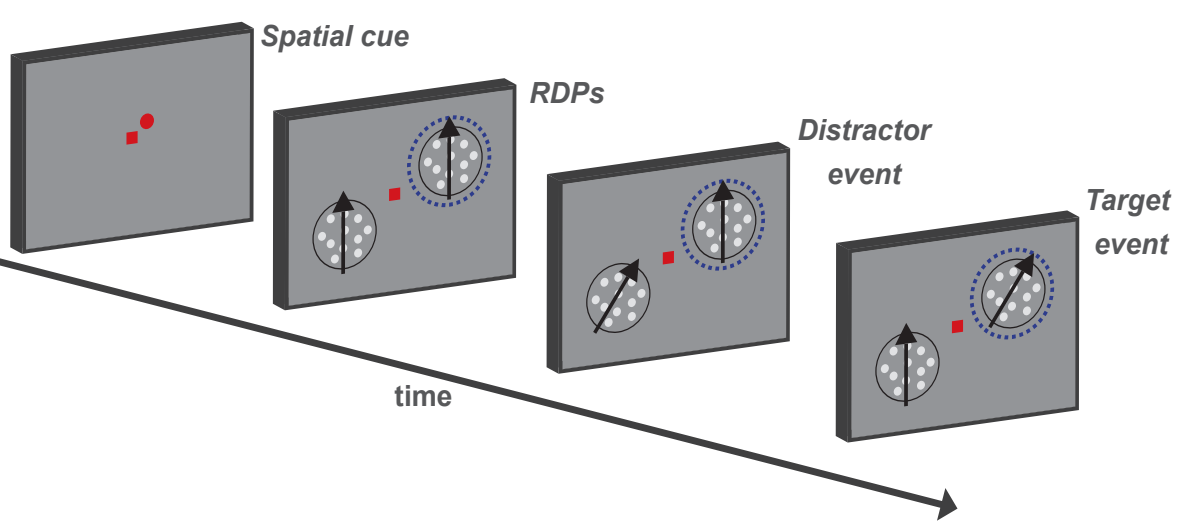

B

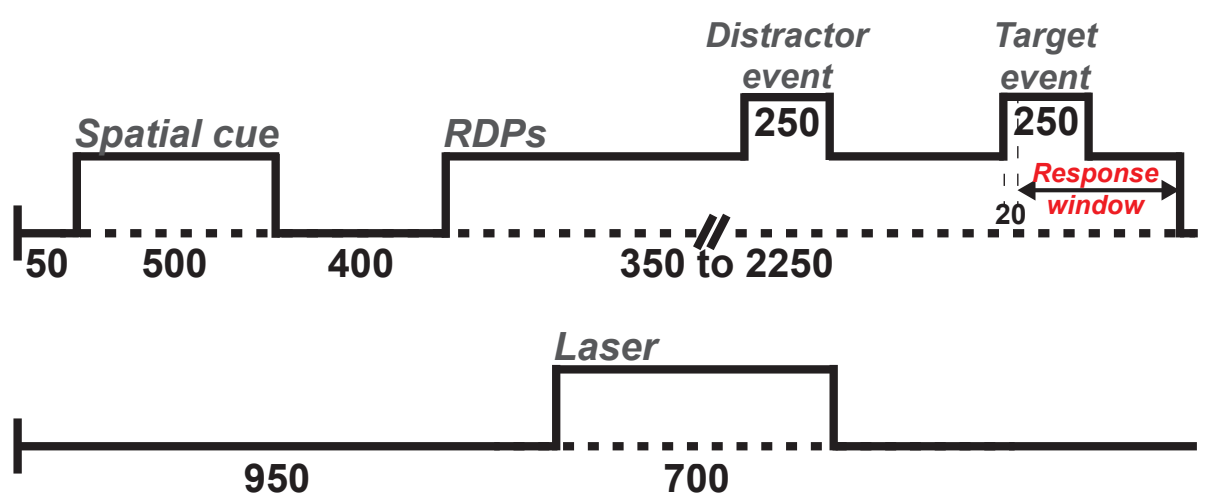

Figure 2.9: A. Behavioral task. A fixation point was shown in the middle of the screen. Monkeys had to fixate it and touch a proximity sensor. A cue appeared on the screen indicating which of the two RDPs was the target stimulus. Subsequently, the two RDPs were shown, one in the receptive field of the recorded neuron, the other in the opposite visual hemifield. The monkeys had to respond to a direction change in the cued RDP and ignore direction changes in the uncued RDP. B. Time course of trial events and laser stimulation. Direction changes in the target or distractor occurred between 350 and $2000 \mathrm{~ms}$ after stimulus onset. Therefore, trials had varying lengths. We stimulated with a continuous laser pulse of $700 \mathrm{~ms}$, starting $300 \mathrm{~ms}$ after stimulus onset. Durations of specific trial epochs are given in milliseconds. 

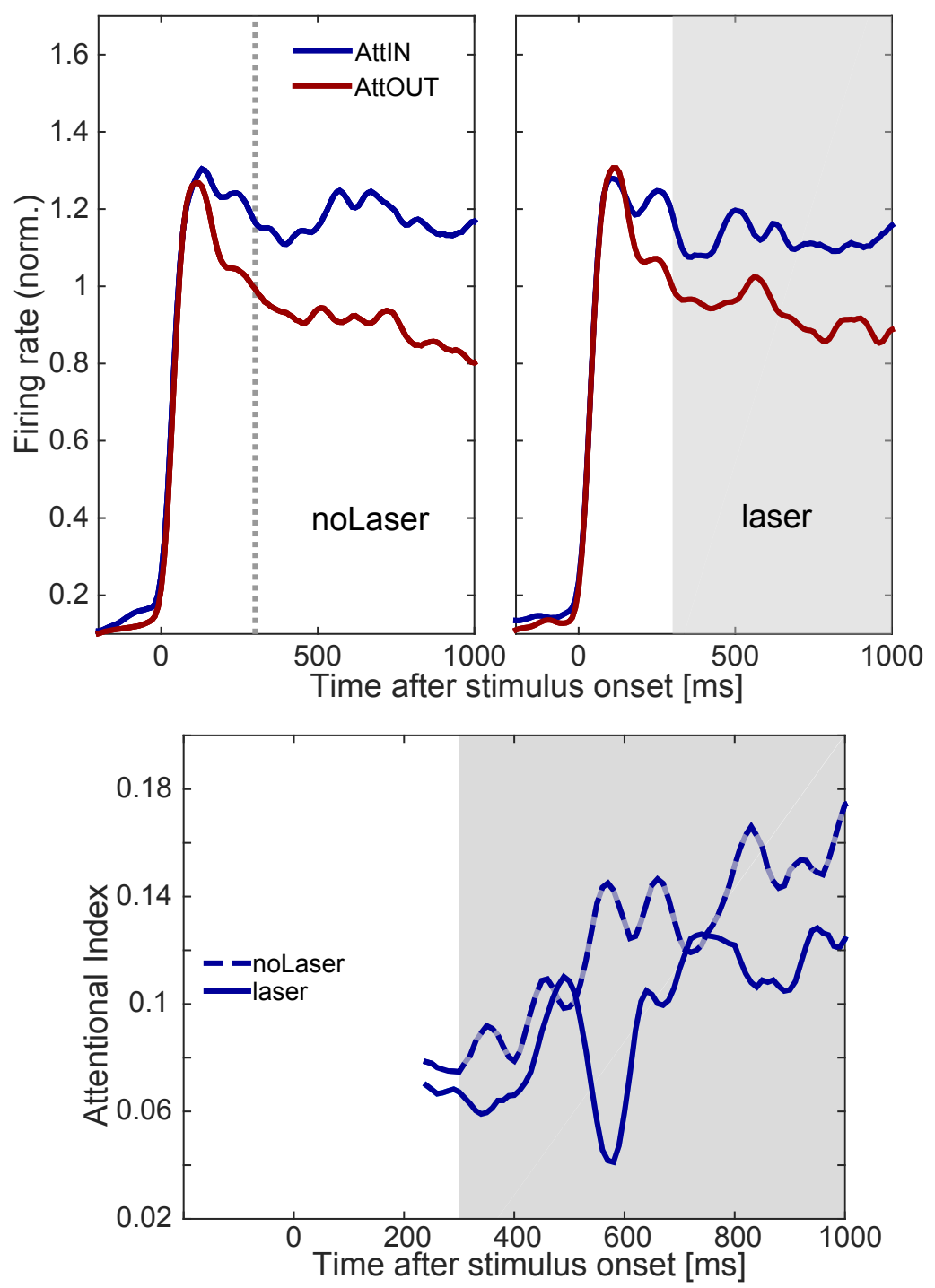

Figure 2.10: Time course of normalized firing rates without and with laser stimulation in the two attention conditions (AttIN, AttOUT) and time course of attentional indices without and with laser stimulation. 


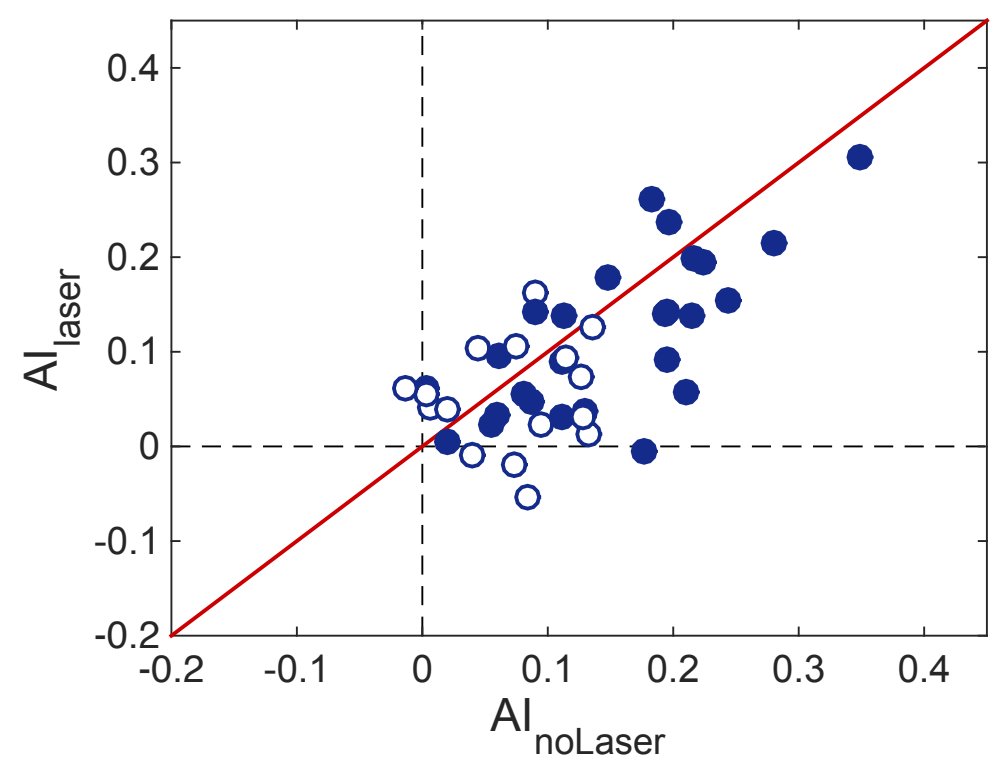

Figure 2.11: Attentional indices in the two stimulation conditions for each neuron in the time interval 300-1000ms after stimulus onset. Filled circles mark the neurons that showed a significant modulation with attention without laser stimulation.

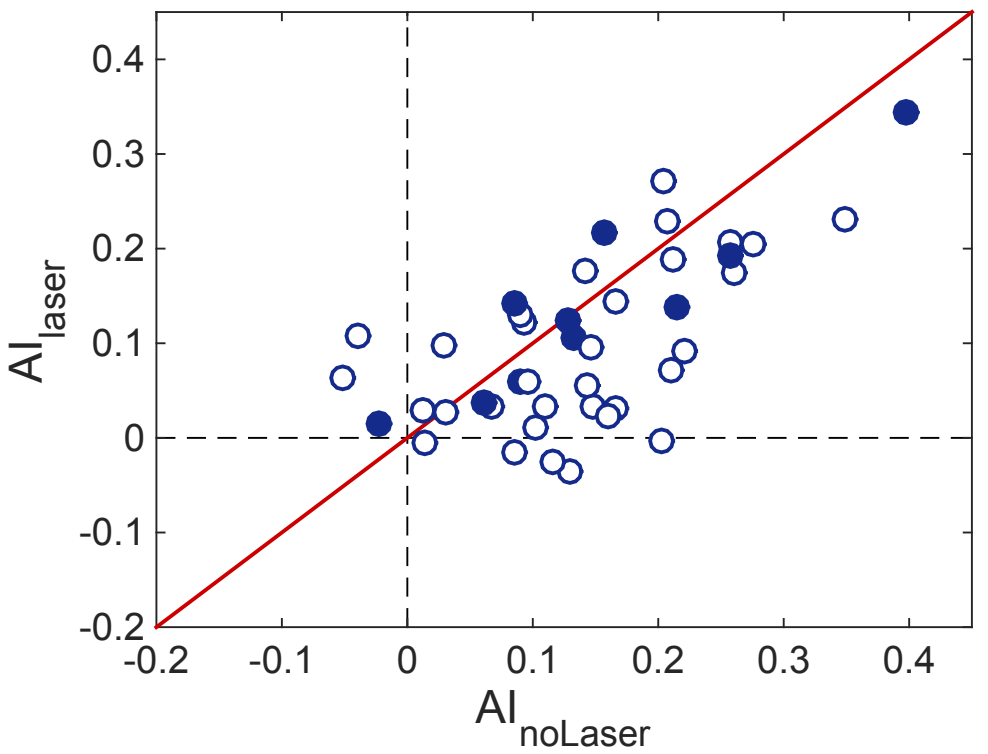

Figure 2.12: Attentional indices in the two stimulation conditions for each neuron in the time interval 540-1000ms after stimulus onset. Filled circles mark the neurons that showed a significant modulation with attention without laser stimulation. 


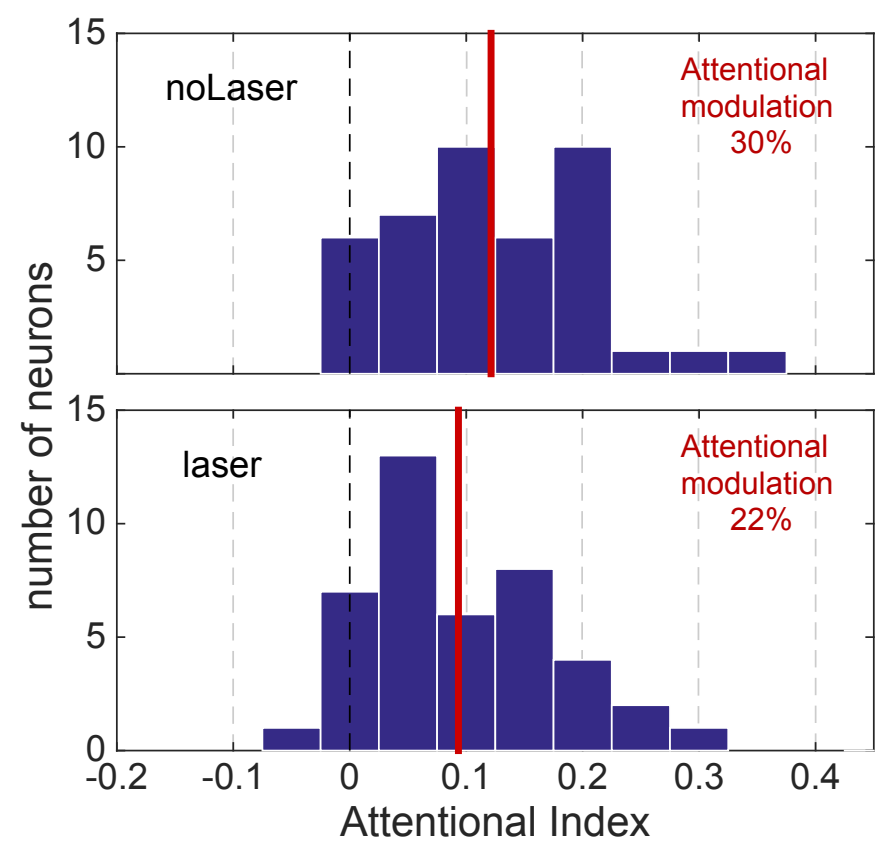

Figure 2.13: Distribution of attentional indices dependent on stimulation condition in the time interval 300-1000ms after stimulus onset. The red bar depicts the mean of the distribution. Attention increased responses by $30 \%$ in the noLaser condition and by $22 \%$ in the laser condition.

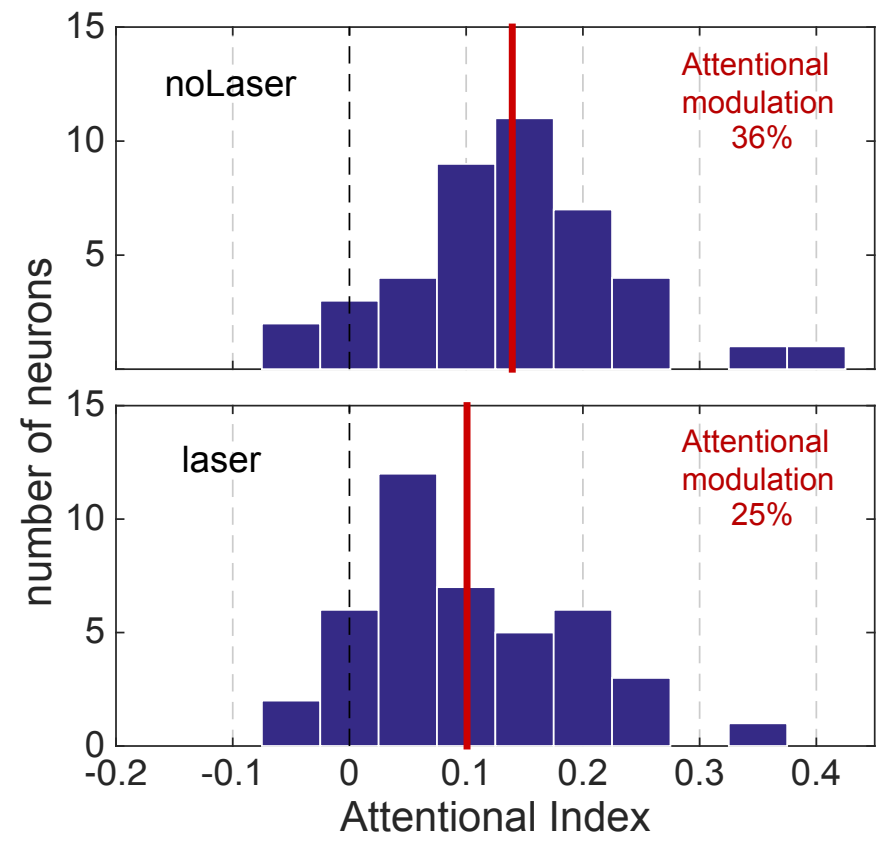

Figure 2.14: Distribution of attentional indices dependent on stimulation condition in the time interval $540-1000 \mathrm{~ms}$ after stimulus onset. The red bar depicts the mean of the distribution. Attention increased responses by $36 \%$ in the noLaser condition and by $25 \%$ in the laser condition. 


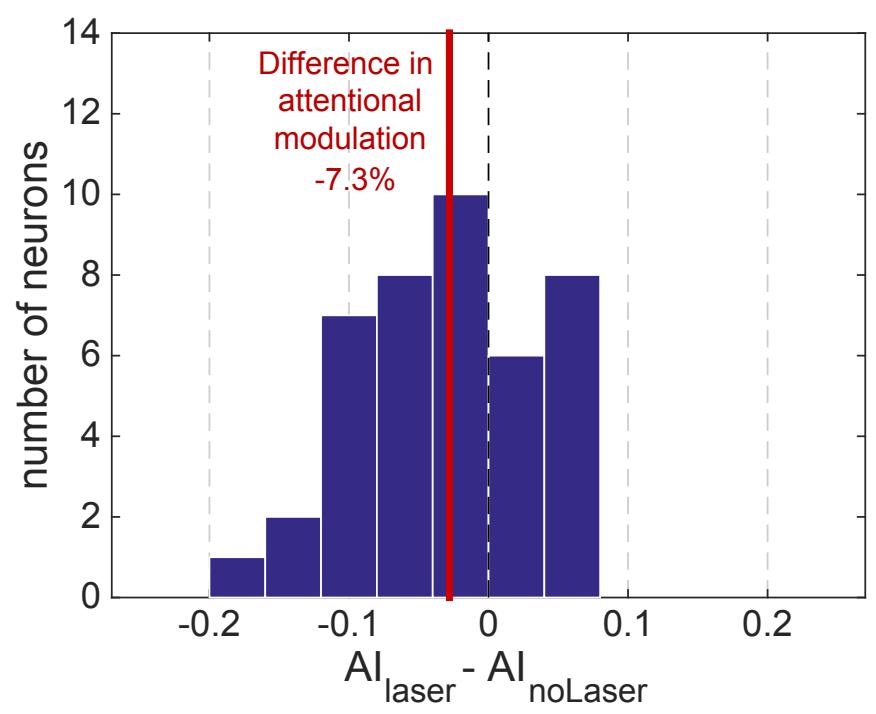

Figure 2.15: Difference in attentional modulation in the time interval 300-1000ms. The red bar shows the mean of the distribution. The absolute difference in attentional modulation was $7 \%$.

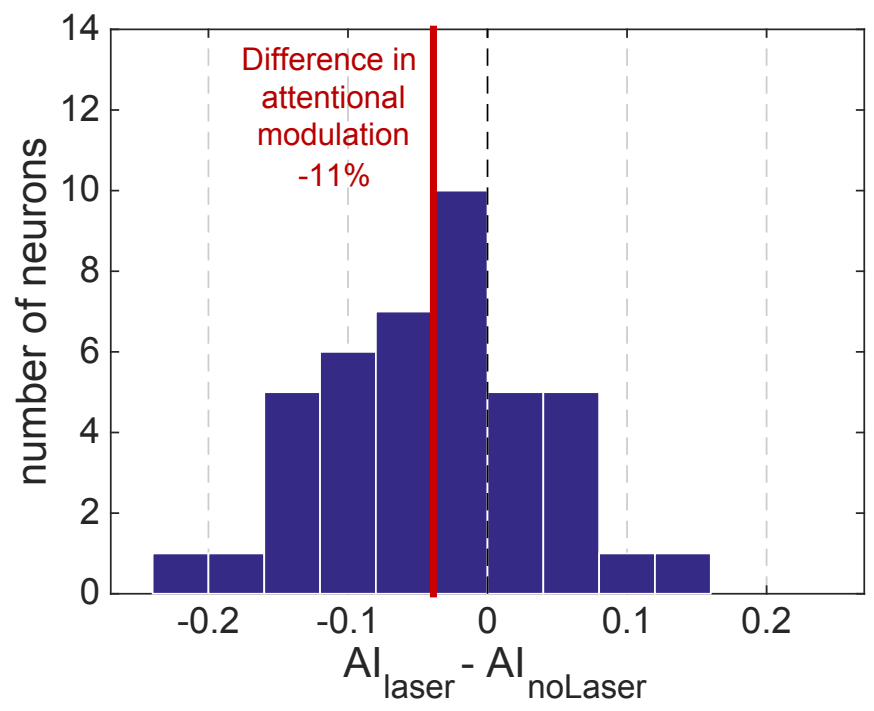

Figure 2.16: Difference in attentional modulation in the time interval 540-1000ms. The red bar shows the mean of the distribution. The absolute difference in attentional modulation was $11 \%$. 
attentional index, AI; see methods section for further details) increased over time (figure 2.10). After around $540 \mathrm{~ms}$ there was a pronounced increase in modulation. Therefore, we used two different time intervals for computing the AI: (1) 300-1000ms after stimulus onset and (2) 540-1000ms after stimulus onset.

\section{Effects of laser stimulation on attentional modulation}

We calculated an attentional index for each cell separately for the noLaser and laser conditions (see table S6 and S7). As mentioned above, we used two different time intervals. On the single cell level, 26 out of $42(62 \%)$ neurons showed a significant attentional modulation without stimulation in the period between 300 and 1000ms (figure 2.11, comparison of average response between AttIN and AttOUT condition, Wilcoxon rank-sum test, $p<0.05)$. With laser stimulation 22 neurons ( $52 \%$ of total cells) showed a significant attentional modulation. In the period of 540 to $1000 \mathrm{~ms}, 10$ out of 42 (24\%) neurons showed significant attentional modulation without stimulation (figure 2.12, Wilcoxon rank-sum test, $p<0.05$ ), and 11 neurons ( $26 \%$ of total cells) with laser stimulation.

To estimate the effect of laser stimulation on the population level, we compared the distributions of attentional indices (figures 2.13 and 2.14). Without stimulation, we found a mean increase of firing rates with attention by $30 \%$ (time interval 300-1000ms, AI: 0.12) and 36\% (time interval 540-1000ms, AI: 0.14). With optical stimulation attention increased firing rates by $22 \%$ (time interval 300-1000ms, AI: 0.09) and 25\% (time interval 540-1000ms, AI: 0.10 ). Inhibition of the projection from the FEF to area MT, hence, decreased attentional modulation by $27 \%$ (figure 2.15 , time interval $300-1000 \mathrm{~ms}$, Wilcoxon signed-rank test, $p<0.05$ ) and 31\% (figure 2.16, time interval 540-1000ms, Wilcoxon signed-rank test, $p<0.01)$.

\section{Effects of laser stimulation on firing rates}

To understand how stimulation changes firing rates in the two attention conditions (AttIN, AttOUT), we calculated a stimulation index (SI) for both conditions. Cells were differently affected by the stimulation (figures 2.17 and 


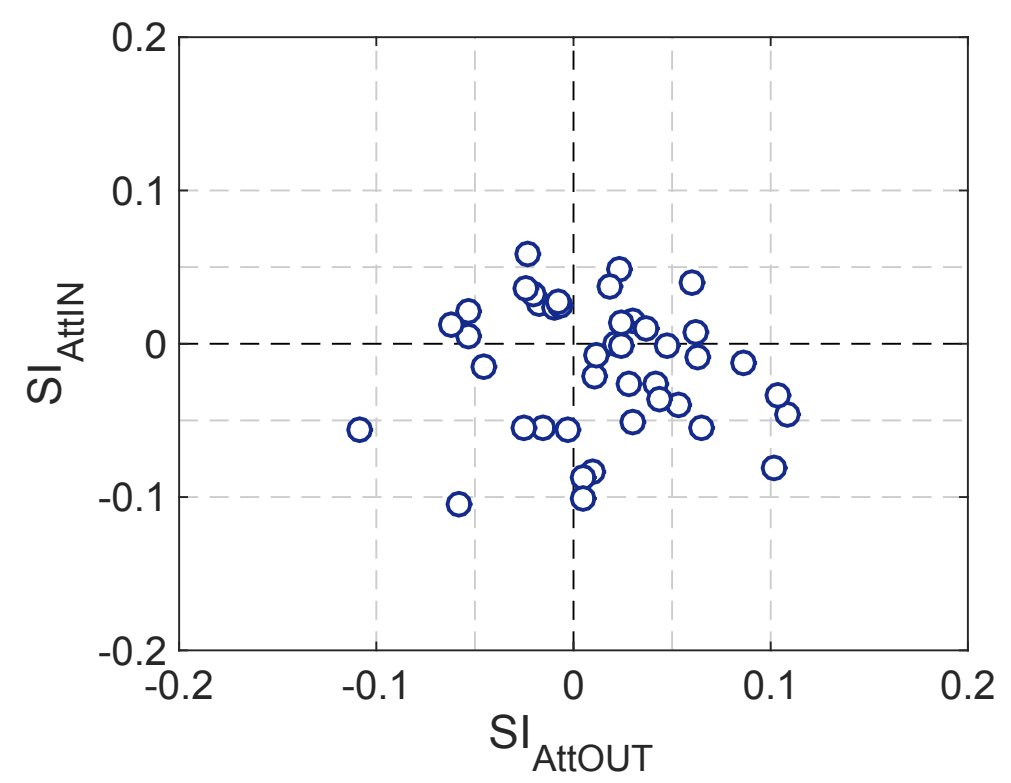

Figure 2.17: Stimulation indices in the two attention conditions for each neuron in the time interval $300-1000 \mathrm{~ms}$ after stimulus onset.

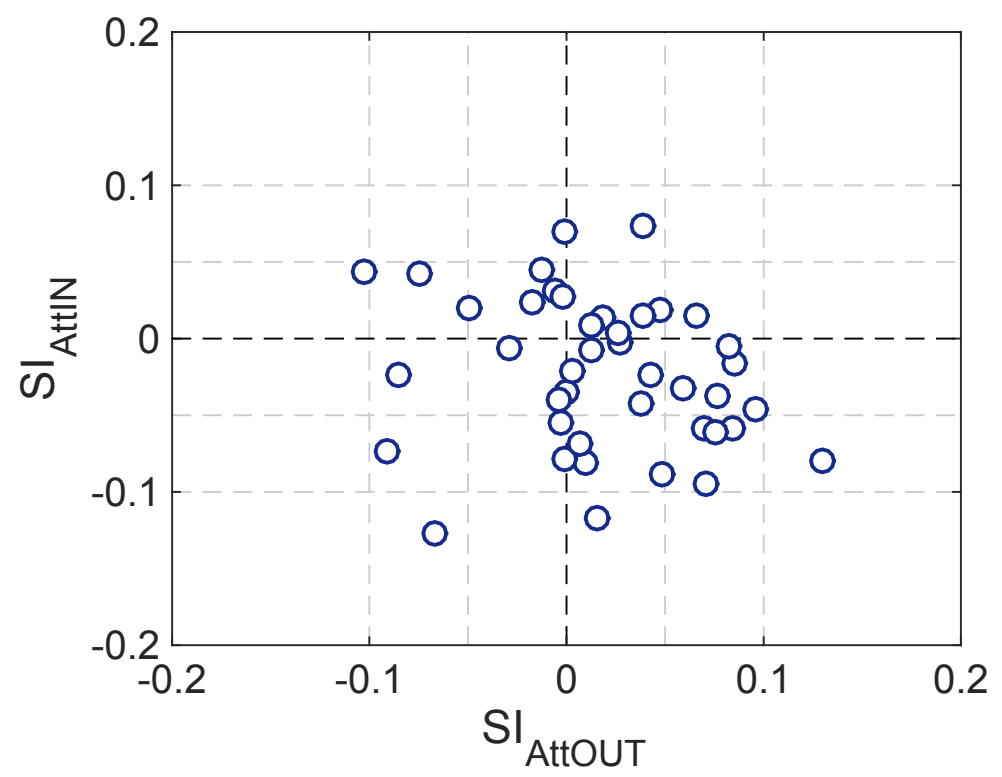

Figure 2.18: Stimulation indices in the two attention conditions for each neuron in the time interval 540-1000ms after stimulus onset. 


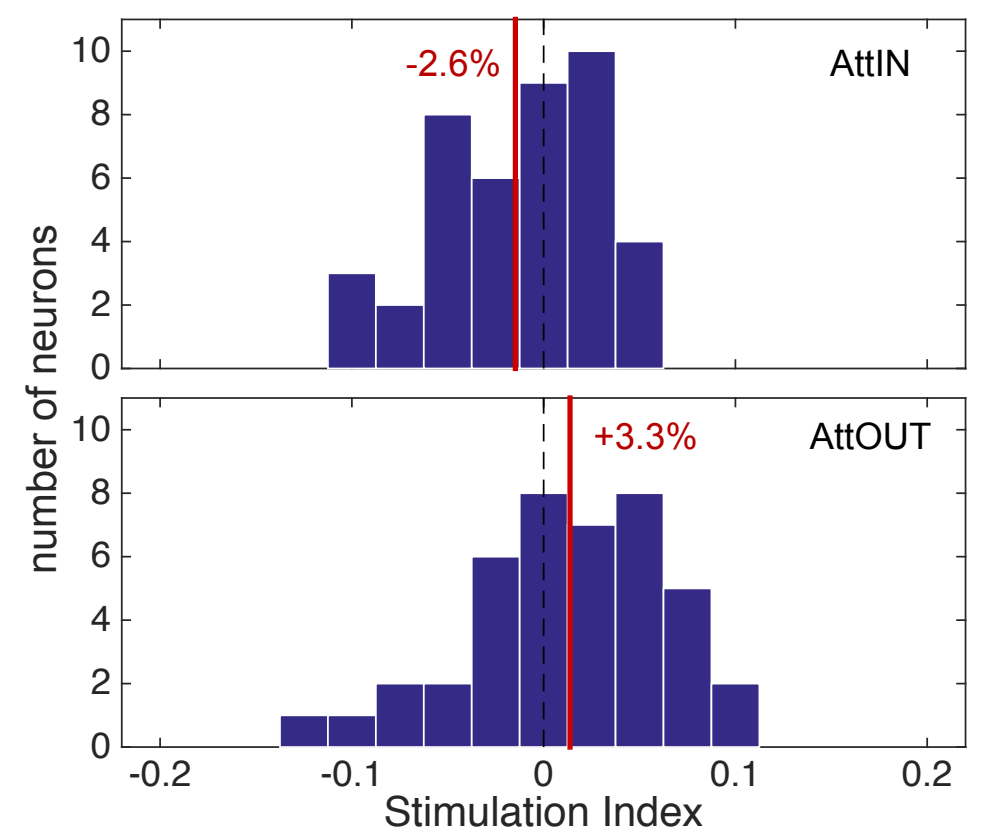

Figure 2.19: Distribution of stimulation indices in the two attention conditions in the time interval $300-1000 \mathrm{~ms}$ after stimulus onset. The red bar depicts the mean of the distribution. Laser stimulation decreased firing rates by $3 \%$ in the AttIN condition and increased firing rates by $3 \%$ in the AttOUT condition.

2.18), that is, cells can be found in all quadrants of the scatterplot. On the single cell level, only 5 cells were significantly affected by stimulation in one of the two attention conditions (both time intervals, Wilcoxon rank sum test, $p<0.05)$. Albeit not significant for a majority of single cells, stimulation decreased firing rates in the AttIN condition by 3 to $4 \%$ and increased firing rates in the AttOUT condition by 3 to $4 \%$ on the population level (figures 2.19 and 2.20). These effects were significant for the time interval $540-1000 \mathrm{~ms}$, but not for the time interval 300-1000ms (Wilcoxon signed-rank test, $p<0.05$ ).

\section{Discussion}

We used pathway-specific optogenetics to inhibit the projection from area FEF to area MT while a monkey was performing a spatial attention task. We find that optical stimulation significantly reduces average attentional modulation of area MT neurons by 27 to $31 \%$. We show that stimulation of FEF projections decreases firing rates when the monkey is attending to the stimulus in the receptive field, and increases firing rates when the monkey is attending to the 


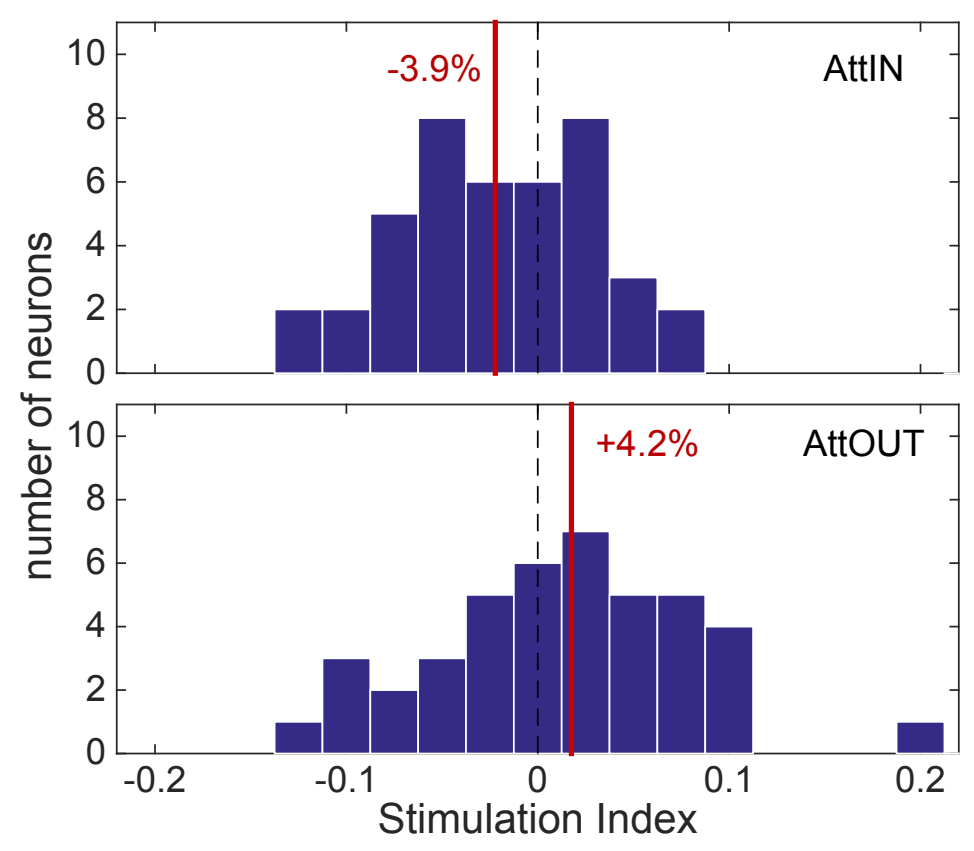

Figure 2.20: Distribution of stimulation indices in the two attention conditions in the time interval $540-1000 \mathrm{~ms}$ after stimulus onset. The red bar depicts the mean of the distribution. Laser stimulation decreased firing rates by $4 \%$ in the AttIN condition and increased firing rates by $4 \%$ in the AttOUT condition.

stimulus outside of the receptive field. This result suggests that there is pushpull mechanism of spatial attention mediated at least partially by FEF input. Relevant information is increased, while distracting information is decreased. This finding is in accordance with the results of previous studies. Moore et al. (2003) found that sub-threshold microstimulation of FEF neurons increased firing rates of V4 neurons when a target stimulus was shown in the receptive field of the neurons and the location of the receptive field matched the location of the response fields of the stimulated FEF neurons. In contrast, stimulation resulted in a decrease of firing rates when a distractor stimulus was shown in the receptive field. Further evidence for a push-pull mechanism stems from human fMRI studies (Rees, 1997; Pinsk, 2004), which found that the increase of activity related to target processing and the suppression of activity related to distracter processing was inversely related to the attentional load in these studies.

It is impossible to design an experiment in which a sensory stimulus is neither attended nor unattended, and the pure sensory response can be measured. 
Therefore, it is not possible to find out how neural firing rates would be without attentional effects in an awake animal. Our results indicate that the pure sensory response would lie between the attended and unattended response.

Without stimulation, we find that attentional modulation increased with the progressing of the trial. Firing rates were modulated shortly after stimulus onset, but the modulation increased sharply at around $540 \mathrm{~ms}$ after stimulus onset. Concurrently, attentional modulation with laser stimulation started to sharply decrease around the same time. We started stimulation 300ms after stimulus onset. It is unlikely that it takes $240 \mathrm{~ms}$ until stimulation effects become active. The latency of opsin activation has been shown to be less than 10ms (Mattis et al., 2012). Blocking already evoked action potentials within an axon, as we were aiming at in our experiment, might take more time, but it is implausible that the time difference would be more than $200 \mathrm{~ms}$. Rather the results suggest that there is a modulation of firing rates that is independent of FEF input, and that the effect of FEF on attentional modulation starts or is pronounced at around $540 \mathrm{~ms}$ in our task. It is likely that MT inherits part of its attentional modulation from an already modulated sensory input from lower areas, since neural activity can already by modulated by attention in V1 (Motter, 1993; McAdams and Maunsell, 1999). However, lesioning the prefrontal cortex (Gregoriou et al., 2014) resulted in a reduction of attentional modulation by $40 \%$, but not a complete abolishing of attentional modulation in visual area V4. The laser stimulation in our experiment can be assumed to reach only a limited spatial volume of tissue around the optical fiber tip. In addition, we probably did not reach all FEF neurons projecting to area MT with the viral vector injection. That means, we only partially inhibited the input from FEF to area MT. Therefore, it is not expected to see bigger effects on attentional modulation than with a complete lack of the FEF. However, the results of lesioning FEF indicate that the remaining attentional modulation in V4 could not be inherited from V1 assuming that all visual areas are modulated in a similar way. Therefore, it is likely that other cortical areas, like LIP, or cholinergic, dopaminergic or noradrenergic input plays a role in the modulation. Is has been shown, for example, that acetylcholine has an effect on attentional modulation in area V1 (Herrero et al., 2008). LIP shows a clear attentional modulation (e.g., Herrington and Assad, 2009; Ibos and 
Freedman, 2016), however its role as a potential source of top-down modulation in other visual areas still needs to be investigated.

Due to factors not related to the experiment, we started recording and stimulation not until nine months after viral vector injection and recorded the last cell 17 months after it. Even after this relatively long time after injection, we still found pronounced effects of laser stimulation on the attentional modulation of MT neurons. This indicates that FEF neurons and axons possess a robust expression of opsins and optogenetic experiments can be conducted over broad periods of time.

We find that laser stimulation has a small effect on behavioral performance or reaction time. However, the differences are so small that we do not consider them to represent a change in the animals perception at this point. Recording and stimulation in the second animal might clarify this finding.

The fact that stimulation had different effects on firing rates in different conditions indicates that systematic secondary effects, for example, heat produced by the laser, cannot explain our results. However, we cannot exclude the possibility that we partly stimulated MT neurons that project to the FEF and have taken up the viral vector retrogradely. We have shown in the project described in chapter 2.1 that after optogenetic injection in the FEF, retrogradely transduced cells can be found in several distant areas. Only a low number of neurons could be found in area MT. However, only a histological examination could clarify this concern.

We show for the first time, that FEF has a causal influence on attentional modulation in area MT. In addition, we show for the first time that a topdown long-range cortico-cortical projection directly modulates firing rates in a target area during a higher cognitive task in the non-human primate. Our results demonstrate that pathway-specific optogenetic inhibition can be used in non-human primates to expand our knowledge of the circuitry underlying complex behaviors. 


\section{Methods}

\section{Animals}

Two male monkeys (macaca mulatta) participated in this study. Monkey Harvey $(\mathrm{H})$ was 19 years old during the data collection; his weight was varying between 11 and $13 \mathrm{~kg}$ during the recording. Monkey Xaver (X) was 12 years old and weighing $11 \mathrm{~kg}$ at the point of virus injection. Monkey $\mathrm{H}$ was preferably using his left hand for the task, while monkey $\mathrm{X}$ was preferably using his right hand. The animals were group-housed in an animal facility of the German Primate Center. Both monkeys were alpha animals. They were continuously exposed to the natural day/night circle through windows and access to an outdoor cage. Diverse enrichment objects were present in the cages and exchanged by the animal caretakers on a regular basis. Each group was kept in sight with two additional monkey groups within one big room, so that communication was also possible between groups. Both monkeys had continuous free access to dry food. During the training and recording days they were gaining their fluid intake by juice reward during the experiment. The juice was chosen according to the animal's preference and usually switched from day to day between the most preferred juices (preferences: monkey $\mathrm{H}$ grape and pineapple juice, monkey X banana, grape and pear juice). Additional juices or mixtures of juices were tried occasionally to test the animal's preference and provide more variety. The animals could work as long as they wanted. They got an additional amount of fruits and vegetables each day, chosen according to the animal's taste preference, and a portion of protein-containing insects, cereals, nuts or yoghurt. Whenever no training or recording was conducted, monkeys had free access to water and got additional fruits and vegetables. The health of the monkeys was monitored daily by a veterinarian, animal caretakers, the responsible scientist and technical assistants.

All animal work and housing was conducted in accordance with all applicable German and European regulations. The scientists in this study are aware and are committed to the great respnsibility they have in ensuring the best possible science with the least possible harm to any animals used in scientific research. All animal procedures have been approved by the responsible regional government office (Niedersächsisches Landesamt fur Verbraucherschutz 
und Lebensmittelsicherheit [LAVES], Oldenburg, Germany) under the permit number 3392 42502-04-13/1100. All surgical and imaging procedures were done under appropriate anesthesia, with appropriate analgesics and in accordance with German laws governing animal use.

\section{Implants}

Monkey $\mathrm{H}$ was implanted with a titanium head post ten years before the experiment. He participated in other attention studies before. A peek recording chamber targeting area MT of the left hemisphere was implanted before the experiment. Monkey X was also implanted with a titanium head post.

\section{Viral vector injection}

We determined the location and the shape of the FEF with the help of an MRI scan and by anatomical landmarks (i.e., the arcuate and principal sulcus) after dura opening. A viral vector (AAV5- $\alpha$ CamKII-eNpHR3.0-mCherry, UNC Vector Core, titer: $4.7 \times 10^{12} \mathrm{vg} / \mathrm{ml}$ ) was injected into the left FEF of monkey $\mathrm{H}$ and the right FEF of monkey $\mathrm{X}$. We opened the dura in a surgery and

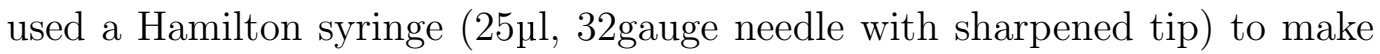
four penetrations (see figure 2.8). The distance between two penetrations was approximately $1.5-2 \mathrm{~mm}$. At each penetration, we injected at multiple depths. In monkey $\mathrm{H}$, we injected $1 \mu \mathrm{l}$ every $\mathrm{mm}$ with a speed of $200 \mathrm{nl} / \mathrm{sec}$. Starting with the deepest injection, we waited 5 minutes after each microliter to retract the tip of the syringe to the next depth. In monkey X, we injected $1 \mu l$ every $\mathrm{mm}$ with a speed of $300 \mathrm{nl} / \mathrm{sec}$, and waited 2-5 minutes before retracting the syringe to the next depth. The exact spatial configuration of the four penetrations was dependent on individual anatomy and the shape of blood vessels. Therefore, the configuration differed between both monkeys.

\section{Recordings and stimulation}

Stimulation experiments started several months (monkey H) after the injection (see table S3-S5 for details about the recordings). We recorded single-cell responses in area MT while optically stimulating in the vicinity of the electrode tip (figure 2.8). 
Area MT was localized by an anatomical MRI scan after chamber implantation and our recording sites were chosen based on the MRI scan. Prior to a recording and after isolating a neuron, we determined the position of the receptive field and the preferred direction of a neuron by a hand mapping using a moving RDP whose direction and position could be controlled by a computer mouse. If the preferred direction was not apparent with the hand mapping, we systematically presented different stimulus directions in the receptive field of a neuron, and determined the preferred direction based on an online analysis of the firing rate.

We used a multi-electrode manipulator (20-channel tetrode Mini Matrix System, TREC) with a concentric arrangement of five guide tubes for our recordings: a circle of four guide tubes surrounded a central guide tube. We placed

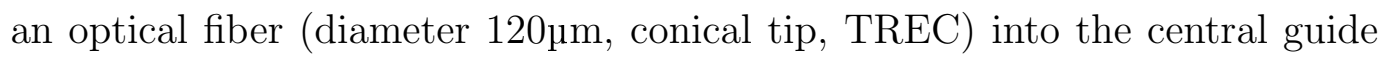
tube and four microelectrodes into the surrounding ones. The optical fiber was coupled to an orange (594nm) diode-pumped solid state (DPSS) laser (Cobolt) by an optical patch cable (105um, NA 0.22, Thorlabs). The laser power was controlled by an acousto-optical modulator (AOM). The AOM and the experiment were controlled by the software Mworks (version 0.6) running on an iMac (Apple Inc.). Neural data was recorded with an Omniplex system (Plexon Inc.). The eye position was monitored with an eyetracker (Eyelink 1000, SR Research). We analyzed whether there was a systematic difference between the gaze position of the monkey in the noLaser and laser condition. The mean difference was 0.02dva or less throughout the whole analysis period. Since receptive fields in area MT are much bigger, we assume that gaze position did not influence our results.

Before each recording session, we measured the light power at the end of the optical fiber tip. We used each optical fiber repeatedly in several sessions, but exchanged them whenever a major change in the light power at the tip occurred or when the glass body showed any sign of crack. We used a laser power that resulted in a measurable light power of $14 \mathrm{~mW}$ or $16 \mathrm{~mW}$ at the fiber tip (these values were based on the maximum laser power reported in Stauffer et al., 2016). Each optical fiber was usually used on several days without showing considerable change in the maximum output power. The optical fibers never broke as a result of the recordings. They had a sharpened 
tip, which resulted in an approximately circular light distribution around the tip. The power measured straight at the tip was usually higher than the laser power measured at the flanks of the tip. The tip length and shape differed slightly from fiber to fiber and therefore also the light distribution. We adapted the laser power for each recording session, so that the light power coming out straight at the tip was constant. The optical fiber tip was always placed above the tip of the recording electrode during the recordings. We used variable verticle distances of 0 to $800 \mu \mathrm{m}$ between the two tips (see table S5 for details).

\section{Behavioral task}

The animals were conducting a spatial attention task (figures 2.9). A red (size $0.2 \mathrm{x} 0.2 \mathrm{dva}$ ) fixation point was shown in the center of a computer monitor (BenQ XL2720T, resolution 1920x1080, refresh rate 120Hz). The animals had to foveate it and touch a proximity sensor (Carlo Gavazzi EC3016-NPAPL) in front of them to start a trial. A red circular cue (radius 0.3dva) appeared on the screen next to the fixation point after $50 \mathrm{~ms}$. The cue instructed the monkeys, which of the two subsequently presented moving random dot pattern (RDP) was the target stimulus. The cue was shown for $500 \mathrm{~ms}$ and followed by a blank period with only the fixation point present for 400ms. After it, two RDPs appeared, one in the receptive field of the recorded neuron, the other with the maximum distance at the same eccentricity in the opposite visual field. The size of the stimulus was adapted to cover the most responsive part of the receptive field. We used a motion direction pool of 8 directions $\left(0^{\circ}\right.$, $45^{\circ}, 90^{\circ}, 135^{\circ}$ etc.). For each neuron, we either recorded the full set of eight directions or we only recorded two directions (the one out of the direction pool that was closest to the preferred direction and the direction $180^{\circ}$ apart). Trials with and without laser stimulation, the movement direction and the target location were pseudo-randomly chosen. This resulted in $2 \times 2 \times 2$ conditions or $2 \times 8 \times 2$ conditions per recorded neuron. The monkey had to respond to a direction change of $25^{\circ}$ to $45^{\circ}$ in the target stimulus by releasing the proximity sensor, a direction change in the non-cued stimulus had to be ignored. The monkey was rewarded with a juice reward for completing the trial correctly. False alarms or misses were not rewarded. 1/6 of the trials were catch trials, 
in which no direction change occurred in the target stimulus. In this case, the monkey had to hold the proximity sensor until he received a reward.

We stimulated with a continuous pulse of $700 \mathrm{~ms}$, starting $300 \mathrm{~ms}$ after the onset of the two moving RDP. The direction change happened between $350 \mathrm{~ms}$ and $2000 \mathrm{~ms}$ after onset of the two RDPs.

\section{Data analysis}

We calculated performance and reaction time of the monkey. We differentiated between attention inside and outside of the receptive field (AttIN vs. AttOUT) and trials without and with laser stimulation (noLaser vs. laser). We did not differentiate between different directions for the calculation of the performance. Since we were interested in the effects of stimulation, we tested for differences in the noLaser vs. laser condition.

After the recordings, we first determined again offline whether the neurons were responding to the motion stimulus. We compared firing rates in the period of 300 to $200 \mathrm{~ms}$ before stimulus onset with the firing rates 200 to $300 \mathrm{~ms}$ after stimulus onset in the AttIN condition. All cells included in the analysis $(n=42)$ show significant differences (Wilcoxon signed-rank test, $p<0.05)$. We then determined whether cells were direction-selective. For that we compared firing rates in the period of 200 to $300 \mathrm{~ms}$ after stimulus onset between the presumably preferred and the opposite direction. 38 out of the 42 neurons were direction-selective (Wilcoxon rank sum test, $p<0.05$ ). For the 5 cells that were not direction-selective, we defined the direction that resulted in the highest firing rate during the period $200-300 \mathrm{~ms}$ after stimulus onset as the preferred direction.

We only analyzed successfully completed trials, only the trial period until the occurrence of any change in stimulus direction, and only the response to the preferred direction. Since trials varied in duration until the first stimulus change happened, for each cell and each condition (AttIN, AttOUT, noLaser, laser) we calculated a PSTH with a bin size of $10 \mathrm{~ms}$.

We calculated an attentional index $A I=\left(R_{A t t I N}-R_{A t t O U T}\right) /\left(R_{A t t I N}+R_{A t t O U T}\right)$ for each cell separately for the noLaser condition and laser condition by averaging the responses in the potential stimulation period based on the PSTH. We used two different time intervals (300-1000ms and 540-1000ms), and compared 
the distributions of attentional indices between the two stimulation conditions (noLaser and laser).

The use of two different time intervals resulted from an analysis of the time course of firing rates and attentional modulation with the progressing of the trial. We calculated the averaged normalized response dependent on trial time across all neurons and for the four conditions (AttIN, AttOUT x noLaser, laser). For each neuron, we binned the trials in $10 \mathrm{~ms}$ and divided the resulting firing rates of each bin by the average response of that neuron across all trials in the four conditions during 0 to $200 \mathrm{~ms}$ after stimulus onset. In addition, we calculated an AI for each bin by using the PSTH.

Analog to the attentional index, we calculated a stimulation index $S I=$ $\left(R_{\text {laser }}-R_{\text {noLaser }}\right) /\left(R_{\text {laser }}+R_{\text {noLaser }}\right)$ for each cell to test how stimulation affects firing rates in the two attention conditions (AttIN vs. AttOUT). 


\title{
An attentional blink with motion stimuli and in a task combining motion and letter stimuli
}

\author{
Janina Hüer ${ }^{1}$ and Stefan Treue ${ }^{1}$
}

Affiliations

1 Cognitive Neuroscience Laboratory, Deutsches Primatenzentrum GmbH, Göttingen, Germany

Author contributions: JH and ST designed the experiment, JH recorded the data, JH and ST analyzed the data, JH and ST wrote the manuscript.

\section{Acknowledgements}

We thank Nils Müller for conducting pilot experiments and Jonas Schweig for helping with the data collection and Sonia Baloni Ray for help in conducting all parts of the experiments.

The analysis of an initial part of this data was published in: Baloni, S. (2013), Spatial, feature and temporal attentional mechanisms in visual motion processing, http://hdl.handle.net/11858/00-1735-0000-0001-BA0F-B.

\section{Introduction}

Humans, like many other species with highly developed sensory systems have the ability to voluntarily attend to specific information in their environment. This selective attention confines processing to selected information, and is the basis for an efficient use of sensory processing resources. However, it also limits sensory perception.

When we try to detect a briefly presented white letter in a rapid serial presentation of otherwise black letters, the perception of letters following the white letter is impaired. This 'attentional blink'(Raymond et al., 1992) has two predominant characteristics: a top-down origin and a duration similar to an eye blink. In typical studies of the attentional blink (such as Raymond et al., 1992) subjects view sequences of visual stimuli in rapid serial visual presentation, with two target stimuli embedded within each sequence and with 
different time intervals between the two targets. The ability of humans to detect the second target is ideally determined as a function of inter-target interval in two conditions: a single-task in which subjects solely have to detect the second target and a dual-task in which they additionally have to identify the first target. While in the dual-task subjects often fail in detection, they show no impairment in the single-task despite the identical stimulus presentation. Processing of the first target triggers the impairment and interferes with the ability to detect or identify the second target for about 500ms. At later time points, subjects detect the second target equally well in single- and dual-task. The attentional blink, therefore, seems to represent the time attention stays on a target stimulus (the 'dwell time of attention', Duncan et al., 1994).

Most of the many studies of the attentional blink have used stationary visual stimuli (like letters or digits), which are processed by the ventral visual pathway (e.g., Livingstone and Hubel, 1987). We wondered if stimuli elicit the same temporary deficit in perception when they are processed in the dorsal visual pathway instead. The dorsal visual pathway is considered to be primarily involved in the processing of spatial information and planning and execution of actions (e.g., Goodale and Milner, 1992), including visual motion processing (e.g., Dubner and Zeki, 1971). Therefore, we chose moving random dot patterns (RDPs) as stimulus for our experiments. The sensory input to the dorsal visual pathway comes mainly from magnocellular cells of the lateral geniculate nucleus (e.g., Maunsell et al., 1990) - cells that are sensitive for luminance contrast and temporal frequency (e.g., Shapley et al., 1981; Merigan and Maunsell, 1990). In contrast, the ventral visual pathway receives its main input from the parvocellular cells of the lateral geniculate nucleus (e.g., Ferrera et al., 1994), which comprises cells that are sensitive for high spatial frequencies and color contrast (e.g., Merigan et al., 1991).

Despite this difference in sensory information input, similar top-down modulations have been observed in areas of both visual pathways. Visual attention modulates neural responses in areas of the dorsal pathway (e.g., Treue and Maunsell, 1996) as it does in areas of the ventral pathway (e.g., Moran and Desimone, 1985). Moreover, studies of object-based attention indicate that a unified attentional system operates globally upon the visual system. For 
example, two studies found that the response of neurons sensitive for features (color or motion) of an attended object that were irrelevant for the subjects ${ }^{6}$ task was enhanced even if the relevant feature was processed in the other visual pathway (Schoenfeld et al., 2003; Katzner et al., 2009).

As mentioned above, the attentional blink is not a sensory phenomenon, but rather shows a limitation in deployment of attentional resources (for a review see also Dux and Marois, 2010). Therefore, if processing of motion stimuli employs the same attentional resources, we expected that motion stimuli do cause an attentional blink and that an attentional blink influences processing in both visual pathways simultaneously.

We conducted four experiments to investigate a) if processing of motion stimuli causes an attentional blink and b) if an attentional blink elicited by a stimulus processed mainly in one of the two visual pathways affects perception of a stimulus processed by the other pathway in the same way.

\section{Methods}

\section{Subjects}

Twelve subjects with normal or corrected-to-normal vision participated in each experiment (Exp 1: 22 to 31 years, mean 26.6 years, 8 female; Exp 2: 21 to 31 years, mean 26.6 years, 6 female; Exp 3: 20 to 31 years, mean 26.9 years, 5 female; Exp 4: 19 to 31, mean age 25.6 years, 7 female, see tables S8-S15 for details about subjects). Except for one subject in experiments 3 and 4, subjects were naive to the aim of the experiment. Subjects provided informed written consent before the experiment and received a monetary compensation for their participation.

\section{Equipment}

All experiments were written and executed with the open source software MWorks on a MacPro (2x2.4 GHz Quad-Core Intel Xeon). The stimuli were shown on a 22" TFT monitor (Samsung SyncMaster 2233RZ) with a resolution of $36 \mathrm{pix} / \mathrm{deg}$ and a frame rate of $120 \mathrm{~Hz}$. The subjects were sitting in a quiet, dimly lit room; a chin and forehead rest stabilized their heads at a distance of $57 \mathrm{~cm}$ from the monitor. They received verbal instructions and gave their 
responses using a gamepad (Logitech Precision). In experiment 2, 3, and 4 eye positions were recorded with a video-based eye tracker (EyeLink 1000, SR Research). Subjects had to maintain their gaze in a window of 4 dva $\mathrm{x}$ $4 \mathrm{dva}$ on a fixation point placed in the middle of the screen for the duration of stimulus presentation; trials in which the gaze deviated from that window were repeated.

\section{Stimuli and procedure}

We adapted the design of Experiment 2 of Raymond et al. (1992) for the use with moving RDPs (figure 2.21). The general design was as follows. A gray $\left(9.15 \mathrm{~cd} / \mathrm{m}^{2}\right)$ box (height $12.5 \mathrm{dva}$, width $\left.16.3 \mathrm{dva}\right)$ was presented centered on a dark $\left(0.1 \mathrm{~cd} / \mathrm{m}^{2}\right)$ background. Subjects started each trial by pressing a start button on the gamepad; a white $\left(33 \mathrm{~cd} / \mathrm{m}^{2}\right)$ fixation point (height $0.2 \mathrm{dva} \mathrm{x}$ $0.2 \mathrm{dva}$ ) appeared for $183 \mathrm{~ms}$ in the middle of the gray box. Subsequently, a sequence of letters, random dot patterns (RDPs), or letters and RDPs was shown in RSVP in the middle of the gray box. Subjects had to give a response at the end of each trial.

The letters had a height of 1dva and were of font type Arial. The RDPs were shown within a stationary and circular virtual aperture with a radius of $4 \mathrm{dva}$ (experiment 1, 2, 4) or 5dva (experiment 3) and consisted of 250 (experiment 1, 2, 4) or 391 (experiment 3) dots (diameter 0.2dva) moving coherently at a speed of $25 \mathrm{dva} / \mathrm{s}$. The dots received a random position at the beginning of an $\mathrm{RDP}^{\mathrm{s}} \mathrm{s}$ presentation. Dots that left the RDP reentered at a random position at its opposite side. The letters and the dots of the RDP were black $\left(0.1 \mathrm{~cd} / \mathrm{m}^{2}\right)$ except for one stimulus in each sequence, which was white $\left(33 \mathrm{~cd} / \mathrm{m}^{2}\right)$. Each sequence of stimuli contained either one or two target stimuli: target 1 (T1) was the white colored stimulus, target 2 (T2) appeared in half of the trials and was shown at different target onset asynchronies (TOA) following T1.

An experimental session started with a training phase, in which subjects were trained separately to determine $\mathrm{T} 1$, or to indicate whether $\mathrm{T} 2$ occurred in the stream. They participated in the main experiment if they solved both tasks with an accuracy of at least $75 \%$. The main experiment consisted of two tasks, one of which was the detection of T2 (single-task). The other was a dualtask in which subjects had to determine T1 and to detect T2. The order of 
$\mathbf{A}$

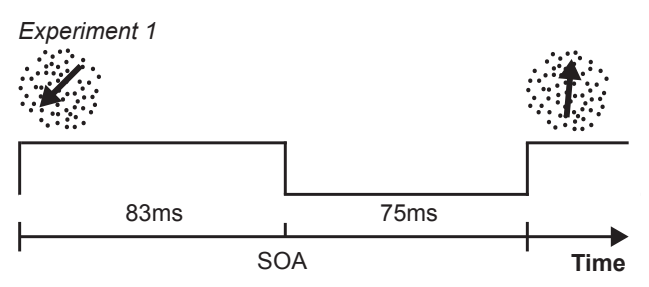

Experiment 2, 3, 4

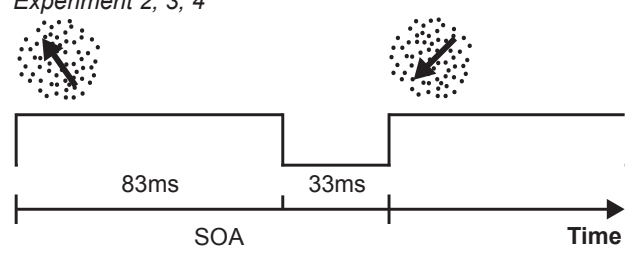

Experiment 3, 4

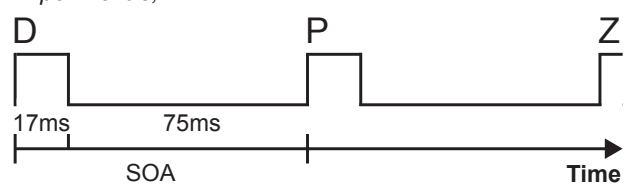

B

T1

Experiment 1, 2

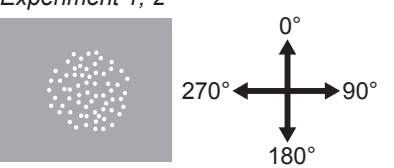

Experiment 3
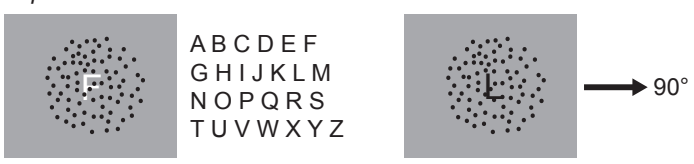

Experiment 4
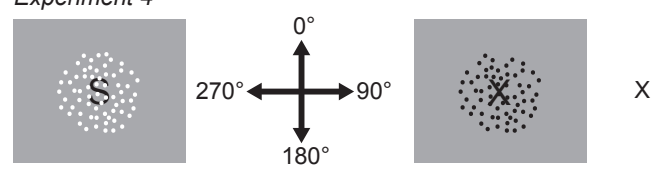

Figure 2.21: Procedure and stimuli. (A) RSVP time course. In experiment 1 and 2 , a sequence of RDP was presented. Each RDP was shown for $83 \mathrm{~ms}$ and followed by a blank period of $75 \mathrm{~ms}$ (experiment 1 ) or $33 \mathrm{~ms}$ (experiment 2), resulting in SOAs of $158 \mathrm{~ms}$ and $117 \mathrm{~ms}$. In experiment 3 and 4 , a combination of RDPs and letters was presented. The time course of the RDP stream was the same as in experiment 2 , the letters were shown for $17 \mathrm{~ms}$ and followed by a blank period of $75 \mathrm{~ms}$. (B) Target stimuli. In experiment 1 and 2, target 1 (T1) was a white RDP moving in one of four directions $\left(0^{\circ}, 90^{\circ}, 180^{\circ}, 270^{\circ}\right)$, target $2(\mathrm{~T} 2)$ was a rightward $\left(90^{\circ}\right)$ movement. In experiment 3, T1 was a white letter randomly chosen out of the 26 letters of the alphabet, T2 was a rightward $\left(90^{\circ}\right)$ movement. In experiment 4, T1 was a white RDP moving in one of four directions $\left(0^{\circ}, 90^{\circ}, 180^{\circ}, 270^{\circ}\right)$, T2 was the letter X.

executing both tasks was counterbalanced among subjects. Before conducting the dual-task, subjects got around 20 practice trials to get familiar with the task. During the whole session they could pause whenever they wanted, but were at least forced to make two breaks each of 1 minute during both singleand dual-task. 


\section{Trial course}

\section{Experiment 1 and 2}

Each trial consisted of a sequence of moving RDPs. The direction of a RDP's movement was randomly set to a value other then the range of $45^{\circ}-135^{\circ}$. Each RDP was shown for $83 \mathrm{~ms}$ (figure 2.21), which was the minimal presentation time necessary to enable acceptable performance. It was followed by a blank period of $75 \mathrm{~ms}$ (experiment 1) or 33ms (experiment 2), which resulted in two different stimulus onset asynchronies (SOA): 158ms (experiment 1) or $117 \mathrm{~ms}$ (experiment 2). Motion stimuli need to be presented longer than stationary stimuli to allow for sufficient integration over time (e.g., McKee and Welch, 1985). To estimate if this parameter has a substantial influence on our results, we tested these two different time courses. The number of RDPs varied between 12 and 17 (experiment 1) or 14 and 19 (experiment 2), so that a trial lasted between $2083 \mathrm{~ms}$ and $2875 \mathrm{~ms}$ (experiment 1) and between $1817 \mathrm{~ms}$ and 2400ms (experiment 2). T1 was a white colored RDP (figure 2.21). Its dots moved in one of the four cardinal directions $\left(0^{\circ}, 90^{\circ}, 180^{\circ}, 270^{\circ}\right)$, and its temporal position was randomly chosen between RDP 5 and 10 . Seven (experiment 1) or 9 (experiment 2) RDPs were always presented after T1. T2 was a RDP whose dots moved in the rightward $\left(90^{\circ}\right)$ direction (figure 2.21), and it was either the white RDP (experiment 1) or presented at one of the 5 (experiment 1) or 7 (experiment 2) temporal positions following it. In experiment 2, T2 was never the white RDP. Therefore, T1 could only move in the $0^{\circ}, 180^{\circ}$ or $270^{\circ}$ direction. Nonetheless, subjects were told that $\mathrm{T} 1$ could move in any of the four cardinal directions to make the experiment as similar as possible to experiment 1 . We accepted that difference in favor of reducing the number of trials, since subjects reported and showed signs of fatigue very rapidly in this task. In the single-task, subjects had to indicate whether T2 was presented. The dual-task was to determine the direction of T1 and to detect T2. Both tasks consisted of 180 (experiment 1) or 210 (experiment 2) trials. T2 was shown 15 times at each of the 6 (experiment 1) or 7 (experiment 2) possible temporal positions. An experimental session lasted about 70 (experiment 1) or 90 minutes (experiment 2) (including breaks). 


\section{Experiment 3}

Each trial consisted of a sequence of RDPs and a sequence of letters. The dots of the RDPs were spatially superimposed on the letters. The letters were chosen randomly out of the English alphabet, no letter occurred twice in a trial, and each letter was shown for $17 \mathrm{~ms}$ and followed by a blank period of $75 \mathrm{~ms}$. The movement directions of the RDPs were randomly set to a value other than the range of $45^{\circ}-135^{\circ}$, and the SOAs of the RDP sequence was the same as in experiment 2 (figure 2.21). T1 was a white letter chosen randomly out of the 26 letters of the alphabet, and T2 was a rightward movement. The time course of the RDP stream was dependent on the letter stream in the sense that the temporal position of $\mathrm{T} 2$ was adjusted to the onset of $\mathrm{T} 1$ or the 8 subsequent temporal letter positions. The whole RDP stream was shifted accordingly, so that there could be a temporal offset at the beginning of a trial. T1 was shown between position 8 and 14, and 11 letters always followed it. The number of letters shown determined the end of a trial, but an RDP presented at that time was always fully shown (including the following blank period). The number of RDPs consequently varied between 16 and 21 and a trial lasted between $1875 \mathrm{~ms}$ and $2425 \mathrm{~ms}$. In the single-task, subjects were instructed to detect $\mathrm{T} 2$, and in the dual-task to identify $\mathrm{T} 1$ and to detect T2. Both tasks consisted of 252 trials. T2 was shown 16 times at each of the 9 possible temporal positions. An experimental session lasted about 2 hours (including breaks).

\section{Experiment 4}

A sequence of RDPs and a sequence of letters with the same SOAs as in experiment 3 (figure 2.21) were shown. The direction of the RDP's movement was randomly set to a value between $0^{\circ}$ and $360^{\circ}$, the letters were chosen randomly out of the English alphabet. T1 was a white RDP that moved in one of the four cardinal directions, T2 was the letter X (figure 2.21). The temporal position of T2 was adjusted to the temporal position of the RDP at the position of $\mathrm{T} 1$ or the 6 subsequent temporal positions. The number of RDPs varied between 11 and 14. The position of $\mathrm{T} 1$ was 3 to 6 , and 8 RDPs always followed it. The number of RDPs shown determined the end of 
a trial, but a letter shown at that time was always fully presented (including the following blank period). The number of letters varied between 14 and 18 . The duration of a trial was between $1517 \mathrm{~ms}$ and $1867 \mathrm{~ms}$.

The single-task was the detection of $\mathrm{T} 2$, in the dual-task subjects were instructed to indicate the direction of $\mathrm{T} 1$ and to detect T2. Both tasks consisted of 224 trials. T2 was shown 16 times at each of the 7 possible temporal positions. One experimental session lasted about 90 minutes (including breaks).

\section{Data analysis}

We wanted to determine the influence of T1 identification on the ability to detect T2 as a function of the TOA. Therefore, we calculated the T2 detection rates in the single- and dual-tasks as the percentage of correctly detected $\mathrm{T} 2$ in trials in which T2 was present. In the dual-tasks, we only considered trials in which $\mathrm{T} 1$ was correctly determined and in the following always refer to this conditional T2 detection rate for the dual-tasks. To quantify the impact of $\mathrm{T} 1$ identification on detection of $\mathrm{T} 2$, we computed the difference between the $\mathrm{T} 2$ detection rates for each TOA in both tasks. We conducted a repeated-measure two-way ANOVA and multiple paired t-tests corrected by the Holm-Bonferroni method to test for a dependence of $\mathrm{T} 2$ detection rates on task and TOA.

Separately, to give a measure for comparing overall performance in the dualand single-tasks, we calculated the percentage of trials in which T1 was correctly determined ( $\mathrm{T} 1$ accuracy), the percentage of trials in which the presence or absence of $\mathrm{T} 2$ was correctly indicated (T2 accuracy), and the false alarm rate of $\mathrm{T} 2$ independent of $\mathrm{T} 1$ accuracy.

\section{Results}

The aim of our study was to investigate if a stimulus that is processed by the dorsal visual pathway can evoke an attentional blink and if an attentional blink influences different pathways in the visual system simultaneously. We used moving RDPs and examined in four experiments how processing of such a stimulus affects and is affected by the processing of moving and stationary stimuli. 


\section{Experiment 1}

In the first experiment, we adapted a previous experimental design (Raymond et al., 1992) to investigate whether motion processing can cause an attentional blink and impair subsequent motion perception. Subjects had to detect a rightward movement in the single-task, and to additionally discriminate the direction of $\mathrm{T} 1$ in the dual-task.

The overall T2 accuracy (includes trials with and without T2) in the singletask was $86 \%$, and mean false alarm rate $15 \%$ (ranging from $2 \%$ to $33 \%$ across subjects). In the dual-task, overall T2 accuracy accounted for $76 \%$, and mean false alarm rate for $20 \%$ (ranging from $2 \%$ to $43 \%$ between subjects). T1 accuracy in the dual-task was $85 \%$.

The single-task was designed to test for task-independent interference (such as masking) between the stimuli. Task-independent interference should be small or absent, since we were looking for task-dependent interference. In the single-task, we calculated T2 detection rates for trials in which T2 had been shown dependent on TOA. T2 detection rates in the dual-task were calculated for trials in which $\mathrm{T} 2$ had been shown and $\mathrm{T} 1$ had been determined correctly. Indeed we find that in the single-task, T2 detection rates were consistently high ( $80 \%$ or more) for all TOAs (figure $2.22 \mathrm{~A}$ ). In contrast, in the dualtask, T2 detection rates dropped transiently as low as 55\%. We conducted an ANOVA, which revealed a significant effect of task (single-task, dual-task; $\mathrm{F}(1,55)=14.23, p<0.01)$, a significant effect of TOA $(\mathrm{F}(5,55)=10.05, p<$ $0.01)$, and a significant interaction of task and TOA $(\mathrm{F}(5,55)=12.63, p<0.01)$. An attentional blink manifests in a transient difference of $\mathrm{T} 2$ detection rates in single- and dual-task. Figure 2.22B shows the difference between T2 detection rates dependent on TOA. T2 detection rates were significantly different for TOAs of $158 \mathrm{~ms}$ and $317 \mathrm{~ms}$, but not for longer TOAs (paired t-test, $p<0.05$ ).

\section{Experiment 2}

In the second experiment, we used the same design as in the first experiment, but changed the time course of the experiment: the blank period between subsequent stimuli was decreased to $33 \mathrm{~ms}$ (instead of $75 \mathrm{~ms}$ ). This change decreased overall performance. In the single-task, overall T2 accuracy was 83\%, 
A

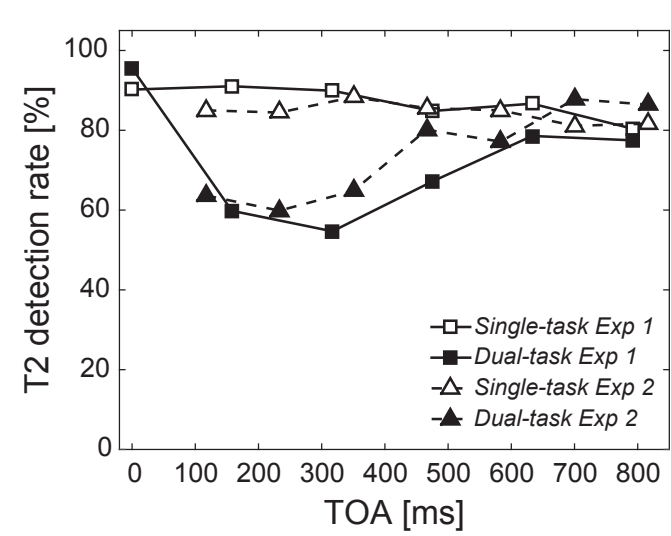

B

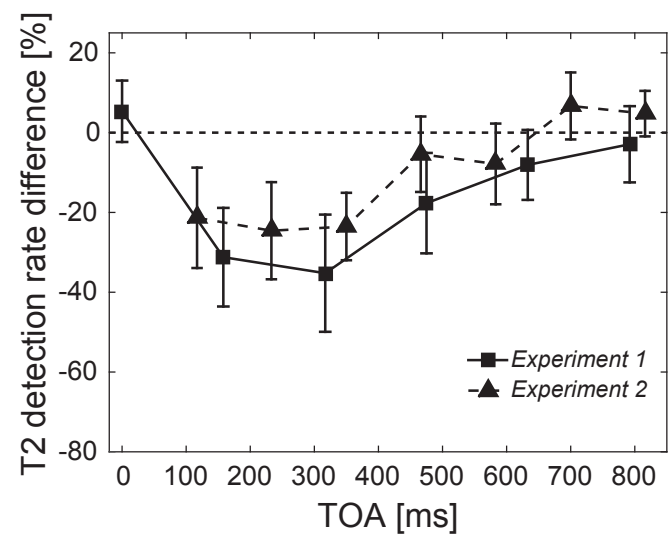

Figure 2.22: Results of experiment 1 and 2. (A) The T2 detection rates of both experiments are plotted as a function of TOA for the dual- and single-tasks. (B) The differences between the T2 detection rates in the dual- and single-tasks of both experiments are displayed as a function of TOA. The error bars show the $95 \%$ confidence interval of the difference.

and mean false alarm rate $19 \%$ (ranging from $1 \%$ to $31 \%$ ). In the dual-task, overall T2 accuracy accounted for $73 \%$, and mean false alarm rates for $29 \%$ (ranging from $13 \%$ to $43 \%$ ). The T1 accuracy in the dual-task averaged $76 \%$. Since these values do not provide information about the temporal dependency, we again calculated T2 detection rates for the single- and dual-task dependent on TOA. As in experiment 1, we did not find task-independent interferences. T2 detection rates were high ( $81 \%$ or more) for all TOAs. In the dual-task, T2 detection rates dropped to a minimum of $60 \%$ for a TOA of $233 \mathrm{~ms}$ (figure $2.22 \mathrm{~A}$ ). The ANOVA revealed a significant effect of task (single-task, dualtask; $\mathrm{F}(1,66)=13.98, p<0.01)$, a significant effect of $\mathrm{TOA}(\mathrm{F}(6,66)=3.48$, $p<0.01)$, and a significant interaction of task and $\operatorname{TOA}(\mathrm{F}(6,66)=8.50$, $p<0.01)$. The difference in T2 detection rates between single- and dualtask is shown in figure $2.22 \mathrm{~B}$. It was significant for TOAs up to $350 \mathrm{~ms}$, but not for longer TOAs (paired t-test, $p<0.05$ ).

\section{Experiment 3}

The third experiment was designed to test if an attentional blink evoked by stationary stimuli (letters) influences the processing of motion. Subjects had to conduct a single-task (detection of a movement [T2]), and a dual-task 
A

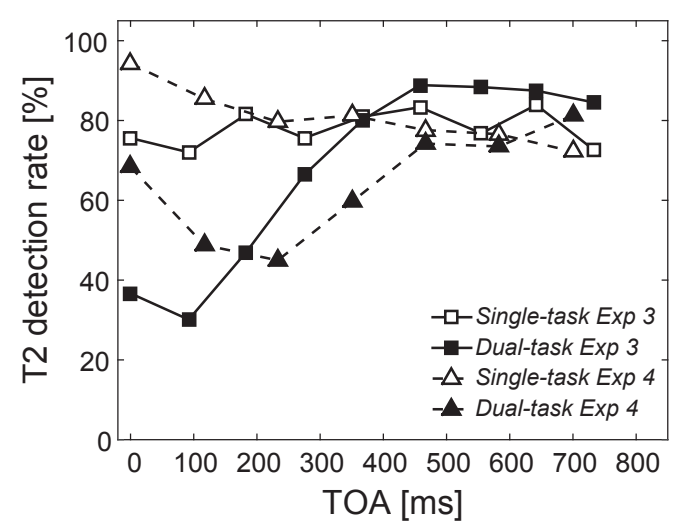

B

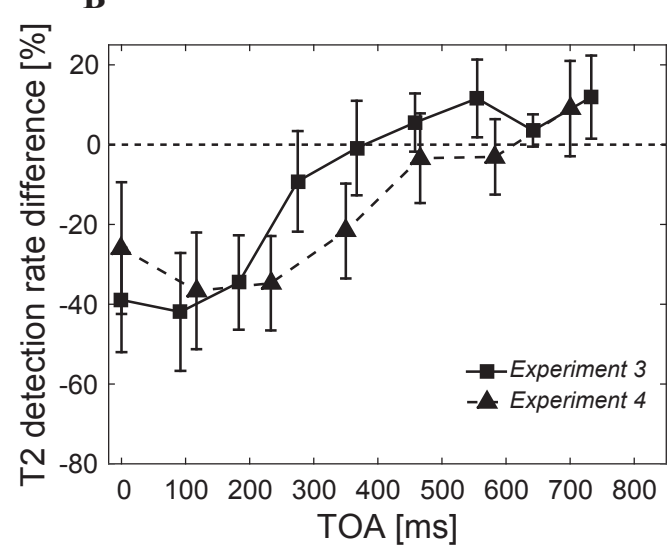

Figure 2.23: Results of experiment 3 and 4. (A) The T2 detection rates of both experiments are plotted as a function of TOA for the dual- and single-tasks. (B) The differences between the T2 detection rates in the dual- and single-tasks of both experiments are displayed as a function of TOA. The error bars show the $95 \%$ confidence interval of the difference.

(detection of $\mathrm{T} 2$ and identification of a letter [T1]). In the single-task, the overall $\mathrm{T} 2$ accuracy was $80 \%$, and the mean false alarm rate $18 \%$ (ranging from $4 \%$ to $28 \%$ ). In the dual-task, the overall T2 accuracy was $73 \%$, and the mean false alarm rate $22 \%$ (ranging from 2 to $33 \%$ ). T1 accuracy in the dual-task averaged $96 \%$. Figure 2.23A shows the T2 detection rates for single- and dual-task. Subjects detected T2 in $72 \%$ of trials or more for all TOAs in the single-task. In the dual-task, T2 detection rates were low for TOAs of $0 \mathrm{~ms}(37 \%)$ and $92 \mathrm{~ms}(30 \%)$, and reached the rates in the singletask for TOAs longer than $183 \mathrm{~ms}$. We conducted an ANOVA that revealed a significant effect of task $(\mathrm{F}(1,88)=18.83, p<0.01)$, a significant effect of TOA $(\mathrm{F}(8,88)=23.1, p<0.01)$, and a significant interaction of task and TOA $(\mathrm{F}(8,88)=16.60, p<0.01)$. The difference in $\mathrm{T} 2$ detection rates in single- and dual-task is shown in figure 2.23B. Detection rates differed significantly for TOAs up to $183 \mathrm{~ms}$ (paired t-test, $p<0.05$ ).

\section{Experiment 4}

The fourth experiment was conducted to test if an attentional blink evoked by processing of a motion stimulus influences processing of a subsequently shown stationary stimulus. Subjects again had to conduct a single-task (detection 
of a letter [T2]), and a dual-task (detection of $\mathrm{T} 2$ and determination of a movement direction [T1]). In the single-task, mean T2 accuracy was $88 \%$, and mean false alarm rate $5 \%$ (ranging from $0 \%$ to 15\%). In the dual-task, the mean accuracy of $\mathrm{T} 2$ detection was $76 \%$, and the mean false alarm rate $11 \%$ (ranging from 0 to $38 \%$ ). T1 identification in the dual-task was performed with a mean accuracy of $73 \%$. T2 detection rates were $73 \%$ or more for all TOAs in the single-task. They showed a U-shaped time course in the dual-task, starting with $68 \%$ for a TOA of $0 \mathrm{~ms}$, decreasing to a minimum of $45 \%$ for a TOA of 243ms, and reaching the rates of the single-task for TOAs longer than $350 \mathrm{~ms}$ (figure 2.23A). As expected, the ANOVA revealed a significant effect of task $(\mathrm{F}(1,66)=36.20, p<0.01)$, a significant effect of TOA $(\mathrm{F}(6,66)=6.46$, $p<0.01)$, and a significant interaction of task and TOA $(\mathrm{F}(6,66)=7.75, p<$ 0.01). T2 detection rates for TOAs up to $350 \mathrm{~ms}$ differed significantly between single- and dual-task (figure 2.23B, paired t-test, $p<0.05$ ).

\section{Discussion}

The aim of this study was to explore if processing of a motion stimulus can cause an attentional blink. Moving random dot patterns are processed by the dorsal visual pathway and thus constitute a type of stimulus that has marginally been used in attentional blink studies. Previous studies predominantly tested stationary visual stimuli, which are processed by the ventral visual pathway. We conducted two experiments to test how processing of a moving random dot pattern influences perception of subsequently shown moving random dot patterns. In two additional experiments we investigated how an attentional blink affects the two visual processing streams, i.e., the dorsal and the ventral stream. We combined stationary letter stimuli with moving random dot patterns and examined their interaction.

We show that processing of motion stimuli induces an attentional blink that affects both perception of motion and perception of stationary letters; equally, an attentional blink elicited by letter stimuli impairs perception of motion. A stimulus that is processed by the dorsal visual pathway, thus, can evoke an attentional blink. Moreover, an attentional blink seems to impact both visual processing streams, no matter which kind of stimulus caused the attentional blink, and, thus, seems to affect visual processing globally. 
Two previous studies showed already that processing of a stationary stimulus can affect processing of a motion stimulus in RSVP (Kawahara et al., 2001; Sahraie et al., 2001). However, in these studies stimuli were not shown at the same spatial location, and, therefore, the necessity to shift or increase the attentional focus in the spatial domain could also be a cause of the observed performance impairment. The stimuli in our experiment were spatially overlapping to reduce the impact that a shift in spatial attention might have on performance.

In experiment 1 and 2, we used two different SOAs (117ms and 158ms) to test how motion processing influences subsequent motion perception. The shorter SOA resulted in a slightly reduced attentional blink magnitude. This could simply be due to the variance in subjects ${ }^{6}$ performances or to the fact that shortening the blank period between sequential RDPs made the movement more continuous and therefore likelier to be perceived as a single object. Object continuity reduced or even abolished an attentional blink in studies of Raymond (2003) and Kellie and Shapiro (2004).

Several studies have suggested that the attentional blink is a consequence of the limited capacity of a late processing stage, and while the first target occupies this stage, the second target cannot be processed sufficiently (for a review see Martens and Wyble, 2010). However, other findings put the generality of this explanation in question. In about half (Visser et al., 1999) of all attentional blink studies subjects detected the second target more often when it was shown directly after the first target (i.e., at lag 1) than when it was shown at the following lags (this finding is known as 'lag-1 sparing", Potter et al., 1998), and additional studies extend this finding by demonstrating that subjects detect several targets as long as no intervening distractor is shown (Di Lollo et al., 2005; Nieuwenstein and Potter, 2006; Olivers et al., 2007; Potter et al., 2008; Raymond, 2003). Part of our results supports these results. In experiment 4 , the first target was a RDP and the second target the letter X. When they were shown simultaneously, subjects detected the second target in about $68 \%$ of trials, which was higher than chance performance $(50 \%)$ and higher than the performance for the three following TOAs. Even though the performance was lower than in the single-task, it means that in a majority of the trials subjects were able to process both targets simultaneously, and a 
capacity-limit as cause of the following detection deficit is implausible. Visser and colleagues suggested that the absence of lag-1 sparing in many studies arose by reason of the difference of the first and second target (Visser et al., 1999). They suggested thatt a filter and attentional settings are adjusted to the properties of the first target, and after its detection had to be reconfigured to process the second target. Since our stimuli necessitated very different processing but could still be processed simultaneously, our results contradict this explanation.

In contrast to experiment 4 , in experiment 3 , in which the first target was a letter and the second target a rightward movement, subjects' ability to detect the second target was lowest when both targets were shown simultaneously or directly following each other. This means that even though the stimulus types were similar in our experiments, the elicited perceptional deficits differed in their time course. While in experiment 1, 2 and 3 the duration of about 400ms was similar to that of most attentional blink studies, in experiment 4 the impairment lasted only about $200 \mathrm{~ms}$, and was similar to the results of a study of Sahraie et al. (2001). Instead of identifying a letter, subjects in their study had to detect a fixation point in a specific color. The second target task was a linear movement. As mentioned above, the two stimuli were not completely overlapping, instead the motion stimulus was surrounding the fixation point. However, the similarity in tasks and results and the difference to the time course in our experiment 3 indicate that magnitude and time course of the attentional blink to some extent display the needs to process the first, but also the second target.

Our results show that even though moving and stationary stimuli diverge in processing, they both can cause an attentional blink that impacts processing of both stimulus types similarly and simultaneously, supporting the hypothesis that a unified attentional system operates across the visual system. 


\title{
Pupil size suggests that the locus coeruleus is involved during attentional blink tasks without a direct perceptual consequence
}

\author{
Janina Hüer ${ }^{1}$ and Stefan Treue ${ }^{1}$
}

\begin{abstract}
Affiliations
1 Cognitive Neuroscience Laboratory, Deutsches Primatenzentrum GmbH, Göttingen, Germany
\end{abstract}

Author contributions: JH and ST designed the experiment, JH recorded the data, JH and ST analyzed the data, JH wrote the manuscript.

\section{Acknowledgements}

We thank Max Degener for conducting pilot experiments and helping with the data collection and analysis.

\section{Introduction}

Our ability to process information in parallel is limited. This limitation comes to the fore during a phenomenon known as the 'attentional blink ': visual processing of a chronologically second target (T2) is impaired when it is presented within 500ms after a first target (T1) (Raymond et al., 1992). Since Raymond et al. named the phenomenon in 1992, plenty of studies have dealt with it. Nevertheless, it is still unclear which neuronal mechanisms are underlying it. One of the many models explaining the attentional blink suggests that the locus coeruleus (LC) played a basic role in causing an attentional blink (Nieuwenhuis et al., 2005). The LC is a part of the brain stem, and it projects to almost all cortical areas, including areas considered to be involved in attentional processing (e.g., Morrison et al., 1982). It provides the major source of noradrenergic neurons to cortical areas (Berridge and Waterhouse, 2003), where it mainly binds to $\alpha 1$-adrenergic and $\beta$-adrenergic receptors (Arnsten, 2000). LC neurons respond to visual target stimuli with a phasic response (Aston-Jones 
et al., 1994; Usher, 1999; Murphy et al., 2014), which is thought to provide noradrenergic input to LCs recipient areas and modulate activity in sensory areas (Waterhouse et al., 1998). The phasic activity has been suggested to act as a temporal attentional filter supporting selective, task-relevant behavior (Aston-Jones and Cohen, 2005).

In the LC itself, noradrenaline exerts an inhibitory effect by binding to $\alpha 2$ adrenergic auto-receptors (Svensson et al., 1975; Egan et al., 1983; Washburn and Moises, 1989). Consequently, the phasic activity within the LC is often followed by a brief period of inactivity of LC neurons (Aghajanian et al., 1977; Aston-Jones et al., 1994; Usher, 1999). The duration of this refractory period (lasting several hundred milliseconds, Nieuwenhuis et al., 2005) is similar to the duration of an attentional blink. This similarity constitutes the basis for the suggestion of Nieuwenhuis et al. that activity in the LC is causing an attentional blink.

Nieuwenhuis and colleagues tested the role of noradrenaline in temporal and spatial attention (Nieuwenhuis et al., 2007). Subjects were administered the noradrenergic agonist clonidine, which acts as an agonist for the $\alpha 2$-adrenergic auto-receptors within the LC and thereby decreases activity within the LC (Aghajanian et al., 1977; Svensson et al., 1975). Subjects were conducting a temporal and a spatial attention task during a time window following drug administration in which clonidine exerts a physiological effect (Tiplady et al., 2005; Halliday et al., 1989; Coull et al., 2001). Contrary to the expectations, the authors did not find a difference in performance between treated subjects and a placebo group in an attentional blink task.

However, a study using the noradrenergic antagonist propanolol, which acts on the $\beta$-adrenergic receptors throughout the brain, showed a general reduction in the ability to either detect T1 or T2 (De Martino et al., 2008).

These results should be regarded with caution. As reviewed in Arnsten (2000), activation of different adrenergic receptors can have opposing effects in different brain areas. Therefore, global, unspecific administration of adrenergic drugs together with the temporal imprecision of this kind of drug administration can provide delusive results, and might mask the specific role of the LC. In recent years, a non-invasive physiological measure has more and more been used in the study of attention: tracking the diameter of the pupil. After early 
reports that the pupil size is influenced not only by sensory input, but also by higher cognitive processes like attention (Loewenstein, 1920; Hess and Polt, 1964), more recently, it has been suggested that pupil diameter can be used as an indirect measurement for LC activity in human subjects (Gilzenrat et al., 2010), and based on this assumption the role of the LC in visual processing has been investigated by measuring pupil size (Kihara et al., 2015).

A study in the non-human primate provided experimental evidence for the hypothesis that pupil size reflects LC activity (Joshi et al., 2016). The authors found that LC activity reliably preceded changes in pupil size. Additional brain areas showed the same relationship, but with longer latencies than the LC. Furthermore, a fMRI study in humans reported a correlation between pupil size and BOLD activity in the LC (Murphy et al., 2014).

As pupil size is influenced by attentional demands, Hoeks and Levelt (1993) developed a method with which changes of pupil size over time can be used to compute the potentially underlying attentional pulses. The method allows deconvolving the pupil size trace over time by using an Erlang gamma function as impulse response function, and delivers the underlying attentional pulses as an output. Parameters of the impulse response function have been determined experimentally by the authors.

Wierda et al. (2012) adapted this method for the application in tasks with high temporal frequency stimulus presentation. They analyzed pupil size during an attentional blink task and compared attentional pulses between blink and no-blink trials. Attentional pulses evoked by T1 presentation were higher in amplitude, and those evoked by T2 presentation lower in amplitude in blink tasks. The authors concluded that these results are consistent with a role of the LC during the attentional blink and pupil dilation.

In this study, we measured pupil size during a motion direction discrimination task that was based on the design of attentional blink experiments and the modified version used by Duncan et al. (1994). We used the deconvolution method (Wierda et al., 2012; Hoeks and Levelt, 1993) to indirectly test the hypothesis that LC activity is responsible for the occurrence of an attentional blink. Similar to our previous approach described in chapter 2.3, we compared a single-task (ST), in which only T2 had to be identified, and a dual-task (DT), in which $\mathrm{T} 1$ and $\mathrm{T} 2$ had to be identified, while presenting both target stimuli 
with varying target onset asynchrony (TOA). The two tasks were different only in the instruction the subjects received, but not in the visual sensory input, i.e., both target stimuli were shown in each trial. Therefore, our experimental design differed from previous studies that measured pupil size during attentional blink experiments (Wierda et al., 2012; Zylberberg et al., 2012), since in these studies the presented target stimuli were always relevant and attended, and no condition with an unattended, irrelevant target stimulus was conducted.

Our working hypothesis was that LC activity could be reconstructed by analyzing the pupil size with the deconvolution method. The resulting attentional pulses, under this assumption, would correspond to the LC input to the neuronal circuit controlling pupil size and resulting in a change of pupil size.

Our expectation was that attentional pulses evoked by the two target stimuli should differ between single- and dual-task concerning their amplitude. Pulses evoked by T1 presentation should be smaller in the single-task than in the dual-task, since in the former $\mathrm{T} 1$ is irrelevant and should not capture attention as much as in the dual-task. Furthermore, the attentional pulses evoked by $\mathrm{T} 2$ presentation should be smaller in the dual-task compared to the single-task within the period where an attentional blink typically occurs, because the LC is in its refractory period after T1 presentation. Pulses evoked by $\mathrm{T} 2$ presentation outside the attentional blink window should be similar in single- and dual-task.

\section{Methods}

\section{Subjects and experimental set-up}

All experiments were conducted in accordance with institutional guidelines for human experiments and the principles of the Declaration of Helsinki. Each subject gave informed written consent prior to the experiment and received a monetary compensation. In total, 26 naive subjects were trained in the task, out of which 14 subjects (female: 6, left-handed: 1, age: 19-45years; mean 25.5, see tables S16 and S17 for more information) reached a predefined criterion (performance of at least $75 \%$ in the single-task) within a few training sessions and participated in the main experiment. All subjects reported to 
have normal or corrected-to-normal vision. They were sitting in a quiet, dimly lit room. A chin and forehead rest stabilized their head, so that the subjects ${ }^{6}$ eye distance to the monitor was $57 \mathrm{~cm}$. Pupil size and gaze position were recorded with an EyeLink 1000 system (SR Research). The experiment was written with MWorks 0.6 and run on a MacPro $(2 \times 2.4 \mathrm{GHz}$ Quad-Core Intel Xeon). Stimuli were presented on a 22" LCD-monitor (Samsung SyncMaster 2233RZ) with a refresh rate of $120 \mathrm{~Hz}$ and a resolution of $1680 \mathrm{x} 1050$ pixel. Subjects gave their responses with a standard keyboard (Dell).

\section{Task design}

A red fixation point $\left(15.1 \mathrm{~cd} / \mathrm{m}^{2}\right)$ was presented in the center of a grey background $\left(21.6 \mathrm{~cd} / \mathrm{m}^{2}\right)$ (figure 2.24$)$. A black $\left(0.1 \mathrm{~cd} / \mathrm{m}^{2}\right)$ and a white $\left(79.2 \mathrm{~cd} / \mathrm{m}^{2}\right)$ moving random dot pattern (RDP) were shown at an eccentricity of 5 degrees of visual angle (dva). The black RDP was always located below the fixation point, the white RDP always above the fixation point. The RDPs consisted of 400 dots (radius 0.2dva) presented behind a circular virtual aperture of 3dva and moving with a speed of $6 \mathrm{dva} / \mathrm{s}$. Dots that left the circular aperture appeared at a random position at the opposite side of the RDP. Subjects started each trial by pressing a start button on the keyboard and fixating the central fixation point (fixation window 3dva). After 500ms the two RDPs appeared, one above and one below the fixation point. The dots moved in random directions. After 500-1000ms (pseudo-randomized) the black RDP (T1) started to move coherently into one of four possible target directions $\left(45^{\circ}, 135^{\circ}, 225^{\circ}\right.$, $315^{\circ}$ ) for $83 \mathrm{~ms}$. Subsequently, the coherent motion was replaced by a mask, in which each quarter of the dots moved into one of the four possible target directions. The mask was shown for at least $500 \mathrm{~ms}$, but always as long as the second stimulus was shown on the screen. The dots of the second, white RDP (T2) moved coherently into one of four target directions $\left(0^{\circ}, 90^{\circ}, 180^{\circ}\right.$, $270^{\circ}$ ) after a pseudo-randomized target onset asynchrony (TOA) of 0-700ms. The coherent motion lasted for $83 \mathrm{~ms}$ and was followed by a mask of $500 \mathrm{~ms}$. After both masks had been presented, both RDPs disappeared, but subjects had to keep fixation for $1500 \mathrm{~ms}$. At the end of the fixation period, a tone indicated that subjects were allowed to give a response. Trials were aborted and repeated, in case subjects pressed a button or lost fixation before trial 


\section{A}

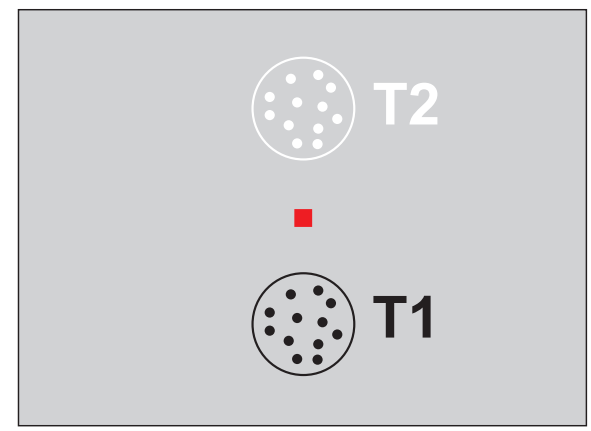

B
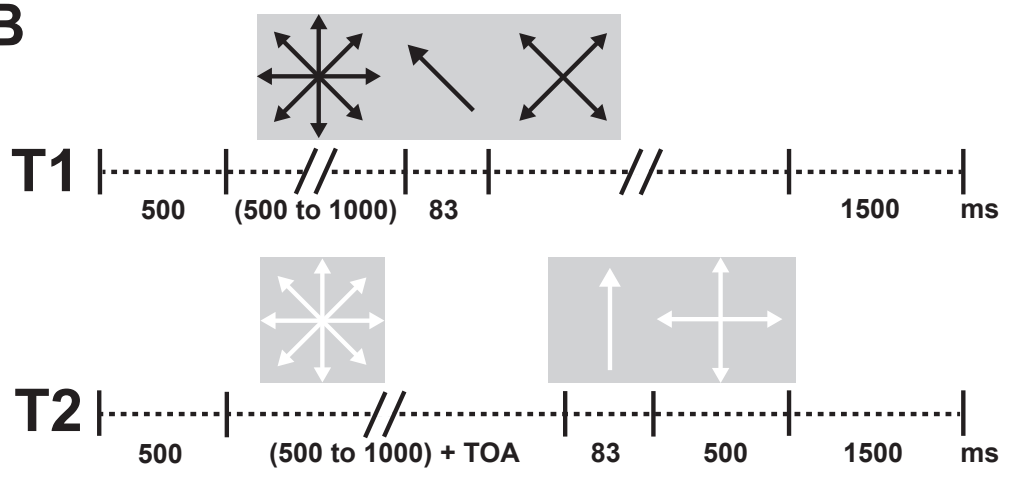

Figure 2.24: Task design. (A) Spatial arrangement of stimuli. T1 was always presented 5 dva below the fixation point, T2 always 5 dva above. (B) Time course of stimulus presentation. Numbers indicate duration in milliseconds. After 500ms of fixation, both stimuli simultaneously appeared on the screen. They differed in the time course of their intrinsic movement. Both stimulus streams started with the presentation of a randomly moving RDP. After 500 to $1000 \mathrm{~ms}$, T1 moved coherently for $83 \mathrm{~ms}$ into one of four possible directions and was followed by a mask for at least $500 \mathrm{~ms}$. T2 showed coherent movement after 0 to $700 \mathrm{~ms}$ after $\mathrm{T} 1$ onset and was followed by a mask of $500 \mathrm{~ms}$. Both stimuli disappeared after presentation of the T2 mask. Subjects continued fixation for $1500 \mathrm{~ms}$. 
end. Each subject conducted two conditions: a single-task and a dual-task. In the single-task, subjects had to indicate in which direction the second target stimulus (T2) was moving. In the dual-task, they had to identify the movement direction of both target stimuli (T1 and T2). Subjects conducted the two conditions block-wise and were always starting with the single-task. During a session each subject conducted two further tasks, which are not part of the following analysis.

\section{Data analysis}

All analyses were conducted in Matlab (MathWorks), except for the deconvolution modeling, which was done in $\mathrm{R}$ (version 3.2.1) using RStudio (version 1.0.143). We analyzed the overall performance of target identification in the different conditions. Furthermore, we determined the performance of T2 identification for the single- and dual-task in dependence of the TOA and compared it between the two tasks. T2 performance in the dual-task was only analyzed based on trials in which T1 was correctly identified.

The pupil size data was analyzed in the following way. For each trial we divided the pupil size at each point in time by the mean pupil size in the $500 \mathrm{~ms}$ period before $\mathrm{T} 1$ onset in that specific trial. Since we were interested in the influence of the target stimuli on the pupil size, we aligned the change of the pupil size to $\mathrm{T} 1$ onset time.

We compared pupil size between single-and dual-task in different ways. First, we averaged the pupil size over a period of 1000-2000ms after T1 onset and compared the mean between single-and dual-task based on trials with correct $\mathrm{T} 2$ identification (and additional correct $\mathrm{T} 1$ identification in the dual-task). We only used the correct trials, because we were interested in the pupil response elicited by the two target stimuli. In this way, we were as sure as possible that the subjects have seen the target stimuli and the measured pupil size was a result of target processing.

For the TOAs, in which performance was significantly different between singleand dual-task, we computed pupil size in the dual-task separately for correct ('no-blink trials') and incorrect ('blink trials') T2 responses based on trials with correct $\mathrm{T} 1$ identification. We again compared the averages over a period of 1000-2000ms after T1 onset between no-blink and blink trials. 
We used the deconvolution method as implemented by Wierda et al. (2012) and adjusted it to our needs. As described in Wierda et al. (2012), the method is based on the pupil deconvolution developed by Hoeks and Levelt (1993), but adapted to high frequency stimulus presentation. An attentional input $i=\omega_{1}, \omega_{2}, \ldots, \omega_{k}$ was deconvolved with an Erlang gamma function $h=s \cdot\left(t^{n}\right) \cdot e^{\left(-n \cdot t / t_{\max }\right)} \cdot \omega_{I}$ was the strength of the attentional pulses at the position $I$ in the vector $i, s\left(=1 / 10^{27}\right)$ a constant to scale the pupillary response. The parameters $t_{\max }(=930 \mathrm{~ms})$ and $n(=10.1)$ have been experimentally determined by Hoeks and Levelt (1993), and can be assumed to be stable over subjects and tasks. $T_{\max }$ was the temporal position of the maximum pupil response and $n$ the number of layers implemented in the model. We used the parameters of the Erlang gamma function as experimentally determined by Hoeks and Levelt. The predicted pupil dilation was calculated as $p d=I \cdot b+(i * h)$; where $b$ was a parameter accounting for the drift in the pupil data. The strength $\omega_{I}$ of the attentional pulses was fit by minimizing the mean square error by using the Nelder-Mead method.

With this analysis, we calculated the underlying attentional pulses for several conditions. For all conditions, we modeled 21 pulses starting at $-500 \mathrm{~ms}$ before T1 onset and ending 1400ms after T1 onset with an inter-pulse distance of $100 \mathrm{~ms}$. The Nelder-Mead method gives a slightly different result for each run. Therefore, we calculated the mean pulse strength out of 200 iterations.

We first analyzed trials independent of the TOA for trials with correct T2 identification (and additional correct T1 identification in the dual-task) and compared attentional pulses between single-and dual-task. Furthermore, we calculated attentional pulses dependent on TOA for trials with correct T2 identification (and additional correct T1 identification in the dual-task). In addition, we compared blink and no blink trials for a TOA of $100 \mathrm{~ms}$. We chose this TOA, because it resulted in the biggest difference in performance between single-and dual-task.

\section{Results}

We first analyzed the performance of the subjects. Overall, T1 was reported correctly in $82 \%( \pm 0.03 \%(\mathrm{SEM}))$ of trials in the dual-task. T2 was reported correctly in $75 \%( \pm 0.04 \%)$ of trials in the dual-task and $91 \%( \pm 0.02 \%)$ of 


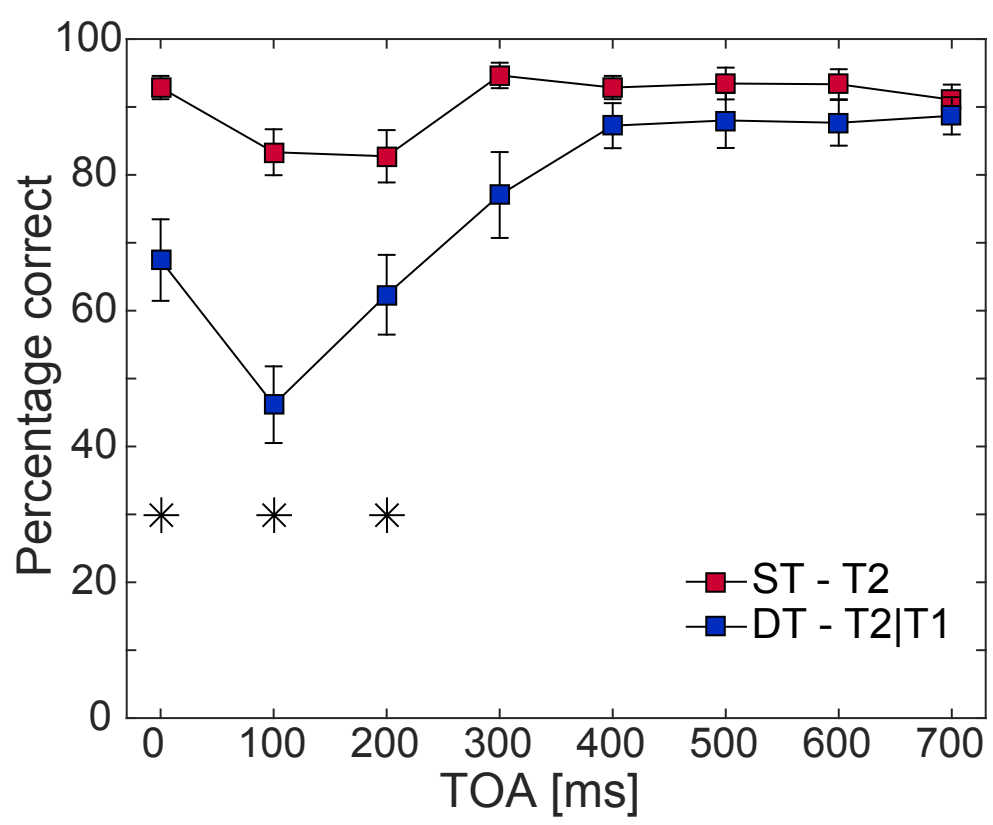

Figure 2.25: Performance in single- and dual-task. T2 performance dependent on TOA. The performance in the single-task is based on all trials, performance in the dual-task was calculated only for trials with correct T1 identification. Errorbars represent the SEM, asterisks mark TOAs with significant difference between singleand dual-task.

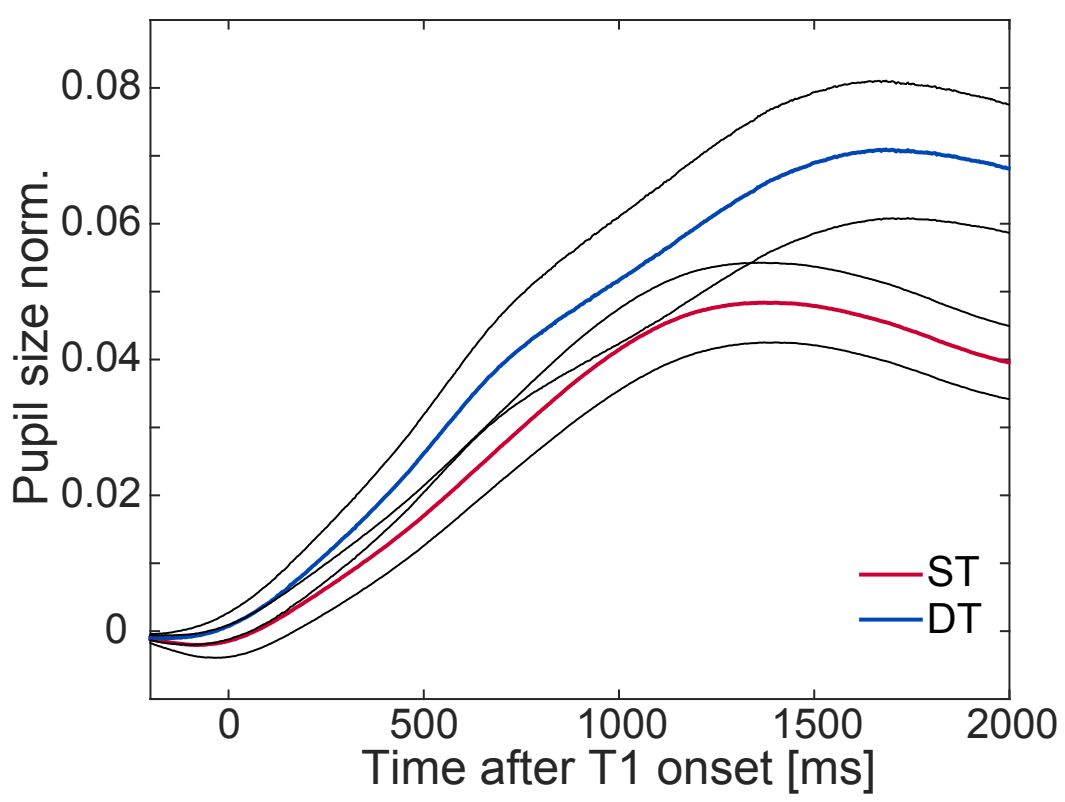

Figure 2.26: Normalized pupil size in single- and dual-task. Normalized pupil size in correct trials dependent on time relative to T1 onset. Blue and red curves show the mean values, the black curves represent the SEM. 
trials in the single-task. Figure 2.25 shows the performance for T2 identification in the single- and dual-task dependent on TOA. Performance was consistently high $(>80 \%)$ in the single-task for all TOAs. In the dual-task, performance was high for long TOAs (400-700ms, > 85\%), but dropped to a minimum of $46 \%$ for a TOA of $100 \mathrm{~ms}$. A two-way ANOVA (task x TOA) revealed a significant effect of task $(\mathrm{F}(1,91)=43.35, p<0.05)$, a significant effect of TOA $(\mathrm{F}(7,91)=23.92, p<0.05)$, and a significant interaction of both $(\mathrm{F}(7,91)=10.43, p<0.05)$. Post-hoc analysis revealed significant differences for TOAs of $0 \mathrm{~ms}$ to $300 \mathrm{~ms}$ ( $p<0.05$, paired t-test, FDR-corrected), but not for longer TOAs.

We next analyzed the pupil size change aligned to the onset of $\mathrm{T} 1$ occurrence based on trials with correct T2 identification (and additional correct T1 identification in the dual-task). Figure 2.26 shows that throughout the trial the mean pupil size was higher in the dual-task than in the single-task. The average pupil size in the period of 1000-2000ms differed significantly between single- and dual-task (mean ST: 0.045 $\pm 0.006(\mathrm{SEM})$, mean DT: 0.066 \pm 0.01 ; $p<0.01$, Wilcoxon signed-rank test). We used the deconvolution method to compute the attentional pulses underlying these pupil size changes over time. Figure 2.27 shows the strength of the attentional pulses in the singleand dual-task. For the dual-task, we found three clear peaks at 0ms, 500ms and $900 \mathrm{~ms}$. At $200 \mathrm{~ms}$, there was a minimum in the attentional strength. In contrast, in the single-task, the attentional strength rose at $0 \mathrm{~ms}$, and stayed at an elevated, but lower level than in the dual-task. The strength of attentional pulses differed between single- and dual-task for all timepoints except -200ms and 100ms after T1 onset (Wilcoxon rank sum test, $p<0.05$ ).

Figure 2.28 shows the attentional pulses computed separately for the different TOAs. In the single-tasks, we found that at T1 onset, there was an increase in the attentional pulse. Dependent on the TOA we found a second, higher pulse at a later time point which was roughly centered at the time point of T2 occurrence. For long TOAs, a third peak was located later in the trial. In the dual-task, we found two clear peaks with a similar strengths for all TOAs. The two peaks were all centered at $0 \mathrm{~ms}$ and at 400-600ms. Similar to the long TOAs in the single-task, a third peak occurred later in the trial.

We calculated the pupil size for blink trials for the TOAs that resulted in signif- 

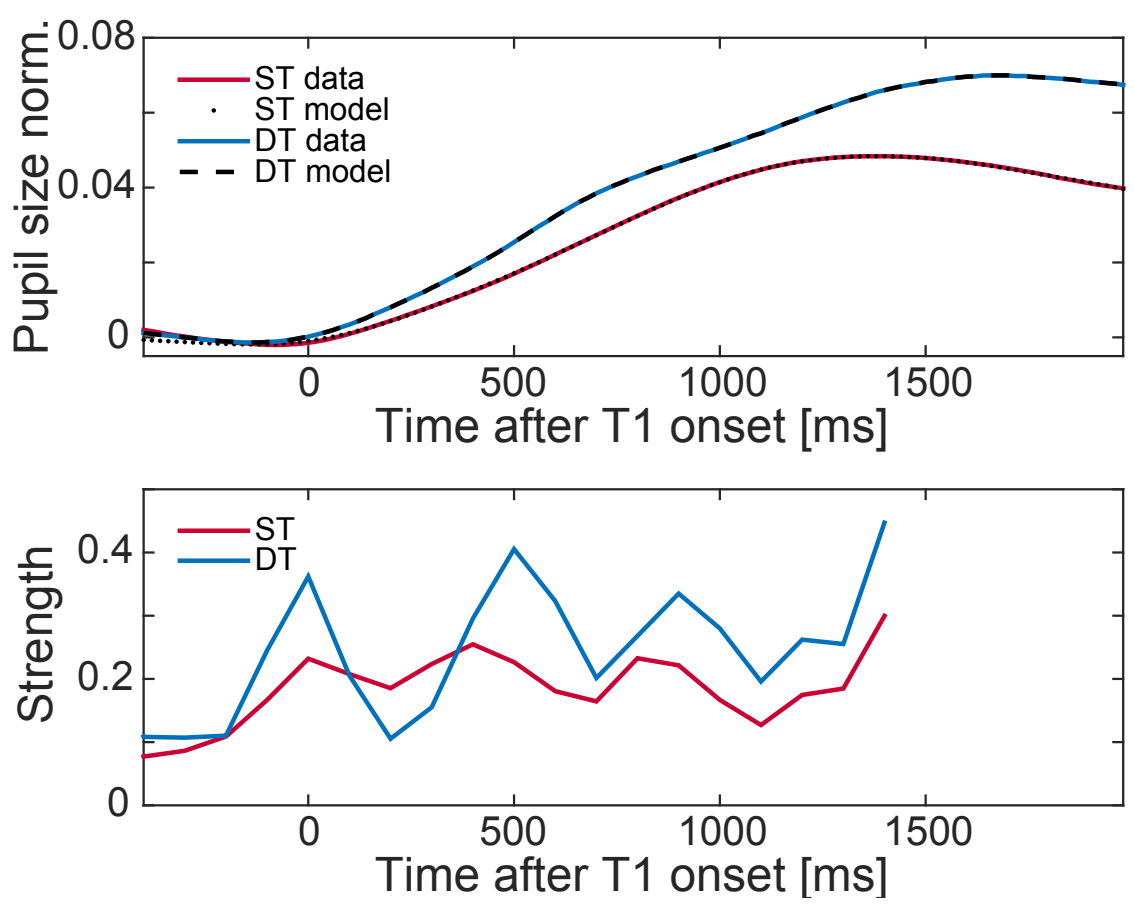

Figure 2.27: Deconvolution results correct trials in single- and dual-task. (A) Pupil size: model vs. data. (B) Strength of attentional pulses.

icant differences in performance. Figure 2.29 shows the pupil sizes dependent on time. We compared the mean pupil size in the interval of $1000-2000 \mathrm{~ms}$ for blink and no blink trials, and found that blink and no blink trials did not differ significantly (Wilcoxon signed rank test, $p=0.33$ ). For a TOA of $100 \mathrm{~ms}$, we applied the deconvolution method (figure 2.29). Whereas in the blink trials three distinct peaks were present, in the no blink trials we found only two peaks.

\section{Discussion}

We measured pupil dilation in a motion discrimination task that is similar to the design of attentional blink experiments. We wanted to test the hypothesis that LC activity is underlying the attentional blink by assuming that pupil dilation is an indirect measure for LC activity. We used a deconvolution method (Hoeks and Levelt, 1993; Wierda et al., 2012) to compute the underlying attentional pulses that supposedly lead to changes in pupil size.

The performance of the subjects in our task showed a similar, albeit a bit 

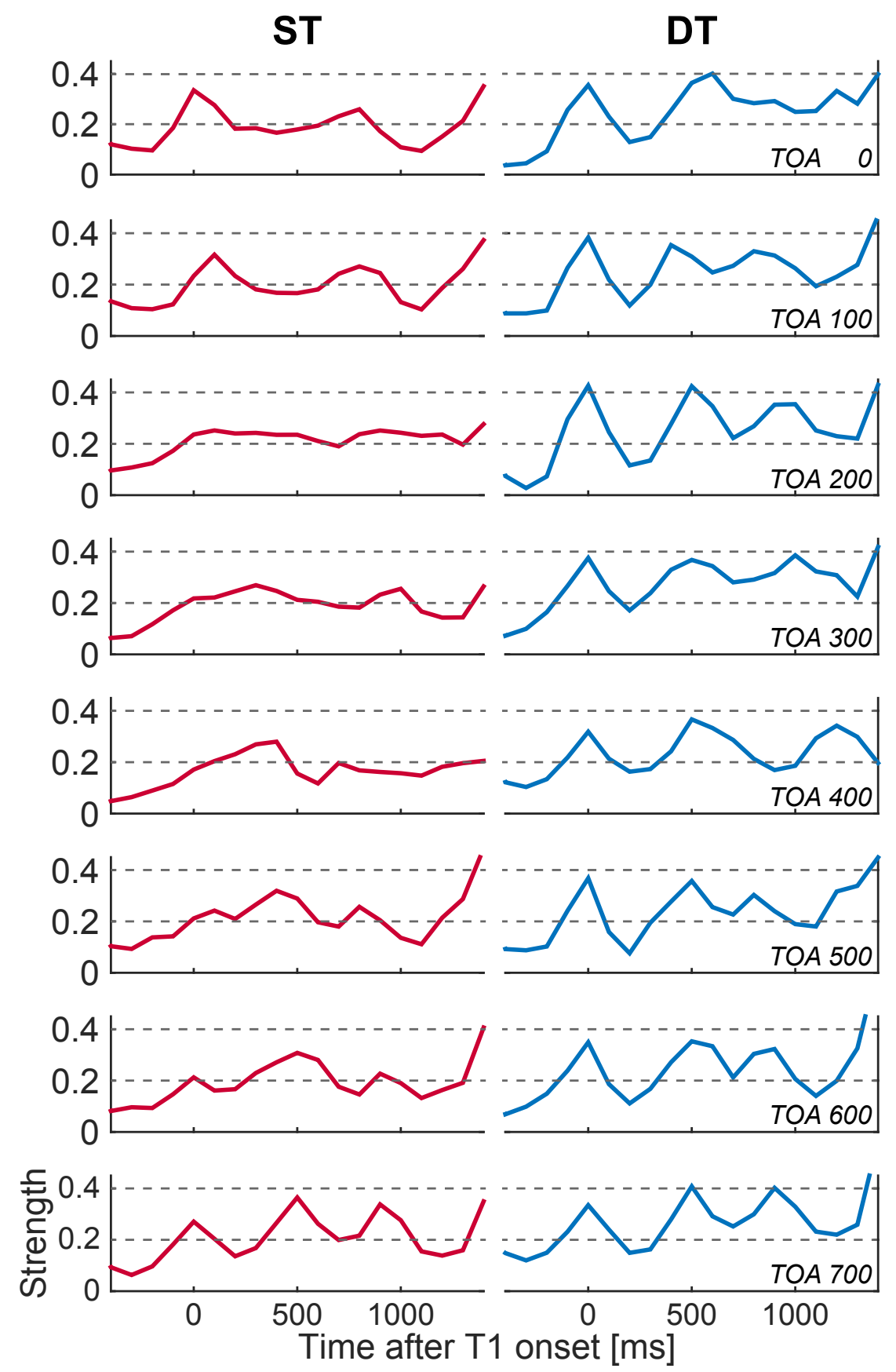

Figure 2.28: Deconvolution results. Strength of attentional pulses dependent on TOA for single- and dual-task. 

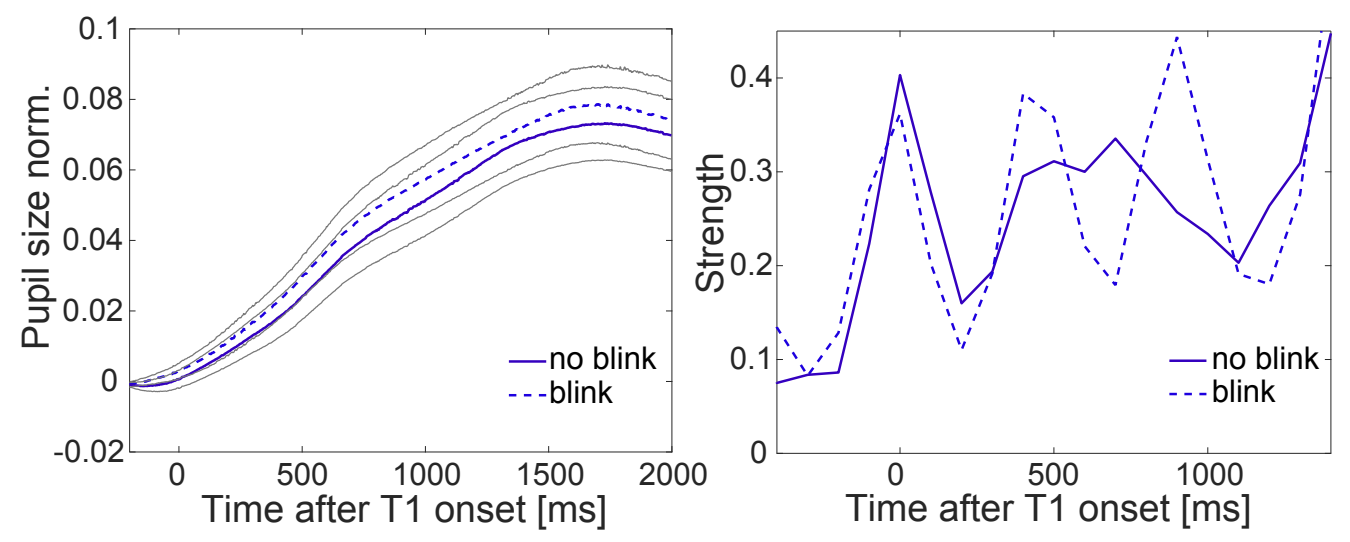

Figure 2.29: (A) Normalized pupil size in correct trials dependent on time relative to T1 onset for blink and no-blink trials. The bold and dashed blue curves show the mean values, the gray curves represent the SEM. (B) Deconvolution results. Strength of attentional pulses for blink and no-blink trials for a TOA of 100ms.

shorter, temporary drop as reported in previous attentional blink studies (e.g., Raymond et al., 1992). Our task differs from other attentional blink experiments in that our stimuli were not shown in the classical way of rapid serial visual presentation and are presented at two distinct spatial locations (similar to the paradigm used in Duncan et al., 1994). Nonetheless, performance was impaired in the dual-task for up to $300 \mathrm{~ms}$ after presentation of the first target stimulus T1. We used this experimental design, because we wanted to maintain the luminance of stimulation as constant as possible throughout the trial by using moving RDPs.

Pupil size was on average higher in the dual-task than in the single-task, which is in accordance with studies showing that pupil dilation depends on attentional load or on the number of target stimuli that need to be detected during a task (Zylberberg et al., 2012; Alnaes et al., 2014; Wahn et al., 2016). We found three distinct peaks in the underlying attentional pulses for the dualtask, while the pulses in the single-task were less distinct. In accordance with our hypothesis, we found that the attentional pulse related to $\mathrm{T} 1$ presentation was higher in the dual-task than in the single-task. Analyzing the pupil size separately for each TOA showed that in the single-task an attentional pulse was aligned to the onset of T2. In contrast, in the dual-task all TOAs showed the same temporal pattern: a first peak aligned to $\mathrm{T} 1$ onset and a second peak approximately 500ms later. These results support the hypothesis that 
in the dual-task, T2 does not evoke an attentional pulse when it is presented within the period of an attentional blink.

We found that for a TOA of $700 \mathrm{~ms}$, single-and dual-task resulted in the same pattern of attentional pulses. This is partly in accordance with our hypothesis that attentional pulses evoked by $\mathrm{T} 2$ presentation should not differ between single- and dual-task outside of the period of an attentional blink. However, for the single-task we would rather expect a single pulse aligned to $700 \mathrm{~ms}$.

Our results suggest that in the dual-task, $\mathrm{T} 1$ presentation triggered a sequence of attentional pulses influencing pupil size with a frequency of around 2-3Hz. This pattern was not changed by the presentation of T2. In the single-task in comparison, T1 presentation did not seem to trigger such a sequence; instead we found a small attentional pulse evoked by $\mathrm{T} 1$ and a second, higher peak aligned to the onset of T2. These findings support the hypothesis that the pupil size is affected by a phasic input being released in response to a target stimulus. The phasic input is followed by a period, in which no input is affecting the pupil size network. As described before, this pattern of activity can be found in the locus coeruleus, and therefore our results support the hypothesis that LC is involved during the attentional blink.

Contrary to previous studies (Zylberberg et al., 2012), we found no difference in overall pupil dilation between blink and no-blink trials. However, we found a difference in the temporal pattern of attentional pulses computed for a TOA of $100 \mathrm{~ms}$. While in the blink trials we find an oscillatory pattern with peaks at $0 \mathrm{~ms}, 450 \mathrm{~ms}$ and $900 \mathrm{~ms}$, in no-blink trials, there were only two peaks, one at $0 \mathrm{~ms}$ and a much wider peak centered at around 550ms. These findings suggest that there is an oscillatory input to the pupil network aligned to T1 onset and operating as long as subjects are still looking for a target stimulus. Such oscillatory activity in the delta range is usually linked to sleep states. However, there is evidence that delta oscillations also play a role during stimulus processing (e.g., Schroeder and Lakatos, 2009; Safaai et al., 2009). Safaai et al., for example, developed a model based on recordings in the LC and the somatosensory cortex of rats. The model proposes that LC activity and its coupling to the cortex can amplify delta oscillations in the cortex and during stimulus processing. However, if that was the case, and the input to the pupil network was also reflecting an input to the cortex influencing target 
detection, target detection should be easier for targets presented at a high of this oscillatory input. The oscillatory pattern that we find for the attentional pulses, would suggest that performance in the dual-task is higher for TOAs that fall onto the highs of the oscillation, i.e. 0ms, 450ms, $900 \mathrm{~ms}$ after T1 onset. However, this was neither the case in previous attentional blink studies nor in our experiment, as the performance is constantly high for long TOAs. Therefore, our results suggest that the source influencing the pupil size, be it the locus coeruleus or not, is not alone causing the processing deficit during an attentional blink. 



\section{Chapter 3}

\section{Summary and outlook}

This thesis contains four projects that investigated the complex nature of visual top-down attention from different perspectives and with different methods.

We provide anatomical evidence that the method of optogenetics can be used to target the fronto-visual and fronto-parietal network and its long-range axonal projections. Injection of viral vectors into frontal areas (FEF and PMv) resulted in profound opsin expression around the injection location. We confirmed that the opsins are not only incorporated into the membrane of the somata in the injection region, but are present within the dendrites and axons of the neurons. We find opsin-positive axons in the white matter and in distant target areas, like the parietal area MIP and the visual area MT. We show that 10 weeks after viral vector injection, opsins can already be found in a decent amount in the axons in distant areas. Our results indicate that optical stimulation of axonal projections can be conducted in the fronto-visual and fronto-parietal network.

Based on these results, we injected one of the previously tested viral vectors into the FEF of two animals trained in a spatial attention task. We inhibited the connection of the FEF to visual area MT in one monkey while the animal was conducting a visual spatial attention task. Based on numerous evidence, the FEF has been suggested to play a fundamental role in guiding visual attention. However, these studies were unable to distinguish between direct effects of the FEF on extrastriate visual areas and indirect effects. By using optogenetics, we show that the modulation that the FEF exerts on visual area MT during attention results at least partly from a direct input from the FEF. 
In our experiments, attentional enhancement of firing rates was reduced by up to $31 \%$ when the input from the FEF was inhibited or reduced by laser stimulation. Our results show that attentional modulation in an area of the dorsal visual pathway seems to be influenced by the FEF similar to areas of the ventral visual pathway.

However, our results and the results of other studies (Gregoriou et al., 2014) indicate that the FEF might not be the only source of attentional signals in visual areas. It is known that several other cortical areas, for example, area LIP, and subcortical areas, like the superior colliculus, play a role in top-down attention (Bisley and Goldberg, 2010; Krauzlis et al., 2013). In addition, the locus coeruleus, the nucleus basalis of Meynert or the ventral tegmental area - areas that send noradrenergic, cholinergic or dopaminergic projections to widespread brain areas - most likely play a role during top-down attention (Noudoost and Moore, 2011b).

In the human subject, we investigated the phenomenon of the attentional blink in two further studies. Since an attentional blink has primarily been shown to occur for stimuli processed in the ventral visual pathway, we asked whether it also occurs for stimuli processed in the dorsal visual pathway. We show that motion stimuli do evoke an attentional blink in a similar way as do stationary visual stimuli. In addition, we found that an attentional blink caused by one type of stimulus also affects processing of the other kind of stimulus. These results suggest that an attentional blink affects the whole visual processing system. In line with the results of several previous and our physiology study (i.e., that the FEF is involved in attentional modulation within dorsal and ventral stream visual areas) the findings speak for the existence of a unified attentional system operating comprehensively within the brain.

We used a similar, but simplified experimental design in a follow-up psychophysics study. In addition to behavioral performance, we measured pupil size of the subjects. It is known that cognitive processes, e.g., attention, affect the pupil size. A model had been derived that allows to compute attentional pulses underlying pupil size changes (Hoeks and Levelt, 1993; Wierda et al., 2012). We used this model to analyze pupil size, relate it to performance, and create a link to the activity of the locus coeruleus. The locus coeruleus has 
been suggested to influence pupil size and to play a role in causing an attentional blink. We find evidence for the hypothesis that the attentional blink and locus coeruleus activity might be related. Our results show that the pupil size is differently affected by the single-and dual-tasks. While we found an attentional pulse aligned to the onset of the second target in the single-task, we found no attentional pulse aligned to the second target in the dual-task. Instead, we found a pattern of pulses aligned only to the onset of the first target. Our results are in line with the hypothesis that the locus coeruleus is involved in the phenomenon of the attentional blink. However, we did not find evidence that the pupil size reflects the behavioral outcome of the trials.

Top-down attention is a complex process that involves a complex network of brain areas and neurotransmitters. It can neither be reduced to the interaction of two brain areas, nor can it be understood by measuring psychophysical performance. Understanding top-down attention necessitates the disentangling of the network interactions including cortical areas, but also subcortical areas, and maybe even the whole body. Our projects explored two different mechanisms of attentional modulation: a modulation by the cortical prefrontal cortex and a modulation by a subcortical structure that acts by releasing the neuromodulator noradrenaline into multiple target areas. As mentioned in the introduction, the most prescribed drugs for treatment of ADHD act on the noradrenaline and dopamine signaling. One of the emerging questions out of this and our results is whether these neurotransmitters also play a role during attention in visual areas and whether and how the release of these neurotransmitters interacts with the input and the modulation by the prefrontal cortex (and maybe also the parietal cortex). Since visual area MT receives a direct input from the locus coeruleus (Tigges et al., 1982), it is tempting to ask for the role of this input during top-down attention and whether there might be an interaction with the modulation by the FEF.

Investigating the influence of two areas onto a third area had been very difficult in the past. However, combining pathway-specific optogenetics with neuropharmacological manipulations or cell-type specific optogenetics provides a new possibility to understand the interaction of cortical and subcortical structures during top-down attention. 
If we want to understand psychological disorders like ADHD, we have to study the complex attentional networks and the interactions of cortical and subcortical areas. 


\section{Bibliography}

L. Acker, E. N. Pino, E. S. Boyden, and R. Desimone. FEF inactivation with improved optogenetic methods. Proceedings of the National Academy of Sciences, 113(46):E7297-E7306, 2016.

G. K. Aghajanian, J. M. Cedarbaum, and R. Y. Wang. Evidence for norepinephrine-mediated collateral inhibition of locus coeruleus neurons. Brain Research, 136(3):570-577, 1977.

T. D. Albright. Direction and orientation selectivity of neurons in visual area MT of the macaque. Journal of Neurophysiology, 52(6):1106-1130, 1984.

T. D. Albright and R. Desimone. Local precision of visuotopic organization in the middle temporal area (MT) of the macaque. Experimental Brain Research, 65(3):582-592, 1987.

T. D. Albright, R. Desimone, and C. G. Gross. Columnar organization of directionally selective cells in visual area MT of the macaque. Journal of Neurophysiology, 51(1):16-31, 1984.

John M. Allman and Jon H. Kaas. A representation of the visual field in the caudal third of the middle temporal gyrus of the owl monkey (aotus trivirgatus). Brain Research, 31(1):85-105, 1971.

D. Alnaes, M. H. Sneve, T. Espeseth, T. Endestad, S. H. P. van de Pavert, and B. Laeng. Pupil size signals mental effort deployed during multiple object tracking and predicts brain activity in the dorsal attention network and the locus coeruleus. Journal of Vision, 14(4):1, 2014. 
J. C. Anderson, H. Kennedy, and K. A. C. Martin. Pathways of attention: Synaptic relationships of frontal eye field to V4, lateral intraparietal cortex, and area 46 in macaque monkey. Journal of Neuroscience, 31(30):1087210881, 2011.

K. Anton-Erxleben, V. M. Stephan, and S. Treue. Attention reshapes centersurround receptive field structure in macaque cortical area MT. Cerebral Cortex, 19(10):2466-2478, 2009.

K. M. Armstrong and T. Moore. Rapid enhancement of visual cortical response discriminability by microstimulation of the frontal eye field. Proceedings of the National Academy of Sciences, 104(22):9499-9504, 2007.

A. F. T. Arnsten. Through the looking glass: Differential noradenergic modulation of prefrontal cortical function. Neural Plasticity, 7(1-2):133-46, 2000.

A. F. T. Arnsten and P. S. Goldman-Rakic. Selective prefrontal cortical projections to the region of the locus coeruleus and raphe nuclei in the rhesus monkey. Brain Research, 306(1-2):9-18, 1984.

D. F. Aschauer, S. Kreuz, and S. Rumpel. Analysis of transduction efficiency, tropism and axonal transport of AAV serotypes 1, 2, 5, 6, 8 and 9 in the mouse brain. PLoS ONE, 8(9):e76310, 2013.

S. V. Astafiev, G. L. Shulman, C. M. Stanley, A. Z. Snyder, D. C. Van Essen, and M. Corbetta. Functional organization of human intraparietal and frontal cortex for attending, looking, and pointing. Journal of Neuroscience, 23(11):4689-4699, 2003.

G. Aston-Jones and F. E. Bloom. Norepinephrine-containing locus coeruleus neurons in behaving rats exhibit pronounced responses to non-noxious environmental stimuli. Journal of Neuroscience, 1(8):887-900, 1981a.

G. Aston-Jones and F. E. Bloom. Activity of norepinephrine-containing locus coeruleus neurons in behaving rats anticipates fluctuations in the sleepwaking cycle. Journal of Neuroscience, 1(8):876-886, 1981 b. 
G. Aston-Jones and J. D. Cohen. An integrative theory of locus coeruleusnorepinephrine function: Adaptive gain and optimal performance. Annual Review of Neuroscience, 28(1):403-450, 2005.

G. Aston-Jones, M. Ennis, V. A. Pieribone, W. T. Nickell, and M. T. Shipley. The brain nucleus locus coeruleus: restricted afferent control of a broad efferent network. Science, 234(4777):734-737, 1986.

G. Aston-Jones, J. Rajkowski, P. Kubiak, and T. Alexinsky. Locus coeruleus neurons in monkey are selectively activated by attended cues in a vigilance task. Journal of Neuroscience, 14(7):4467-4480, 1994.

S. Bakola, L. Passarelli, T. Huynh, D. Impieri, K. H. Worthy, P. Fattori, C. Galletti, K. J. Burman, and M. G. P. Rosa. Cortical afferents and myeloarchitecture distinguish the medial intraparietal area (mip) from neighboring subdivisions of the macaque cortex. eNeuro, 4(6), 2017.

P. Barone, A. Batardiere, K. Knoblauch, and H. Kennedy. Laminar distribution of neurons in extrastriate areas projecting to visual areas V1 and V4 correlates with the hierarchical rank and indicates the operation of a distance rule. Journal of Neuroscience, 20(9):3263-3281, 2000.

J. Beatty. Task-evoked pupillary responses, processing load, and the structure of processing resources. Psychological Bulletin, 91(2):276-292, 1982.

C. E. Beevor and V. Horsley. IX. A further minute analysis by electric stimulation of the so-called motor region of cortex cerebri in the monkey (macacus sinicus). Philosophical Transactions of the Royal Society of London, 179: 205-256, 1888.

D. L. Benson, P. J. Isackson, S. H. Hendry, and E. G. Jones. Differential gene expression for glutamic acid decarboxylase and type II calcium-calmodulindependent protein kinase in basal ganglia, thalamus, and hypothalamus of the monkey. Journal of Neuroscience, 11(6):1540-1564, 1991.

D. L. Benson, P. J. Isackson, C. M. Gall, and E. G. Jones. Contrasting patterns in the localization of glutamic acid decarboxylase and $\mathrm{Ca}^{2+} /$ calmodulin protein kinase gene expression in the rat central nervous system. Neuroscience, 46(4):825-849, 1992. 
C. W. Berridge and B. D. Waterhouse. The locus coeruleus-noradrenergic system: Modulation of behavioral state and state-dependent cognitive processes. 42(1):33-84, 2003.

N. P. Bichot, J. D. Schall, and K. G. Thompson. Visual feature selectivity in frontal eye fields induced by experience in mature macaques. Nature, 381 (6584):697-699, 1996.

P. Binda, M. Pereverzeva, and S. O. Murray. Attention to bright surfaces enhances the pupillary light reflex. Journal of Neuroscience, 33(5):2199$2204,2013$.

P. Binda, M. Pereverzeva, and S. O. Murray. Pupil size reflects the focus of feature-based attention. Journal of Neurophysiology, 112(12):3046-3052, 2014.

J. W. Bisley and M. E. Goldberg. Attention, intention, and priority in the parietal lobe. Annual Review of Neuroscience, 33:1-21, 2010.

J. Botella and C. W. Eriksen. Filtering versus parallel processing in RSVP tasks. Perception \& Psychophysics, 51(4):334-343, 1992.

E. S. Boyden, F. Zhang, E. Bamberg, G. Nagel, and K. Deisseroth. Millisecond-timescale, genetically targeted optical control of neural activity. Nature Neuroscience, 8(9):1263-1268, 2005.

D. C. Bradley, N. Qian, and R. A. Andersen. Integration of motion and stereopsis in middle temporal cortical area of macaques. Nature, 373(6515): 609-611, 1995.

L. Briars and T. Todd. A review of pharmacological management of attentiondeficit hyperactivity disorder. The Journal of Pediatric Pharmacology and Therapeutics, 21(3):192-206, 2016.

K. H. Britten, W. T. Newsome, M. N. Shadlen, S. Celebrini, and J. A. Movshon. A relationship between behavioral choice and the visual responses of neurons in macaque MT. Visual Neuroscience, 13(1):87-100, 1996. 
K. Brodmann. Vergleichende Lokalisationslehre der Grosshirnrinde in ihren Prinzipien dargestellt auf Grund des Zellenbaues. Barth, Leipzig, 1909.

C. J. Bruce and M. E. Goldberg. Primate frontal eye fields. I. Single neurons discharging before saccades. Journal of Neurophysiology, 53(3):603-635, 1985 .

C. J. Bruce, M. E. Goldberg, M. C. Bushnell, and G. B. Stanton. Primate frontal eye fields. II. Physiological and anatomical correlates of electrically evoked eye movements. Journal of Neurophysiology, 54(3):714-734, 1985.

J. Bullier, J. D. Schall, and A. Morel. Functional streams in occipito-frontal connections in the monkey. 76(1-2):89-97, 1996.

C. Burger, O. S. Gorbatyuk, M. J. Velardo, C. S. Peden, P. Williams, S. Zolotukhin, P. J. Reier, R. J. Mandel, and N. Muzyczka. Recombinant AAV viral vectors pseudotyped with viral capsids from serotypes 1, 2, and 5 display differential efficiency and cell tropism after delivery to different regions of the central nervous system. Molecular Therapy, 10(2):302-317, 2004.

A. Burgos-Robles, E. Y. Kimchi, E. M. Izadmehr, M. J. Porzenheim, W. A. Ramos-Guasp, E. H. Nieh, A. C. Felix-Ortiz, P. Namburi, C. A. Leppla, K. N. Presbrey, K. K. Anandalingam, P. A. Pagan-Rivera, M. Anahtar, A. Beyeler, and K. M. Tye. Amygdala inputs to prefrontal cortex guide behavior amid conflicting cues of reward and punishment. Nature Neuroscience, 20(6):824-835, 2017.

G. Bush. Attention-deficit hyperactivity disorder and attention networks. Neuropsychopharmacology, 35(1):278-300, 2010.

F. W. Campbell and A. H. Gregory. Effect of size of pupil on visual acuity. Nature, 187(4743):1121-1123, 1960.

F. X. Castellanos and E. Proal. Large-scale brain systems in ADHD: Beyond the prefrontal-striatal model. Trends in Cognitive Sciences, 16(1):17-26, 2012 .

M. J. Castle, Z. T. Gershenson, A. R. Giles, E. L. F. Holzbaur, and J. H. Wolfe. Adeno-associated virus serotypes 1, 8, and 9 share conserved mechanisms 
for anterograde and retrograde axonal transport. Human Gene Therapy, 25 (8):705-720, 2014.

J. Cavanaugh, W. M. Joiner, and R. H. Wurtz. Suppressive surrounds of receptive fields in monkey frontal eye field. Journal of Neuroscience, 32 (35):12284-12293, 2012.

M. M. Chun and M. C. Potter. A two-stage model for multiple target detection in rapid serial visual presentation. Journal of Experimental Psychology: Human Perception and Performance, 21(1):109-127, 1995.

K. L. Clark and B. Noudoost. The role of prefrontal catecholamines in attention and working memory. Frontiers in Neural Circuits, 8:8, 2014.

L. L. Cloutman. Interaction between dorsal and ventral processing streams: Where, when and how? Brain and Language, 127(2):251-263, 2013.

J. Y. Cohen, P. Pouget, R. P. Heitz, G. F. Woodman, and J. D. Schall. Biophysical support for functionally distinct cell types in the frontal eye field. Journal of Neurophysiology, 101(2):912-916, 2009.

E. P. Cook and J. H. R. Maunsell. Attentional modulation of behavioral performance and neuronal responses in middle temporal and ventral intraparietal areas of macaque monkey. Journal of Neuroscience, 22(5):1994-2004, 2002.

M. Corbetta. Frontoparietal cortical networks for directing attention and the eye to visual locations: Identical, independent, or overlapping neural systems? Proceedings of the National Academy of Sciences, 95(3):831-838, 1998.

M. Corbetta, E. Akbudak, T. E. Conturo, A. Z. Snyder, J. M. Ollinger, H. A. Drury, M. R. Linenweber, S. E. Petersen, M. E. Raichle, D. C. Van Essen, and G. L. Shulman. A common network of functional areas for attention and eye movements. Neuron, 21(4):761-773, 1998.

J. T. Coull, A. C. Nobre, and C. D. Frith. The noradrenergic alpha2 agonist clonidine modulates behavioral and neuroanatomical correlates of human attentional orienting and alerting. Cerebral Cortex, 11(1):73-84, 2001. 
B. G. Cragg. The topography of the afferent projections in the circumstriate visual cortex of the monkey studied by the nauta method. Vision Research, $9(7): 733-747,1969$.

L. Craighero and G. Rizzolatti. Neurobiology of Attention. The premotor theory of attention. Academic Press, 2005.

B. De Martino, B. A. Strange, and R. J. Dolan. Noradrenergic neuromodulation of human attention for emotional and neutral stimuli. Psychopharmacology, 197(1):127-136, 2008.

G. C. DeAngelis and W. T. Newsome. Organization of disparity-selective neurons in macaque area MT. Journal of Neuroscience, 19(4):1398-1415, 1999.

J. DeFelipe and I. Fariñas. The pyramidal neuron of the cerebral cortex: Morphological and chemical characteristics of the synaptic inputs. Progress in Neurobiology, 39(6):563-607, 1992.

R. Desimone and L. G. Ungerleider. Multiple visual areas in the caudal superior temporal sulcus of the macaque. Journal of Comparative Neurology, 248(2):164-189, 1986.

V. Di Lollo, J. I. Kawahara, S. M. Shahab Ghorashi, and James T. Enns. The attentional blink: Resource depletion or temporary loss of control? Psychological Research, 69(3):191-200, 2005.

G. Di Pasquale, B. L. Davidson, C. S. Stein, I. Martins, D. Scudiero, A. Monks, and J. A. Chiorini. Identification of PDGFR as a receptor for AAV-5 transduction. Nature Medicine, 9(10):1306-1312, 2003.

I. Diester, M. T. Kaufman, M. Mogri, R. Pashaie, W. Goo, O. Yizhar, C. Ramakrishnan, K. Deisseroth, and K. V. Shenoy. An optogenetic toolbox designed for primates. Nature Neuroscience, 14(3):387-397, 2011.

R. Dubner and S. M. Zeki. Response properties and receptive fields of cells in an anatomically defined region of the superior temporal sulcus in the monkey. Brain Research, 35(2):528-532, 1971. 
J. Duncan, R. Ward, and K. Shapiro. Direct measurement of attentional dwell time in human vision. Nature, 369(6478):313-315, 1994.

P. E. Dux and R. Marois. How humans search for targets through time: A review of data and theory from the attentional blink. Attention, perception E psychophysics, 71(8):1683-1700, 2010.

T. M. Egan, G. Henderson, R. A. North, and J. T. Williams. Noradrenalinemediated synaptic inhibition in rat locus coeruleus neurones. The Journal of Physiology, 345:477-488, 1983.

D. J. Felleman and J. H. Kaas. Receptive-field properties of neurons in middle temporal visual area (MT) of owl monkeys. Journal of Neurophysiology, 52 (3):488-513, 1984.

D. J. Felleman and D. C. Van Essen. Distributed hierarchical processing in the primate cerebral cortex. Cerebral Cortex, 1(1):1-47, 1991.

V. P. Ferrera, T. A. Nealey, and J. H. Maunsell. Responses in macaque visual area V4 following inactivation of the parvocellular and magnocellular LGN pathways. Journal of Neuroscience, 14(4):2080-2088, 1994.

D. Ferrier. Experiments on the brain of monkeys. - No. I. Proceedings of the Royal Society of London, 23:409-430, 1874.

S. L. Foote, G. Aston-Jones, and F. E. Bloom. Impulse activity of locus coeruleus neurons in awake rats and monkeys is a function of sensory stimulation and arousal. Proceedings of the National Academy of Sciences, 77 (5):3033-3037, 1980.

R. Freedman, S. L. Foote, and F. E. Bloom. Histochemical characterization of a neocortical projection of the nucleus locus coeruleus in the squirrel monkey. Journal of Comparative Neurology, 164(2):209-231, 1975.

A. Galvan, X. Hu, Y. Smith, and T. Wichmann. Effects of optogenetic activation of corticothalamic terminals in the motor thalamus of awake monkeys. Journal of Neuroscience, 36(12):3519-3530, 2016. 
A. Galvan, W. R. Stauffer, L. Acker, Y. El-Shamayleh, K.-I. Inoue, S. Ohayon, and M. C. Schmid. Nonhuman primate optogenetics: Recent advances and future directions. Journal of Neuroscience, 37(45):10894-10903, 2017.

D. L. Garver and J. R. Sladek. Monoamine distribution in primate brain. I. Catecholamine-containing perikarya in the brain stem of macaca speciosa. Journal of Comparative Neurology, 159(3):289-304, 1975.

R. Gattass and C. G. Gross. Visual topography of striate projection zone (MT) in posterior superior temporal sulcus of the macaque. Journal of Neurophysiology, 46(3):621-638, 1981.

A. Gerits, R. Farivar, B. R. Rosen, L. L. Wald, E. S. Boyden, and W. Vanduffel. Optogenetically induced behavioral and functional network changes in primates. Current Biology, 22(18):1722-1726, 2012.

G. M. Ghose and J. H. R. Maunsell. Attentional modulation in visual cortex depends on task timing. Nature, 419(6907):616-620, 2002.

M. S. Gilzenrat, S. Nieuwenhuis, M. Jepma, and J. D. Cohen. Pupil diameter tracks changes in control state predicted by the adaptive gain theory of locus coeruleus function. Cognitive, Affective, \& Behavioral Neuroscience, 10(2):252-269, 2010.

M. E. Goldberg and M. C. Bushnell. Behavioral enhancement of visual responses in monkey cerebral cortex. II. Modulation in frontal eye fields specifically related to saccades. Journal of Neurophysiology, 46(4):773-787, 1981.

M. A. Goodale and A. D. Milner. Separate visual pathways for perception and action. Trends in Neurosciences, 15(1):20-25, 1992.

V. Gradinaru, F. Zhang, C. Ramakrishnan, J. Mattis, R. Prakash, I. Diester, I. Goshen, K. R. Thompson, and K. Deisseroth. Molecular and cellular approaches for diversifying and extending optogenetics. Cell, 141(1):154$165,2010$.

T. D. Grandison, T. G. Ghirardelli, and H. E. Egeth. Beyond similarity: Masking of the target is sufficient to cause the attentional blink. Perception \& Psychophysics, 59(2):266-274, 1997. 
G. G. Gregoriou, S. J. Gotts, H. Zhou, and R. Desimone. High-frequency, long-range coupling between prefrontal and visual cortex during attention. Science, 324(5931):1207-1210, 2009.

G. G. Gregoriou, S. J. Gotts, and R. Desimone. Cell-type-specific synchronization of neural activity in FEF with V4 during attention. Neuron, 73(3): 581-594, 2012.

G. G. Gregoriou, A. F. Rossi, L. G. Ungerleider, and R. Desimone. Lesions of prefrontal cortex reduce attentional modulation of neuronal responses and synchrony in V4. Nature Neuroscience, 17(7):1003-1011, 2014.

K. V. Haak and C. F. Beckmann. Objective analysis of the topological organization of the human cortical visual connectome suggests three visual pathways. Cortex, 2017. doi: 10.1016/j.cortex.2017.03.020.

R. Halliday, E. Callaway, and R. Lannon. The effects of clonidine and yohimbine on human information processing. Psychopharmacology, 99(4):563-566, 1989.

F. H. Hamker. The reentry hypothesis: The putative interaction of the frontal eye field, ventrolateral prefrontal cortex, and areas V4, IT for attention and eye movement. Cerebral Cortex, 15(4):431-447, 2005.

X. Han, X. Qian, J. G. Bernstein, H. Zhou, G. T. Franzesi, P. Stern, R. T. Bronson, A. M. Graybiel, R. Desimone, and E. S. Boyden. Millisecondtimescale optical control of neural dynamics in the nonhuman primate brain. Neuron, 62(2):191-198, 2009.

X. Han, B. Y. Chow, H. Zhou, N. C. Klapoetke, A. Chuong, R. Rajimehr, A. Yang, M. V. Baratta, J. Winkle, R. Desimone, and E. S. Boyden. A high-light sensitivity optical neural silencer: Development and application to optogenetic control of non-human primate cortex. Frontiers in Systems Neuroscience, 5:18, 2011.

J. Herrero, M. Roberts, L. Delicato, M. Gieselmann, P. Dayan, and A. Thiele. Acetylcholine contributes through muscarinic receptors to attentional modulation in v1. Nature, 454(7208):1110-1114, 2008. 
T. M. Herrington and J. A. Assad. Neural activity in the middle temporal area and lateral intraparietal area during enogenously cued shifts of attention. Journal of Neuroscience, 29(45):14160-14176, 2009.

E. H. Hess and J. M. Polt. Pupil size in relation to mental activity during simple problem-solving. Science, 143(3611):1190-1192, 1964.

M. H. Histed, A. M. Ni, and J. H. R. Maunsell. Insights into cortical mechanisms of behavior from microstimulation experiments. Progress in Neurobiology, 103:115-130, 2013.

B. Hoeks and W. J. M. Levelt. Pupillary dilation as a measure of attention: A quantitative system analysis. Behavior Research Methods, Instruments, $\&$ Computers, 25(1):16-26, 1993.

M. F. Huerta, L. A. Krubitzer, and J. H. Kaas. Frontal eye field as defined by intracortical microstimulation in squirrel monkeys, owl monkeys, and macaque monkeys II. Cortical connections. Journal of Comparative Neurology, 265(3):332-361, 1987.

G. Ibos and D. J. Freedman. Interaction between spatial and feature attention in posterior parietal cortex. Neuron, 91(4):931-941, 2016.

K.-I. Inoue, M. Takada, and M. Matsumoto. Neuronal and behavioural modulations by pathway-selective optogenetic stimulation of the primate oculomotor system. Nature Communications, 6(8378), 2015.

Y. Izawa and H. Suzuki. Activity of fixation neurons in the monkey frontal eye field during smooth pursuit eye movements. Journal of Neurophysiology, 112(2):249-262, 2014.

Y. Izawa, H. Suzuki, and Y. Shinoda. Response properties of fixation neurons and their location in the frontal eye field in the monkey. Journal of Neurophysiology, 102(4):2410-2422, 2009.

W. James. The Principles of Psychology (Vols. 1 \& 2). New York Holt, 1890.

E. Jodo, C. Chiang, and G. Aston-Jones. Potent excitatory influence of prefrontal cortex activity on noradrenergic locus coeruleus neurons. Neuroscience, 83(1):63-79, 1998. 
E. G. Jones, G. W. Huntley, and D. L. Benson. Alpha calcium/calmodulindependent protein kinase II selectively expressed in a subpopulation of excitatory neurons in monkey sensory-motor cortex: comparison with GAD-67 expression. Journal of Neuroscience, 14(2):611-629, 1994.

J. S. Joseph, M. M. Chun, and K. Nakayama. Attentional requirements in a 'preattentive' feature search task. Nature, 387(6635):805-807, 1997.

S. Joshi, Y. Li, R. M. Kalwani, and J. I. Gold. Relationships between pupil diameter and neuronal activity in the locus coeruleus, colliculi, and cingulate cortex. Neuron, 89(1):221-234, 2016.

N. Kaludov, K. E. Brown, R. W. Walters, J. Zabner, and J. A. Chiorini. Adeno-associated virus serotype 4 (AAV4) and AAV5 both require sialic acid binding for hemagglutination and efcient transduction but differ in sialic acid linkage specicity. Journal of Virology, 75(15):6884-6893, 2001.

S. Katzner, L. Busse, and S. Treue. Attention to the color of a moving stimulus modulates motion-signal processing in macaque area MT: evidence for a unified attentional system. Frontiers in Systems Neuroscience, 3:12, 2009.

J.-I. Kawahara, V. Di Lollo, and J. T. Enns. Attentional requirements in visual detection and identification: Evidence from the attentional blink. Journal of Experimental Psychology: Human Perception and Performance, 27(4):969-984, 2001.

F. J. Kellie and K. L. Shapiro. Object file continuity predicts attentional blink magnitude. Perception \& Psychophysics, 66(4):692-712, 2004.

P. S. Khayat, A. Pooresmaeili, and P. R. Roelfsema. Time course of attentional modulation in the frontal eye field during curve tracing. Journal of Neurophysiology, 101(4):1813-1822, 2009.

K. Kihara, T. Takeuchi, S. Yoshimoto, H. M. Kondo, and J. I. Kawahara. Pupillometric evidence for the locus coeruleus-noradrenaline system facilitating attentional processing of action-triggered visual stimuli. Frontiers in Psychology, 6:827, 2015. 
S. Y. Kim, A. Adhikari, S. Y. Lee, J. H. Marshel, C. K. Kim, C. S. Mallory, M. Lo, S. Pak, J. Mattis, B. K. Lim, R. C. Malenka, M. R. Warden, R. Neve, K. M. Tye, and K. Deisseroth. Diverging neural pathways assemble a behavioural state from separable features in anxiety. Nature, 496(7444): 219-223, 2013.

Y. Kodaka, A. Mikami, and K. Kubota. Neuronal activity in the frontal eye field of the monkey is modulated while attention is focused on to a stimulus in the peripheral visual field, irrespective of eye movement. Neuroscience Research, 28(4):291-298, 1997.

R. J. Krauzlis, L. P. Lovejoy, and L. P. Zenon. Superior colliculus and visual spatial attention. Annual Review of Neuroscience, 36:165-182, 2013.

B. Laeng and T. Endestad. Bright illusions reduce the eye's pupil. Proceedings of the National Academy of Sciences, 109(6):2162-2167, 2012.

L. Lagae, S. Raiguel, and G. A. Orban. Speed and direction selectivity of macaque middle temporal neurons. Journal of Neurophysiology, 69(1):1939, 1993.

D. H. Lawrence. Two studies of visual search for word targets with controlled rates of presentation. Perception \& Psychophysics, 10(2):85-89, 1971.

A. T. Lee, D. Vogt, J. L. Rubenstein, and V. S. Sohal. A class of GABAergic neurons in the prefrontal cortex sends long-range projections to the nucleus accumbens and elicits acute avoidance behavior. Journal of Neuroscience, 34(35):11519-11525, 2014.

J. Lee and J. H. R. Maunsell. Attentional modulation of MT neurons with single or multiple stimuli in their receptive fields. Journal of Neuroscience, 30(8):3058-3066, 2010.

J. Lee and J. H.R. Maunsell. A normalization model of attentional modulation of single unit responses. PLoS ONE, 4(2):e4651, 2009.

S. J. Lehmann and B. D. Corneil. Transient pupil dilation after subsaccadic microstimulation of primate frontal eye fields. Journal of Neuroscience, 36 (13):3765-3776, 2016. 
G. R. Leichnetz. Inferior frontal eye field projections to the pursuit-related dorsolateral pontine nucleus and middle temporal area (MT) in the monkey. Visual Neuroscience, 3(2):171-80, 1989.

P. Levitt, P. Rakic, and P. Goldman-Rakic. Region-specific distribution of catecholamine afferents in primate cerebral cortex: A fluorescence histochemical analysis. Journal of Comparative Neurology, 227(1):23-36, 1984.

X. B. Liu and E. G. Jones. Localization of alpha type II calcium calmodulindependent protein kinase at glutamatergic but not gamma-aminobutyric acid (GABAergic) synapses in thalamus and cerebral cortex. Proceedings of the National Academy of Sciences, 93(14):7332-7336, 1996.

E. J. Livesey and I. M. Harris. Target sparing effects in the attentional blink depend on type of stimulus. Attention, Perception, $\&$ Psychophysics, 73(7): 2104-2123, 2011.

M. S. Livingstone and D. Hubel. Segregation of form, color, movement, and depth: anatomy, physiology, and perception. Science, 240(4853):740-749, 1988.

M. S. Livingstone and D. H. Hubel. Psychophysical evidence for separate channels for the perception of form, color, movement, and depth. Journal of Neuroscience, 7(11):3416-3468, 1987.

M. S. Livingstone, C. C. Pack, and R. T. Born. Two-dimensional substructure of MT receptive fields. Neuron, 30(3):781-793, 2001.

I. Loewenfeld. The pupil: anatomy, physiology, and clinical applications. Oxford: Butterworth-Heinemann, 1999.

O. Loewenstein. Experimentelle Beiträge zur Lehre von den katatonischen Pupillenveränderungen. Monatsschrift für Psychiatrie and Neurologie, 47: 194-215, 1920.

S. J. Luck, L. Chelazzi, S. A. Hillyard, and R. Desimone. Neural mechanisms of spatial selective attention in areas V1, V2, and V4 of macaque visual cortex. Journal of Neurophysiology, 77(1):24-42, 1997. 
E. A. Markakis, K. P. Vives, J. Bober, S. Leichtle, C. Leranth, J. Beecham, J. D. Elsworth, R. H. Roth, R. J. Samulski, and D. E. Redmond. Comparative transduction efficiency of AAV vector serotypes 1-6 in the substantia nigra and striatum of the primate brain. Molecular Therapy, 18(3):588-593, 2010 .

N. T. Markov, J. Vezoli, P. Chameau, A. Falchier, R. Quilodran, C. Huissoud, C. Lamy, P. Misery, P. Giroud, S. Ullman, P. Barone, C. Dehay, K. Knoblauch, and H. Kennedy. Anatomy of hierarchy: Feedforward and feedback pathways in macaque visual cortex. Journal of Comparative Neurology, 522(1):225-259, 2013.

R. Marois, D. J. Yi, and M. M. Chun. The neural fate of consciously perceived and missed events in the attentional blink. Neuron, 41(3):465-472, 2004.

S. Martens and B. Wyble. The attentional blink: Past, present, and future of a blind spot in perceptual awareness. Neuroscience and Biobehavioral Reviews, 34(6):947-957, 2010.

J. C. Martínez-Trujillo and S. Treue. Attentional modulation strength in cortical area MT depends on stimulus contrast. Neuron, 35(2):365-370, 2002 .

S. Mathôt, L. van der Linden, J. Grainger, and F. Vitu. The pupillary light response reveals the focus of covert visual attention. PLoS ONE, 8(10): e78168, 2013.

S. Mathôt, L. P. C. Umr, E. Dalmaijer, J. Grainger, and S. V. D. Stigchel. The pupillary light response reflects exogenous attention and inhibition of return. Journal of Vision, 14(7):1-9, 2014.

J. Mattis, K. M Tye, E. A. Ferenczi, C. Ramakrishnan, D. J. O'Shea, R. Prakash, L. A. Gunaydin, M. Hyun, L. E. Fenno, V. Gradinaru, O. Yizhar, and K. Deisseroth. Principles for applying optogenetic tools derived from direct comparative analysis of microbial opsins. Nature Methods, 9(2):159-172, 2012. 
J. H. Maunsell and D. C. Van Essen. Functional properties of neurons in middle temporal visual area of the macaque monkey. II. Binocular interactions and sensitivity to binocular disparity. Journal of Neurophysiology, 49(5): 1148-1167, 1983.

J. H. Maunsell and D. C. van Essen. The connections of the middle temporal visual area (MT) and their relationship to a cortical hierarchy in the macaque monkey. Journal of Neuroscience, 3(12):2563-2586, 1983.

J. H Maunsell, T. A. Nealey, and D. D. DePriest. Magnocellular and parvocellular contributions to responses in the middle temporal visual area (MT) of the macaque monkey. Journal of Neuroscience, 10(10):3323-3334, 1990.

J. H. R. Maunsell and D. C. Van Essen. Functional properties of neurons in the middle temporal visual area (MT) of the macaque monkey. I. Selectivity for stimulus direction, speed, and orientation. Journal of Neurophysiology, 49(5):1127-1147, 1983.

C. J. McAdams and J. H. Maunsell. Effects of attention on orientation-tuning functions of single neurons in macaque cortical area V4. Journal of Neuroscience, 19(1):431-441, 1999.

K. McAlonan, J. Cavanaugh, and R. H. Wurtz. Guarding the gateway to cortex with attention in visual thalamus. Nature, 456(7220):391-394, 2008.

N. R. McFarland, J. S. Lee, B. T. Hyman, and P. J. McLean. Comparison of transduction efficiency of recombinant AAV serotypes 1,2, 5, and 8 in the rat nigrostriatal system. Journal of Neurochemistry, 109(3):838-845, 2009.

S. P McKee and L. Welch. Sequential recruitment in the discrimination of velocity. Journal of the Optical Society of America A, 2(2):243-251, 1985.

J. P. McLean, D. E. Broadbent, and M. H. P. Broadbent. Combining attributes in rapid serial visual presentation tasks. The Quarterly Journal of Experimental Psychology Section A, 35(1):171-186, 1983.

W. H. Merigan and J. H. R. Maunsell. Macaque vision after magnocellular lateral geniculate lesions. Visual Neuroscience, 5(4):347-352, 1990. 
W. H. Merigan, C. E. Byrne, and J. H. Maunsell. Does primate motion perception depend on the magnocellular pathway? Journal of Neuroscience, 11(11):3422-3429, 1991.

Y. Merrikhi, K. Clark, E. Albarran, M. Parsa, M. Zirnsak, T. Moore, and B. Noudoost. Spatial working memory alters the efficacy of input to visual cortex. Nature Communications, 8:15041, 2017.

C. W. Mohler, M. E. Goldberg, and R. H. Wurtz. Visual receptive fields of frontal eye field neurons. Brain Research, 61:385-389, 1973.

T. Moore. Microstimulation of the frontal eye field and its effects on covert spatial attention. Journal of Neurophysiology, 91(1):152-162, 2004.

T. Moore and K. M. Armstrong. Selective gating of visual signals by microstimulation of frontal cortex. Nature, 421(6921):370-373, 2003.

T. Moore and M. Fallah. Control of eye movements and spatial attention. Proceedings of the National Academy of Sciences, 98(3):1273-1276, 2001.

J. Moran and R. Desimone. Selective attention gates visual processing in the extrastriate cortex. Science, 229(4715):782-784, 1985.

J. H. Morrison, S. L. Foote, D. O'Connor, and F. E. Bloom. Laminar, tangential and regional organization of the noradrenergic innervation of monkey cortex: Dopamine- $\beta$-hydroxylase immunohistochemistry. Brain Research Bulletin, 9(1-6):309-319, 1982.

B. C. Motter. Focal attention produces spatially selective processing in visual cortical areas V1, V2, and V4 in the presence of competing stimuli. Journal of Neurophysiology, 70(3):909-919, 1993.

A. Mueller, D. S. Hong, S. Shepard, and T. Moore. Linking ADHD to the neural circuitry of attention. Trends in Cognitive Sciences, 21(6):474-488, 2017.

P. R. Murphy, R. G. O'Connell, M. O’Sullivan, I. H. Robertson, and J. H. Balsters. Pupil diameter covaries with BOLD activity in human locus coeruleus. Human Brain Mapping, 35(8):4140-4154, 2014. 
M. Naber, G. A. Alvarez, and K. Nakayama. Tracking the allocation of attention using human pupillary oscillations. Frontiers in Psychology, 4:919, 2013.

J. J. Nassi and E. M. Callaway. Parallel processing strategies of the primate visual system. Nature Reviews Neuroscience, 10(5):360-372, 2009.

F. Newcombe and R. Russell. Dissociated visual perceptual and spatial deficits in focal lesions of the right hemisphere. Journal of Neurology, Neurosurgery and Psychiatry, 32(2):73-81, 1969.

W. Newsome, R. Wurtz, M. Dürsteler, and A. Mikami. Deficits in visual motion processing following ibotenic acid lesions of the middle temporal visual area of the macaque monkey. Journal of Neuroscience, 5(3):825-840, 1985.

W. T. Newsome and E. B. Paré. A selective impairment of motion perception following lesions of the middle temporal visual area (MT). Journal of Neuroscience, 8(6):2201-2211, 1988.

W. T. Newsome, R. H. Wurtz, and H. Komatsu. Relation of cortical areas MT and MST to pursuit eye movements. II. Differentiation of retinal from extraretinal inputs. Journal of Neurophysiology, 60(2):604-620, 1988.

R. Niebergall, P. S. Khayat, S. Treue, and J. C. Martinez-Trujillo. Expansion of MT neurons excitatory receptive fields during covert attentive tracking. Journal of Neuroscience, 31(43):15499-15510, 2011.

S. Nieuwenhuis, M. S. Gilzenrat, B. D. Holmes, and J. D. Cohen. The role of the locus coeruleus in mediating the attentional blink: A neurocomputational theory. Journal of Experimental Psychology: General, 134(3): 291-307, 2005.

S. Nieuwenhuis, I. C. Van Nieuwpoort, D. J. Veltman, and M. L. Drent. Effects of the noradrenergic agonist clonidine on temporal and spatial attention. Psychopharmacology, 193(2):261-269, 2007. 
M. R. Nieuwenstein and M. C. Potter. Temporal limits of selection and memory encoding: A comparison of whole versus partial report in rapid serial visual presentation. Psychological Science, 17(6):471-475, 2006.

T. Ninomiya, H. Sawamura, K.-I. Inoue, and M. Takada. Segregated pathways carrying frontally derived top-down signals to visual areas MT and V4 in macaques. Journal of Neuroscience, 32(20):6851-6858, 2012.

A.C. Nobre, D.R. Gitelman, E.C. Dias, and M.M. Mesulam. Covert visual spatial orienting and saccades: Overlapping neural systems. NeuroImage, 11(3):210-216, 2000.

B. Noudoost and T. Moore. Control of visual cortical signals by prefrontal dopamine. Nature, 474(7351):372-375, 2011a.

B. Noudoost and T. Moore. The role of neuromodulators in selective attention. Trends in cognitive sciences, 25(12):585-591, $2011 \mathrm{~b}$.

B. Noudoost, K. L. Clark, and T. Moore. A distinct contribution of the frontal eye field to the visual representation of saccadic targets. Journal of Neuroscience, 34(10):3687-3698, 2014.

D. H O'Connor, M. M. Fukui, M. A. Pinsk, and S. Kastner. Attention modulates responses in the human lateral geniculate nucleus. Nature Neuroscience, 5(11):1203-1209, 2002.

S. Ohayon, P. Grimaldi, N. Schweers, and D. Y. Tsao. Saccade modulation by optical and electrical stimulation in the macaque frontal eye field. Journal of Neuroscience, 33(42):16684-16697, 2013.

C. N. L. Olivers, S. Van Der Stigchel, and J. Hulleman. Spreading the sparing: Against a limited-capacity account of the attentional blink. Psychological Research, 71(2):126-139, 2007.

B. N. Oumesmar, L. Vignais, and A. Baron-Van Evercooren. Developmental expression of platelet-derived growth factor alpha-receptor in neurons and glial cells of the mouse CNS. Journal of Neuroscience, 17(1):125-139, 1997. 
J. A. Perrone and A. Thiele. Speed skills: measuring the visual speed analyzing properties of primate MT neurons. Nature Neuroscience, 4(5):526-532, 2001.

M. Petrides, G. Cadoret, and S. Mackey. Orofacial somatomotor responses in the macaque monkey homologue of broca's area. Nature, 435(7046): 1235-1238, 2005.

M. Petrides, F. Tomaiuolo, E. H. Yeterian, and D. N. Pandya. The prefrontal cortex: Comparative architectonic organization in the human and the macaque monkey brains. Cortex, 48(1):46-57, 2012.

M. A. Pinsk. Push-pull mechanism of selective attention in human extrastriate cortex. Journal of Neurophysiology, 92(1):622-629, 2004.

W. Pohl. Dissociation of spatial discrimination deficits following frontal and parietal lesions in monkeys. Journal of Comparative and Physiological Psychology, 82(2):227-239, 1973.

G. Polanczyk, M. S. De Lima, B. L. Horta, J. Biederman, and L. A. Rohde. The worldwide prevalence of ADHD: A systematic review and metaregression analysis. American Journal of Psychiatry, 164(6):942-948, 2007.

M. Poletti, M. Rucci, and M. Carrasco. Selective attention within the foveola. Nature Neuroscience, 20(10):1413-1417, 2017.

M. I. Posner. Orienting of attention. The Quarterly Journal of Experimental Psychology, 32(1):3-25, 1980.

M. C. Potter, M. M. Chun, B. S. Banks, and M. Muckenhoupt. Two attentional deficits in serial target search: The visual attentional blink and an amodal task-switch deficit. Journal of Experimental Psychology: Learning, Memory, and Cognition, 24(4):979-992, 1998.

M. C. Potter, M. Nieuwenstein, and N. Strohminger. Whole report versus partial report in RSVP sentences. Journal of Memory and Language, 58(4): 907-915, 2008. 
P. Pouget. Visual and motor connectivity and the distribution of calciumbinding proteins in macaque frontal eye field: Implications for saccade target selection. Frontiers in Neuroanatomy, 3:2, 2009.

C. M. Privitera, L. W. Renninger, T. Carney, S. Klein, and M. Aguilar. Pupil dilation during visual target detection. Journal of Vision, 10(10):1-14, 2010.

J. Rajkowski. Activation of monkey locus coeruleus neurons varies with difficulty and performance in a target detection task. Journal of Neurophysiology, 92(1):361-371, 2004.

J. E. Raymond. New objects, not new features, trigger the attentional blink. Psychological Science, 14(1):54-59, 2003.

Jane E. Raymond, Kimron L. Shapiro, and Karen M. Arnell. Temporary suppression of visual processing in an RSVP task: An attentional blink? Journal of Experimental Psychology: Human Perception and Performance, 18(3):849-860, 1992.

G. H. Recanzone and R. H. Wurtz. Effects of attention on MT and MST neuronal activity during pursuit initiation. Journal of Neurophysiology, 83 (2):777-790, 2000.

G. Rees. Modulating irrelevant motion perception by varying attentional load in an unrelated task. Science, 278(5343):1616-1619, 1997.

J. H. Reynolds and D. J. Heeger. The normalization model of attention. Neuron, 61(2):168-185, 2009.

M. Richert, T. D Albright, and B. Krekelberg. The complex structure of receptive fields in the middle temporal area. Frontiers in Systems Neuroscience, $7: 2,2013$.

G. Rizzolatti, L. Riggio, I. Dascola, and C. Umiltá. Reorienting attention across the horizontal and vertical meridians: Evidence in favor of a premotor theory of attention. Neuropsychologia, 25(1A):31-40, 1987.

D. A. Robinson and A. F. Fuchs. Eye movements evoked by stimulation of frontal eye fields. Journal of Neurophysiology, 32(5):637-648, 1969. 
C. Rock, H. Zurita, C. Wilson, and A. J. Apicella. An inhibitory corticostriatal pathway. eLife, 5:e15890, 2016.

K. S. Rockland and D. N. Pandya. Laminar origins and terminations of cortical connections of the occipital lobe in the rhesus monkey. Brain Research, 179 (1):3-20, 1979.

K. S. Rockland, J. H. Kaas, and A. Peters. Cerebral Cortex: Volume 12: Extrastriate Cortex in Primates. Springer Science \& Business Media, 2013.

H. R. Rodman and T. D. Albright. Coding of visual stimulus velocity in area MT of the macaque. Vision Research, 27(12):2035-2048, 1987.

N. E. Ross and P. Jolicoeur. Attentional blink for color. Journal of Experimental Psychology: Human Perception and Performance, 25(6):1483-1494, 1999.

A. F. Rossi, N. P. Bichot, R. Desimone, and L. G. Ungerleider. Top down attentional deficits in macaques with lesions of lateral prefrontal cortex. Journal of Neuroscience, 27(42):11306-11314, 2007.

J. S. R. Russell. An experimental investigation of eye movements. Journal of Physiology, 17(1-2):1-26, 1894.

H. Safaai, R. Neves, O. Eschenko, N. K. Logothetis, and S. Panzeri. Modeling the effect of locus coeruleus firing on cortical state dynamics and single-trial sensory processing. PNAS, 112(41):12834-12839, 2009.

A. Sahraie, M. Milders, and M. Niedeggen. Attention induced motion blindness. Vision Research, 41(13):1613-1617, 2001.

C. D. Salzman, C. M. Murasugi, K. H. Britten, and W. T. Newsome. Microstimulation in visual area MT: Effects on direction discrimination performance. Journal of Neuroscience, 12(6):2331-2355, 1992.

T. R. Sato and J. D. Schall. Effects of stimulus-response compatibility on neural selection in frontal eye field. Neuron, 38(4):637-648, 2003. 
J. D. Schall. Neuronal activity related to visually guided saccades in the frontal eye fields of rhesus monkeys: comparison with supplementary eye fields. Journal of Neurophysiology, 66(2):559-579, 1991.

J. D. Schall. Frontal Eye Fields. In: Squire LR (ed.) Encyclopedia of Neuroscience, volume 4. Oxford: Academic Press, 2009.

J. D. Schall, A. Morel, D. J. King, and J. Bullier. Topography of visual cortex connections with frontal eye field in macaque: convergence and segregation of processing streams. Journal of Neuroscience, 15(6):4464-4487, 1995.

R. L. Schnaar, R. Gerardy-Schahn, and H. Hildebrandt. Sialic acids in the brain: Gangliosides and polysialic acid in nervous system development, stability, disease, and regeneration. Physiological Reviews, 94(2):461-518, 2014.

M. A. Schoenfeld, C. Tempelmann, A. Martinez, J.-M. Hopf, C. Sattler, H.-J. Heinze, and S. A. Hillyard. Dynamics of feature binding during objectselective attention. Proceedings of the National Academy of Sciences of the United States of America, 100(20):11806-11811, 2003.

C. E. Schroeder and P. Lakatos. Low-frequency neuronal oscillations as instruments of sensory selection. Frontiers in Psychology, 32(1):9-18, 2009.

G. Sclar and R. D. Freeman. Orientation selectivity in the cat's striate cortex is invariant with stimulus contrast. Experimental Brain Research, 46(3): 457-461, 1982.

M. A. Segraves and M. E. Goldberg. Functional properties of corticotectal neurons in the monkey's frontal eye field. Journal of Neurophysiology, 58 (6):1387-1419, 1987.

E. Seidemann and W. T. Newsome. Effect of spatial attention on the responses of area MT neurons. Journal of Neurophysiology, 81(4):1783-1794, 1999.

R. Shapley, E. Kaplan, and R. Soodak. Spatial summation and contrast sensitivity of $\mathrm{X}$ and $\mathrm{Y}$ cells in the lateral geniculate nucleus of the macaque. Nature, 292(5823):543-545, 1981. 
G. Sperling and M. J. Melchner. The attention operating characteristic: examples from visual search. Science, 202(4365):315-318, 1978.

G. B. Stanton, C. J. Bruce, and M. E. Goldberg. Topography of projections to posterior cortical areas from the macaque frontal eye fields. Journal of Comparative Neurology, 353(2):291-305, 1995.

W. R. Stauffer, A. Lak, A. Yang, M. Borel, O. Paulsen, E. S. Boyden, and W. Schultz. Dopamine neuron-specific optogenetic stimulation in rhesus macaques. Cell, 166(6):1564-1571.e6, 2016.

T. H. Svensson, B. S. Bunney, and G. K. Aghajanian. Inhibition of both noradrenergic and serotonergic neurons in brain by the alpha-adrenergic agonist clonidine. Brain Research, 92(2):291-306, 1975.

J.-M. Taymans, L. H. Vandenberghe, C. Van Den Haute, I. Thiry, C. M. Deroose, L. Mortelmans, J. M. Wilson, Z. Debyser, and V. Baekelandt. Comparative analysis of adeno-associated viral vector serotypes 1, 2, 5, 7, and 8 in mouse brain. Human Gene Therapy, 18(3):195-206, 2007.

A. Thiele, K. R. Dobkins, and T. D. Albright. The contribution of color to motion processing in macaque middle temporal area. Journal of Neuroscience, 19(15):6571-6587, 1999.

K. G. Thompson. Neuronal basis of covert spatial attention in the frontal eye field. Journal of Neuroscience, 25(41):9479-9487, 2005.

J. Tigges, M. Tigges, S. Anschel, N. A. Cross, W. D. Letbetter, and R. L. McBride. Areal and laminar distribution of neurons interconnecting the central visual cortical areas 17, 18, 19, and MT in squirrel monkey saimiri. Journal of Comparative Neurology, 202(4):539-560, 1981.

J. Tigges, M. Tigges, N. A. Cross, R. L. McBride, W. D. Let-Better, and S. Anschel. Subcortical structures projecting to visual cortical areas in squirrel monkey. Journal of Comparative Neurology, 209(1):29-40, 1982.

B. Tighilet, T. Hashikawa, and E. G. Jones. Cell- and lamina-specific expression and activity-dependent regulation of type II calcium/calmodulin- 
dependent protein kinase isoforms in monkey visual cortex. Journal of Neuroscience, 18(6):2129-2146, 1998.

J. T. Ting and G. Feng. Development of transgenic animals for optogenetic manipulation of mammalian nervous system function: Progress and prospects for behavioral neuroscience. Behavioural Brain Research, 255: 3-18, 2013.

B. Tiplady, E. Bowness, L. Stien, and G. Drummond. Selective effects of clonidine and temazepam on attention and memory. Journal of Psychopharmacology, 19(3):259-265, 2005.

A. M. Treisman and G. Gelade. A feature-integration theory of attention. Cognitive Psychology, 12(1):97-136, 1980.

S. Treue and J. C. Martinez Trujillo. Feature-based attention influences motion processing gain in macaque visual cortex. Nature, 399(6736):575-579, 1999.

S. Treue and J. H. R. Maunsell. Attentional modulation of visual motion processing in cortical areas MT and MST. Nature, 382(6591):539-541, 1996.

S. Treue and J. H. R. Maunsell. Effects of attention on the processing of motion in macaque middle temporal and medial superior temporal visual cortical areas. Journal of Neurophysiology, 19(17):7591-7602, 1999.

J. M. G. Tsui and C. C. Pack. Contrast sensitivity of MT receptive field centers and surrounds. Journal of Neurophysiology, 106(4):1888-1900, 2011.

K. M. Tye and K. Deisseroth. Optogenetic investigation of neural circuits underlying brain disease in animal models. Nature Reviews Neuroscience, 13(4):251-266, 2012.

K. M. Tye, R. Prakash, S. Y. Kim, L. E. Fenno, L. Grosenick, H. Zarabi, K. R. Thompson, V. Gradinaru, C. Ramakrishnan, and K. Deisseroth. Amygdala circuitry mediating reversible and bidirectional control of anxiety. Nature, 471(7338):358-362, 2011. 
L. G. Ungerleider and R. Desimone. Cortical connections of visual area MT in the macaque. Journal of Comparative Neurology, 248(2):190-222, 1986.

L. G. Ungerleider and M. Mishkin. Two cortical visual systems. Analysis of Visual Behavior, pages 549-586, 1982.

M. Usher. The role of locus coeruleus in the regulation of cognitive performance. Science, 283(5401):549-554, 1999.

D. C. Van Essen, J. H. R. Maunsell, and J. L. Bixby. The middle temporal visual area in the macaque: myeloarchitecture, connections, functional properties and topographic organization. The Journal of Comparative Neurology, 199(3):293-326, 1981.

L. Vignais, B. N. Oumesmar, and A. B. Baron-Van Evercooren. PDGF-alpha receptor is expressed by mature neurones of the central nervous system. Neuroreport, 6(15):1993-1996, 1995.

T. A. Visser and J. L. Ohan. Data-limited manipulations of T1 difficulty modulate the attentional blink. Canadian Journal of Experimental Psychology, 61(2):102-108, 2007.

T. A. W. Visser, W. F. Bischof, and V. Di Lollo. Attentional switching in spatial and nonspatial domains: Evidence from the attentional blink. Psychological Bulletin, 125(4):458-469, 1999.

B. Wahn, D. P. Ferris, W. D. Hairston, and P. König. Pupil sizes scale with attentional load and task experience in a multiple object tracking task. PLoS ONE, 11(12):e0168087, 2016.

A. E. Walker. A cytoarchitectural study of the prefrontal area of the macaque monkey. Journal of Comparative Neurology, 73(1):59-86, 1940.

R. W. Walters, S. M. P. Yi, K. E. Brown, M. J. Welsh, J. A. Chiorini, and J. Zabner. Binding of adeno-associated virus type 5 to 2,3-linked sialic acid is required for gene transfer. Journal of Biological Chemistry, 276(23): 20610-20616, 2001. 
C.-A. Wang, S. E. Boehnke, B. J. White, and D. P. Munoz. Microstimulation of the monkey superior colliculus induces pupil dilation without evoking saccades. Journal of Neuroscience, 32(11):3629-3636, 2012.

C. Wardak. Contribution of the monkey frontal eye field to covert visual attention. Journal of Neuroscience, 26(16):4228-4235, 2006.

M. Washburn and H. C. Moises. Electrophysiological correlates of presynaptic alpha 2-receptor-mediated inhibition of norepinephrine release at locus coeruleus synapses in dentate gyrus. Journal of Neuroscience, 9(6):2131$2140,1989$.

A. Watakabe, M. Ohtsuka, M. Kinoshita, M. Takaji, K. Isa, H. Mizukami, K. Ozawa, T. Isa, and T. Yamamori. Comparative analyses of adenoassociated viral vector serotypes 1, 2, 5, 8 and 9 in marmoset, mouse and macaque cerebral cortex. Neuroscience Research, 93:144-157, 2015.

B. D. Waterhouse, H. C. Moises, and D. J. Woodward. Phasic activation of the locus coeruleus enhances responses of primary sensory cortical neurons to peripheral receptive field stimulation. Brain Research, 790(1-2):33-44, 1998.

S. M. Wierda, H. van Rijn, N. A. Taatgen, and S. Martens. Pupil dilation deconvolution reveals the dynamics of attention at high temporal resolution. Proceedings of the National Academy of Sciences, 109(22):8456-8460, 2012.

T. Womelsdorf, K. Anton-Erxleben, F. Pieper, and S. Treue. Dynamic shifts of visual receptive fields in cortical area MT by spatial attention. Nature Neuroscience, 9(9):1156-1160, 2006.

D. K. Xiao, S. Raiguel, V. Marcar, and G. A. Orban. The spatial distribution of the antagonistic surround of MT/V5 neurons. Cerebral Cortex, 7(7): 662-677, 1997.

S. M. Zeki. Representation of central visual fields in prestriate cortex of monkey. Brain Research, 14(2):271-291, 1969. 
S. M. Zeki. Functional organization of a visual area in the posterior bank of the superior temporal sulcus of the rhesus monkey. Journal of Physiology, 236(3):549-573, 1974.

H. Zeng and L. Madisen. Mouse transgenic approaches in optogenetics. Progress in Brain Research, 196:193-213, 2012.

A. Zylberberg, M. Oliva, and M. Sigman. Pupil dilation: A fingerprint of temporal selection during the attentional blink. Frontiers in Psychology, 3: 316, 2012. 


\section{Supplementary material}




\section{Manuscript 1}

\section{Antibodies}

Table S1. Primary antibodies

\begin{tabular}{llll} 
Antibody & species & dilution & vendor \\
\hline Anti-GFP & chicken & $1: 1000$ & Aves Labs, GFP-1020 \\
Anti-mCherry & rabbit & $1: 500-1000$ & Rockland, 600-401-P16 \\
Anti-NeuN & mouse & $1: 500$ & Millipore, MAB377
\end{tabular}

\section{Table S2. Secondary antibodies}

all ordered via Dianova, from Jackson ImmunoReseaarch Laboratories, Inc.

Antibody species dilution code

Anti-Chicken $\operatorname{Ig} Y(\operatorname{IgG})(\mathrm{H}+\mathrm{L}) \quad$ donkey 1:400 703-546-155

Alexa Fluor 488-conjugated AffiniPure F(ab') ${ }_{2}$ Fragment

Anti-Mouse IgG donkey 1:400 715-546-150

Alexa Fluor 488-conjugated AffiniPure F(ab') ${ }_{2}$ Fragment

Anti-Rabbit IgG $(\mathrm{H}+\mathrm{L}) \quad$ donkey $\quad 1: 400$ 711-166-152

$\mathrm{Cy}^{\mathrm{TM}} 3$-conjugated AffiniPure $\mathrm{F}\left(\mathrm{ab}{ }^{\prime}\right)_{2}$ Fragment

Anti-Mouse IgG $(\mathrm{H}+\mathrm{L}) \quad$ donkey $\quad 1: 400 \quad 715-606-150$

Alexa Fluor 647-conjugated AffiniPure F(ab') ${ }_{2}$ Fragment 


\section{Manuscript 2}

Table S3. Datafiles of neurons included in the analysis

Filename

$$
\begin{array}{ll}
\text { jah-spAtt2-hay-005-01+07 } & \text { jah-spAtt2-hay-044-01+02 } \\
\text { jah-spAtt2-hay-006-01+04 } & \text { jah-spAtt2-hay-045-01+01 } \\
\text { jah-spAtt2-hay-009-01+02 } & \text { jah-spAtt2-hay-045-01+03 } \\
\text { jah-spAtt2-hay-010-01+04 } & \text { jah-spAtt2-hay-047-01+01 } \\
\text { jah-spAtt2-hay-011-01+02 } & \text { jah-spAtt2-hay-049-01+02 } \\
\text { jah-spAtt2-hay-011-01+04 } & \text { jah-spAtt2-hay-050-01+01 } \\
\text { jah-spAtt2-hay-012-01+06 } & \text { jah-spAtt2-hay-050-01+03 } \\
\text { jah-spAtt2-hay-015-01+02 } & \text { jah-spAtt2-hay-051-01+02 } \\
\text { jah-spAtt2-hay-021-01+04 } & \text { jah-spAtt2-hay-052-01+01 } \\
\text { jah-spAtt2-hay-022-01+03 } & \text { jah-spAtt2-hay-053-01+03 } \\
\text { jah-spAtt2-hay-023-01+01 } & \text { jah-spAtt2-hay-054-01+02 } \\
\text { jah-spAtt2-hay-024-01+05 } & \text { jah-spAtt2-hay-055-01+01 } \\
\text { jah-spAtt2-hay-026-01+02 } & \text { jah-spAtt2-hay-055-01+03 } \\
\text { jah-spAtt2-hay-029-01+02 } & \text { jah-spAtt2-hay-057-01+01 } \\
\text { jah-spAtt2-hay-034-01+02 } & \text { jah-spAtt2-hay-057-01+02 } \\
\text { jah-spAtt2-hay-035-01+01 } & \text { jah-spAtt2-hay-058-01+01 } \\
\text { jah-spAtt2-hay-036-01+01 } & \text { jah-spAtt2-hay-059-01+01 } \\
\text { jah-spAtt2-hay-037-01+01 } & \text { jah-spAtt2-hay-061-01+01 } \\
\text { jah-spAtt2-hay-038-01+01 } & \\
\text { jah-spAtt2-hay-040-01+01 } & \\
\text { jah-spAtt2-hay-041-01+02 } & \\
\text { jah-spAtt2-hay-041-01+04 } & \\
\text { jah-spAtt2-hay-042-01+01 } & \\
\text { jah-spAtt2-hay-043-01+01 } &
\end{array}
$$


Table S3b. Excluded datafiles

Datafiles were excluded due to different reasons: not enough repetitions, bad isolation, or because the same cell was recorded twice with different parameters, e.g., different values of laser power.

Filename

$$
\begin{array}{ll}
\text { jah-spAtt2-hay-008-01+05 } & \text { jah-spAtt2-hay-032-01+02 } \\
\text { jah-spAtt2-hay-012-01+03 } & \text { jah-spAtt2-hay-033-01+01 } \\
\text { jah-spAtt2-hay-012-01+05 } & \text { jah-spAtt2-hay-039-01+01 } \\
\text { jah-spAtt2-hay-014-01+03 } & \text { jah-spAtt2-hay-042-01+02 } \\
\text { jah-spAtt2-hay-016-01+02 } & \text { jah-spAtt2-hay-044-01+01 } \\
\text { jah-spAtt2-hay-017-01+03 } & \text { jah-spAtt2-hay-047-01+02 } \\
\text { jah-spAtt2-hay-020-01+03 } & \text { jah-spAtt2-hay-049-01+01 } \\
\text { jah-spAtt2-hay-021-01+01 } & \text { jah-spAtt2-hay-051-01+01 } \\
\text { jah-spAtt2-hay-022-01+02 } & \text { jah-spAtt2-hay-053-01+04 } \\
\text { jah-spAtt2-hay-023-01+02 } & \text { jah-spAtt2-hay-055-01+02 } \\
\text { jah-spAtt2-hay-024-01+04 } & \text { jah-spAtt2-hay-056-01+02 } \\
\text { jah-spAtt2-hay-025-01+03 } & \text { jah-spAtt2-hay-056-01+03 } \\
\text { jah-spAtt2-hay-026-01+04 } & \text { jah-spAtt2-hay-058-01+02 } \\
\text { jah-spAtt2-hay-028-01+02 } & \text { jah-spAtt2-hay-060-01+01 } \\
\text { jah-spAtt2-hay-030-01+05 } &
\end{array}
$$


Table S4. Information about the recording sessions

For each neuron included in the analysis, the table lists the recording date, the position of the MiniMatrix's (MM) guidetube bundle in anterior-posterior (AP) and medial-lateral (ML) coordinates relative to the middle point of the recording chamber, and the days since the viral vector injection.

Cell ID date MM pos AP MM pos ML days since injection

\begin{tabular}{|c|c|c|c|c|}
\hline & & {$[\mathrm{mm}]$} & {$[\mathrm{mm}]$} & \\
\hline $005-01+07$ & 170321 & 6.6 & 0.3 & 323 \\
\hline $006-01+04$ & 170322 & 6.7 & 0.2 & 324 \\
\hline 009-01+02 & 170327 & 6.7 & 0.1 & 329 \\
\hline $010-01+04$ & 170328 & 6.6 & 0.3 & 330 \\
\hline $011-01+02$ & 170329 & 6.7 & 0.1 & 331 \\
\hline $011-01+04$ & 170329 & 6.7 & 0.1 & 331 \\
\hline $012-01+06$ & 170330 & 6.6 & 0.2 & 332 \\
\hline $015-01+02$ & 170405 & 7.0 & 0.0 & 338 \\
\hline $021-01+04$ & 170425 & 7.5 & 0.2 & 358 \\
\hline $022-01+03$ & 170426 & 6.9 & 0.2 & 359 \\
\hline $023-01+01$ & 170428 & 7.0 & 0.2 & 360 \\
\hline $024-01+05$ & 170502 & 6.6 & 0.1 & 365 \\
\hline $026-01+02$ & 170505 & 6.5 & 0.3 & 368 \\
\hline $029-01+02$ & 170615 & 7.2 & 0.5 & 409 \\
\hline $034-01+02$ & 170705 & 7.1 & 0.1 & 429 \\
\hline $035-01+01$ & 170706 & 7.1 & 0.2 & 430 \\
\hline $036-01+01$ & 170707 & 7.2 & 0.2 & 431 \\
\hline $037-01+01$ & 170717 & 7.0 & 0.2 & 441 \\
\hline $038-01+01$ & 170718 & 7.2 & 0.2 & 442 \\
\hline $040-01+01$ & 170726 & 7.7 & 0.2 & 450 \\
\hline $041-01+02$ & 170727 & 7.8 & 0.2 & 451 \\
\hline $041-01+04$ & 170727 & 7.8 & 0.2 & 451 \\
\hline
\end{tabular}


Cell ID date MM pos AP MM pos ML days since injection $[\mathrm{mm}] \quad[\mathrm{mm}]$

$\begin{array}{lllll}042-01+01 & 170728 & 7.7 & 0.2 & 452 \\ 043-01+01 & 170729 & 7.7 & 0.2 & 453 \\ 044-01+02 & 170801 & 7.8 & 0.2 & 456 \\ 045-01+01 & 170802 & 7.6 & 0.2 & 457 \\ 045-01+03 & 170802 & 7.6 & 0.2 & 457 \\ 047-01+01 & 170804 & 7.7 & 0.4 & 459 \\ 049-01+02 & 170808 & 7.0 & 0.4 & 463 \\ 050-01+01 & 170809 & 7.0 & 0.4 & 464 \\ 050-01+03 & 170809 & 7.0 & 0.4 & 464 \\ 051-01+02 & 170810 & 6.8 & 0.3 & 465 \\ 052-01+01 & 170811 & 6.6 & 0.3 & 466 \\ 053-01+03 & 170814 & 7.0 & 0.7 & 469 \\ 054-01+02 & 170815 & 7.0 & 0.7 & 471 \\ 055-01+01 & 170816 & 7.0 & 1.0 & 471 \\ 055-01+03 & 170816 & 7.0 & 1.0 & 473 \\ 057-01+01 & 170818 & 7.3 & 0.7 & 473 \\ 057-01+02 & 170818 & 7.3 & 0.7 & 474 \\ 058-01+01 & 170819 & 7.3 & 0.7 & 475 \\ 059-01+01 & 170820 & 7.3 & 0.7 & 0.7 \\ 061-01+01 & 171017 & 7.0 & & 53\end{array}$


Table S5. Recording parameters

For each neuron included in the analysis, the table lists the preferred direction in degree (0 degree was an upward movement, 90 degree a rightward movement, etc.), the receptive field position in degrees of visual angle (dva), the electrode depth as approximate distance of recording position to the dura in $\mu \mathrm{m}$, and the vertical distance between electrode tip (E) and optical fiber tip $(\mathrm{OF})$.

Cell ID pref dir RF X RF Y electrode depth distance E-OF

\begin{tabular}{|c|c|c|c|c|c|}
\hline & {$[0]$} & [dva] & [dva] & {$[\mu \mathrm{m}]$} & {$[\mu \mathrm{m}]$} \\
\hline $005-01+07$ & 225 & 9.3 & -4.7 & 6060 & 560 \\
\hline $006-01+04$ & 270 & 1.8 & -6.3 & 5375 & 675 \\
\hline 009-01+02 & 270 & -3.4 & -7.9 & 6789 & 589 \\
\hline $010-01+04$ & 90 & 4 & 4 & 8913 & 613 \\
\hline $011-01+02$ & 315 & 13.8 & -7 & 5729 & 429 \\
\hline $011-01+04$ & 45 & 6.8 & -4.2 & 6274 & 474 \\
\hline $012-01+06$ & 45 & 4.2 & -8.9 & 6706 & 406 \\
\hline $015-01+02$ & 315 & 7.4 & -4.2 & 6489 & 489 \\
\hline $021-01+04$ & 90 & 4.5 & 4.2 & 9893 & 719 \\
\hline $022-01+03$ & 45 & 6.1 & -0.3 & 9932 & 532 \\
\hline $023-01+01$ & 225 & 5.6 & 3.1 & 10330 & 330 \\
\hline $024-01+05$ & 270 & 5.1 & 0.3 & 9288 & 288 \\
\hline $026-01+02$ & 90 & 3.3 & 3.1 & 9695 & 595 \\
\hline $029-01+02$ & 315 & 2.6 & -9.6 & 7319 & 319 \\
\hline $034-01+02$ & 315 & 5.7 & -7.2 & 7225 & 425 \\
\hline $035-01+01$ & 90 & 4.4 & -0.1 & 11800 & 800 \\
\hline $036-01+01$ & 270 & 4.1 & 2.9 & 11274 & 679 \\
\hline $037-01+01$ & 225 & 3.6 & 0.4 & 10375 & 429 \\
\hline $038-01+01$ & 315 & 4.2 & 0.3 & 10367 & 367 \\
\hline $040-01+01$ & 90 & 3.5 & 5.7 & 10680 & 380 \\
\hline $041-01+02$ & 135 & 4.2 & 2.6 & 10704 & 504 \\
\hline $041-01+04$ & 90 & 2.5 & 3.6 & 10749 & 549 \\
\hline
\end{tabular}




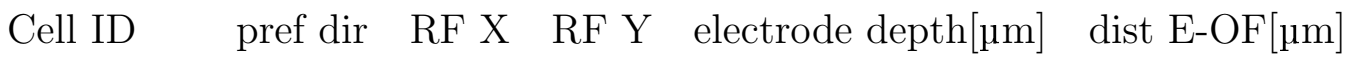

\begin{tabular}{|c|c|c|c|c|c|}
\hline & [o] & [dva] & [dva] & {$[\mu \mathrm{m}]$} & [ $\mu \mathrm{m}]$ \\
\hline $042-01+01$ & 135 & 2.7 & 2 & 10851 & 451 \\
\hline $043-01+01$ & 180 & 2.1 & 3.3 & 10827 & 227 \\
\hline $044-01+02$ & 315 & 1.5 & 4.4 & 11436 & 436 \\
\hline $045-01+01$ & 270 & -0.5 & 3.3 & 12065 & 365 \\
\hline $045-01+03$ & 45 & -1.9 & 4.5 & 12653 & 453 \\
\hline $047-01+01$ & 225 & 1.7 & 4 & 12261 & 361 \\
\hline $049-01+02$ & 90 & 4.5 & 2.9 & 12849 & 349 \\
\hline $050-01+01$ & 45 & 1.5 & 4.2 & 11985 & 465 \\
\hline $050-01+03$ & 0 & 1.7 & 2.3 & 12124 & 114 \\
\hline $051-01+02$ & 135 & 2.5 & 2.2 & 12817 & 117 \\
\hline $052-01+01$ & 225 & 3.3 & 1.5 & 12458 & 108 \\
\hline $053-01+03$ & 90 & 3.8 & -3 & 8389 & 47 \\
\hline $054-01+02$ & 90 & 2.8 & 3.2 & 12770 & 770 \\
\hline $055-01+01$ & 0 & 2.3 & 2.5 & 12803 & 182 \\
\hline $055-01+03$ & 45 & 1.8 & 3.3 & 12758 & 58 \\
\hline $057-01+01$ & 180 & 4.9 & 3.3 & 12552 & 59 \\
\hline $057-01+02$ & 180 & 3.5 & 3.7 & 12339 & 27 \\
\hline $058-01+01$ & 0 & 4 & 0.8 & 11978 & 188 \\
\hline $059-01+01$ & 90 & 2.8 & 4.7 & 12381 & 381 \\
\hline $061-01+01$ & 0 & 4.6 & -2.6 & 8700 & 600 \\
\hline
\end{tabular}




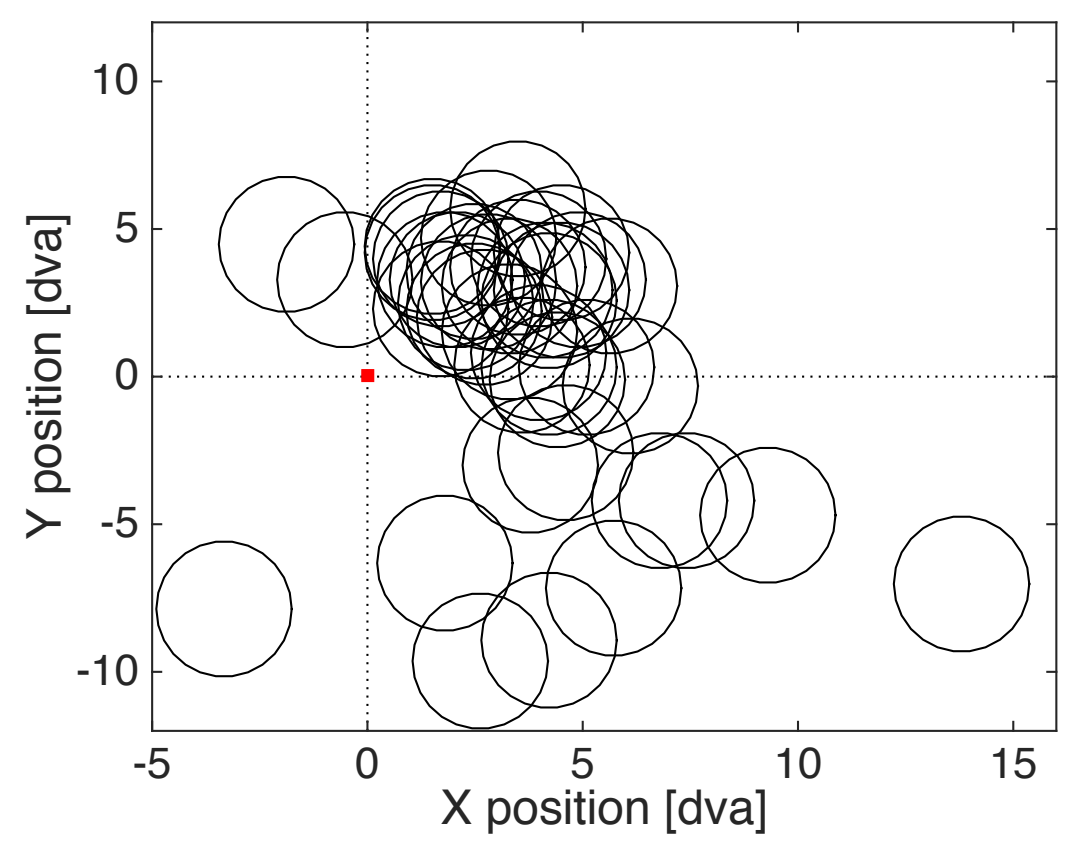

Figure 3.1: Receptive field locations of recorded neurons. Circles are not representing the size of the receptive fields. 
Table S6. Attentional index and stimulation index in time interval 300-1000ms

Cell ID AI noStim AI stim SI AttIN SI AttOUT

$\begin{array}{rrrrr}005-01+07 & 0.35 & 0.31 & -0.10 & -0.06 \\ 006-01+04 & 0.13 & 0.04 & -0.04 & 0.05 \\ 009-01+02 & 0.28 & 0.22 & -0.03 & 0.04 \\ 010-01+04 & 0.00 & 0.06 & 0.01 & -0.05 \\ 011-01+02 & 0.24 & 0.15 & -0.08 & 0.01 \\ 011-01+04 & 0.07 & -0.02 & -0.09 & 0.00 \\ 012-01+06 & 0.09 & 0.16 & 0.02 & -0.05 \\ 015-01+02 & 0.02 & 0.00 & 0.01 & 0.03 \\ 021-01+04 & 0.09 & 0.02 & -0.01 & 0.06 \\ 022-01+03 & 0.06 & 0.02 & -0.02 & 0.01 \\ 023-01+01 & 0.19 & 0.14 & -0.06 & 0.00 \\ 024-01+05 & 0.11 & 0.14 & 0.05 & 0.02 \\ 026-01+02 & 0.09 & 0.05 & -0.05 & -0.02 \\ 029-01+02 & 0.22 & 0.20 & -0.01 & 0.01 \\ 034-01+02 & 0.20 & 0.24 & 0.03 & -0.02 \\ 035-01+01 & 0.11 & 0.09 & 0.00 & 0.02 \\ 036-01+01 & 0.11 & 0.09 & 0.04 & 0.06 \\ 037-01+01 & 0.04 & -0.01 & 0.00 & 0.05 \\ 038-01+01 & 0.08 & 0.06 & 0.00 & 0.02 \\ 040-01+01 & 0.06 & 0.10 & 0.02 & -0.01 \\ 041-01+02 & 0.14 & 0.13 & 0.01 & 0.02 \\ 041-01+04 & 0.21 & 0.14 & -0.04 & 0.04 \\ 042-01+01 & 0.07 & 0.11 & 0.02 & -0.01 \\ 043-01+01 & 0.11 & 0.03 & -0.05 & 0.03 \\ 044-01+02 & 0.09 & 0.14 & 0.03 & -0.02 \\ 045-01+01 & 0.04 & 0.10 & 0.04 & -0.02 \\ 045-01+03 & 0.01 & 0.04 & 0.03 & -0.01 \\ 047-01+01 & 0.00 & 0.06 & -0.06 & -0.11 \\ 049-01+02 & 0.21 & 0.06 & -0.05 & 0.11\end{array}$




$\begin{array}{lcccc}\text { Cell ID } & \text { AI noStim } & \text { AI stim } & \text { SI AttIN } & \text { SI AttO } \\ & & & & \\ 050-01+01 & 0.13 & 0.01 & -0.05 & 0.06 \\ 050-01+03 & 0.18 & 0.00 & -0.08 & 0.10 \\ 051-01+02 & 0.13 & 0.07 & 0.01 & 0.06 \\ 052-01+01 & 0.22 & 0.19 & -0.05 & -0.03 \\ 053-01+03 & 0.19 & 0.09 & -0.10 & 0.00 \\ 054-01+02 & 0.02 & 0.04 & 0.04 & 0.02 \\ 055-01+01 & 0.18 & 0.26 & 0.06 & -0.02 \\ 055-01+03 & 0.19 & 0.14 & -0.03 & 0.03 \\ 057-01+01 & -0.01 & 0.06 & 0.01 & -0.06 \\ 057-01+02 & 0.15 & 0.18 & -0.01 & -0.05 \\ 058-01+01 & 0.06 & 0.03 & 0.01 & 0.04 \\ 059-01+01 & 0.08 & -0.05 & 0.03 & 0.10 \\ 061-01+01 & 0.13 & 0.03 & -0.01 & 0.09\end{array}$


Table S7. Attentional index and stimulation index in time interval 540-1000ms

Cell ID AI noStim AI stim SI AttIN SI AttOUT

$\begin{array}{rrrrr}005-01+07 & 0.40 & 0.34 & -0.13 & -0.07 \\ 006-01+04 & 0.15 & 0.03 & -0.04 & 0.08 \\ 009-01+02 & 0.35 & 0.23 & -0.06 & 0.07 \\ 010-01+04 & -0.04 & 0.11 & 0.04 & -0.10 \\ 011-01+02 & 0.26 & 0.17 & -0.08 & -0.01 \\ 011-01+04 & 0.13 & -0.04 & -0.10 & 0.07 \\ 012-01+06 & 0.13 & 0.12 & 0.01 & 0.02 \\ 015-01+02 & -0.02 & 0.01 & 0.03 & -0.01 \\ 021-01+04 & 0.10 & 0.01 & -0.03 & 0.06 \\ 022-01+03 & 0.07 & 0.03 & -0.03 & 0.00 \\ 023-01+01 & 0.26 & 0.21 & -0.06 & 0.00 \\ 024-01+05 & 0.14 & 0.18 & 0.07 & 0.04 \\ 026-01+02 & 0.11 & 0.03 & -0.08 & 0.00 \\ 029-01+02 & 0.21 & 0.19 & -0.02 & 0.00 \\ 034-01+02 & 0.21 & 0.23 & -0.01 & -0.03 \\ 035-01+01 & 0.13 & 0.11 & 0.02 & 0.05 \\ 036-01+01 & 0.15 & 0.10 & 0.01 & 0.07 \\ 037-01+01 & 0.09 & -0.02 & -0.02 & 0.09 \\ 038-01+01 & 0.09 & 0.06 & 0.00 & 0.03 \\ 040-01+01 & 0.09 & 0.12 & 0.03 & 0.00 \\ 041-01+02 & 0.17 & 0.14 & 0.01 & 0.04 \\ 041-01+04 & 0.26 & 0.19 & -0.02 & 0.04 \\ 042-01+01 & 0.09 & 0.13 & 0.02 & -0.02 \\ 043-01+01 & 0.10 & 0.06 & -0.04 & 0.00 \\ 044-01+02 & 0.09 & 0.14 & 0.04 & -0.01 \\ 045-01+01 & 0.03 & 0.10 & 0.02 & -0.05 \\ 045-01+03 & 0.01 & -0.01 & -0.01 & 0.01 \\ 047-01+01 & 0.01 & 0.03 & -0.07 & -0.09 \\ 049-01+02 & 0.21 & 0.07 & -0.06 & 0.08\end{array}$


Cell ID AI noStim AI stim SI AttIN SI AttOUT

$\begin{array}{rrrrr}050-01+01 & 0.17 & 0.03 & -0.09 & 0.05 \\ 050-01+03 & 0.20 & 0.00 & -0.08 & 0.13 \\ 051-01+02 & 0.16 & 0.02 & -0.06 & 0.08 \\ 052-01+01 & 0.27 & 0.20 & -0.07 & 0.01 \\ 053-01+03 & 0.22 & 0.09 & -0.12 & 0.02 \\ 054-01+02 & 0.03 & 0.03 & 0.01 & 0.01 \\ 055-01+01 & 0.20 & 0.27 & 0.07 & 0.00 \\ 055-01+03 & 0.22 & 0.14 & -0.04 & 0.04 \\ 057-01+01 & -0.05 & 0.06 & 0.04 & -0.07 \\ 057-01+02 & 0.16 & 0.22 & -0.02 & -0.08 \\ 058-01+01 & 0.06 & 0.04 & 0.00 & 0.03 \\ 059-01+01 & 0.12 & -0.03 & -0.05 & 0.10 \\ 061-01+01 & 0.14 & 0.06 & 0.00 & 0.08\end{array}$




\section{Manuscript 3}

Table S8. Subject information

Subject Gender Age Task order

$\begin{array}{llll}\text { AnR } & \text { female } & 27 & \text { ST, DT } \\ \text { BaS } & \text { male } & 27 & \text { ST, DT } \\ \text { CaZ } & \text { female } & 29 & \text { ST, DT } \\ \text { ChD } & \text { female } & 22 & \text { ST, DT } \\ \text { CvW } & \text { female } & 26 & \text { ST, DT } \\ \text { EdT } & \text { male } & 26 & \text { ST, DT } \\ \text { FaA } & \text { female } & 28 & \text { DT, ST } \\ \text { LiT } & \text { female } & 28 & \text { DT, ST } \\ \text { PhS } & \text { male } & 26 & \text { ST, DT } \\ \text { RaP } & \text { female } & 24 & \text { DT, ST } \\ \text { SaW } & \text { male } & 31 & \text { DT, ST } \\ \text { ShP } & \text { male } & 26 & \text { DT, ST }\end{array}$

excluded subjects (i.e, they did not reach the predefined performance to participate in the recording):

FrM

LaN

MiM

StW 
Table S9. Subject information

Subject Gender Age Task order

BaS male 27 DT, ST

ChG female 24 ST, DT

$\mathrm{CvW}$ female 26 ST, DT

EdT male 26 DT, ST

FaA female 28 ST, DT

FaS male 21 ST, DT

LiT female 28 ST, DT

$\mathrm{PaG}$ female 29 DT, ST

$\mathrm{PhS}$ male 26 DT, ST

RaP female 25 DT, ST

SaW male 31 DT, ST

SbP male 28 ST, DT

excluded subjects (i.e, they did not reach the predefined performance to participate in the recording):
$\mathrm{AlJ}$
AnE
AnR
$\mathrm{CaZ}$
$\mathrm{MaG}$
AyM 
Table S10. Subject information

Subject Gender Age Task order

$\begin{array}{llll}\text { BaS } & \text { male } & 28 & \text { ST, DT } \\ \text { CaZ } & \text { female } & 30 & \text { ST, DT } \\ \text { CvW } & \text { female } & 27 & \text { DT, ST } \\ \text { FaA } & \text { female } & 28 & \text { ST, DT } \\ \text { FaS } & \text { female } & 21 & \text { ST, DT } \\ \text { FaV } & \text { male } & 29 & \text { DT, ST } \\ \text { FlH } & \text { female } & 24 & \text { DT, ST } \\ \text { IsF } & \text { female } & 20 & \text { DT, ST } \\ \text { JoS } & \text { male } & 31 & \text { DT, ST } \\ \text { KrD } & \text { female } & 24 & \text { ST, DT } \\ \text { SbP } & \text { male } & 29 & \text { DT, ST } \\ \text { SaW } & \text { male } & 32 & \text { ST, DT }\end{array}$

excluded subjects (i.e, they did not reach the predefined performance to participate in the recording):

ChL

$\mathrm{FaH}$

$\mathrm{JaG}$

$\mathrm{KaB}$

$\mathrm{MeD}$

$\mathrm{PaG}$ 
Table S11. Subject information

Subject Gender Age Task order

BaS male 28 ST, DT

CaZ female 30 DT, ST

ChG female 25 DT, ST

ChL female 29 ST, DT

CvW female 27 ST, DT

FaA female 28 ST, DT

FlK male 20 DT, ST

IsF female 21 DT, ST

JeS male 22 ST, DT

JoS male 31 DT, ST

MaM female 25 DT, ST

SaW male 31 ST, DT

excluded subjects (i.e, they did not reach the predefined performance to participate in the recording):

$\mathrm{AKH}$

AKR

AyM

$\mathrm{FaH}$

$\mathrm{FaS}$

LoS

$\mathrm{MeD}$

WiR 
Table S12. Datafiles of subjects included in analysis

Subject Task Filename

$\begin{array}{lll}\text { AnR } & \text { single-task } & \text { JaH_ABRDP_ST_AnR_7.mwk } \\ \text { BaS } & \text { single-task } & \text { JaH_ABRDP_ST_BaS_2.mwk } \\ \text { CaZ } & \text { single-task } & \text { JaH_ABRDP_ST_CaZ_5.mwk } \\ \text { ChD } & \text { single-task } & \text { JaH_ABRDP_ST_ChD_6.mwk } \\ \text { CvW } & \text { single-task } & \text { JaH_ABRDP_ST_CvW_1.mwk } \\ \text { EdT } & \text { single-task } & \text { JaH_ABRDP_ST_EdT_10.mwk } \\ \text { FaA } & \text { single-task } & \text { JaH_ABRDP_ST_FaA_13.mwk } \\ \text { LiT } & \text { single-task } & \text { JaH_ABRDP_ST_LiT_9.mwk } \\ \text { PhS } & \text { single-task } & \text { JaH_ABRDP_ST_PhS_8.mwk } \\ \text { RaP } & \text { single-task } & \text { JaH_ABRDP_ST_RaP_4.mwk } \\ \text { SaW } & \text { single-task } & \text { JaH_ABRDP_ST_SaW_3.mwk } \\ \text { ShP } & \text { single-task } & \text { JaH_ABRDP_ST_ShP_11.mwk } \\ & & \\ \text { AnR } & \text { dual-task } & \text { JaH_ABRDP_DT_AnR_7.mwk } \\ \text { BaS } & \text { dual-task } & \text { JaH_ABRDP_DT_BaS_2.mwk } \\ \text { CaZ } & \text { dual-task } & \text { JaH_ABRDP_DT_CaZ_5.mwk } \\ \text { ChD } & \text { dual-task } & \text { JaH_ABRDP_DT_ChD_6.mwk } \\ \text { CvW } & \text { dual-task } & \text { JaH_ABRDP_DT_CvW_1.mwk } \\ \text { EdT } & \text { dual-task } & \text { JaH_ABRDP_DT_EdT_10.mwk } \\ \text { FaA } & \text { dual-task } & \text { JaH_ABRDP_DT_FaA_13.mwk } \\ \text { LiT } & \text { dual-task } & \text { JaH_ABRDP_DT_LiT_9.mwk } \\ \text { PhS } & \text { dual-task } & \text { JaH_ABRDP_DT_PhS_8.mwk } \\ \text { RaP } & \text { dual-task } & \text { JaH_ABRDP_DT_RaP_4.mwk } \\ \text { SaW } & \text { dual-task } & \text { JaH_ABRDP_DT_SaW_3.mwk } \\ \text { ShP } & \text { dual-task } & \text { JaH_ABRDP_DT_ShP_11.mwk }\end{array}$


Table S13. Datafiles of subjects included in analysis

Subject Task Filename

$\begin{array}{lll}\text { BaS } & \text { single-task } & \text { JaH_AB_RDP_V2_ST_BaS_5.mwk } \\ \text { ChG } & \text { single-task } & \text { JaH_AB_RDP_V2_ST_ChG_11.mwk } \\ \text { CvW } & \text { single-task } & \text { JaH_AB_RDP_V2_ST_CvW_1.mwk } \\ \text { EdT } & \text { single-task } & \text { JaH_AB_RDP_V2_ST_EdT_6.mwk } \\ \text { FaA } & \text { single-task } & \text { JaH_AB_RDP_V2_ST_FaA_10.mwk } \\ \text { FaS } & \text { single-task } & \text { JaH_AB_RDP_V2_ST_FaS_01082012.mwk } \\ \text { LiT } & \text { single-task } & \text { JaH_AB_RDP_V2_ST_LiT_7.mwk } \\ \text { PaG } & \text { single-task } & \text { JaH_AB_RDP_V2_ST_PaG_03082012.mwk } \\ \text { PhS } & \text { single-task } & \text { JaH_AB_RDP_V2_ST_PhS_9.mwk } \\ \text { RaP } & \text { single-task } & \text { JaH_AB_RDP_V2_ST_RaP_4.mwk } \\ \text { SbP } & \text { single-task } & \text { JaH_AB_RDP_V2_ST_SbP_8.mwk } \\ \text { SaW } & \text { single-task } & \text { JaH_AB_RDP_V2_ST_SaW_2.mwk } \\ & & \\ \text { BaS } & \text { dual-task } & \text { JaH_AB_RDP_V2_DT_BaS_5.mwk } \\ \text { ChG } & \text { dual-task } & \text { JaH_AB_RDP_V2_DT_ChG_11.mwk } \\ \text { CvW } & \text { dual-task } & \text { JaH_AB_RDP_V2_DT_CvW_1.mwk } \\ \text { EdT } & \text { dual-task } & \text { JaH_AB_RDP_V2_DT_EdT_6.mwk } \\ \text { FaA } & \text { dual-task } & \text { JaH_AB_RDP_V2_DT_FaA_10.mwk } \\ \text { FaS } & \text { dual-task } & \text { JaH_AB_RDP_V2_DT_FaS_01082012.mwk } \\ \text { LiT } & \text { dual-task } & \text { JaH_AB_RDP_V2_DT_LiT_7.mwk } \\ \text { PaG } & \text { dual-task } & \text { JaH_AB_RDP_V2_DT_PaG_03082012.mwk } \\ \text { PhS } & \text { dual-task } & \text { JaH_AB_RDP_V2_DT_PhS_9.mwk } \\ \text { RaP } & \text { dual-task } & \text { JaH_AB_RDP_V2_DT_RaP_4.mwk } \\ \text { SaW } & \text { dual-task } & \text { JaH_AB_RDP_V2_DT_SaW_2.mwk } \\ \text { SbP } & \text { dual-task } & \text { JaH_AB_RDP_V2_DT_SbP_8.mwk } \\ & & \end{array}$


Table S14. Datafiles of subjects included in analysis

Subject Task Filename

$\begin{array}{lll}\text { BaS } & \text { single-task } & \text { JaH_AB_LetterRDP_ST_BaS_8.mwk } \\ \text { CaZ } & \text { single-task } & \text { JaH_AB_LetterRDP_ST_CaZ_6.mwk } \\ \text { CrW } & \text { single-task } & \text { JaH_AB_LetterRDP_ST_CvW_14.mwk } \\ \text { FaA } & \text { single-task } & \text { JaH_AB_LetterRDP_ST_FaA_2.mwk } \\ \text { FaS } & \text { single-task } & \text { JaH_AB_LetterRDP_ST_FaS_3.mwk } \\ \text { FaV } & \text { single-task } & \text { JaH_AB_LetterRDP_ST_FaV_17.mwk } \\ \text { FlH } & \text { single-task } & \text { JaH_AB_LetterRDP_ST_FlH_4.mwk } \\ \text { IsF } & \text { single-task } & \text { JaH_AB_LetterRDP_ST_IsF_5.mwk } \\ \text { JoS } & \text { single-task } & \text { JaH_AB_LetterRDP_ST_JoS_1.mwk } \\ \text { KrD } & \text { single-task } & \text { JaH_AB_LetterRDP_ST_KrD_15.mwk } \\ \text { SaW } & \text { single-task } & \text { JaH_AB_LetterRDP_ST_SaW_12.mwk } \\ \text { SbP } & \text { single-task } & \text { JaH_AB_LetterRDP_ST_SbP_9.mwk } \\ & & \\ \text { BaS } & \text { dual-task } & \text { JaH_AB_LetterRDP_DT_BaS_8.mwk } \\ \text { CaZ } & \text { dual-task } & \text { JaH_AB_LetterRDP_DT_CaZ_6.mwk } \\ \text { CvW } & \text { dual-task } & \text { JaH_AB_LetterRDP_DT_CvW_14.mwk } \\ \text { FaA } & \text { dual-task } & \text { JaH_AB_LetterRDP_DT_FaA_2.mwk } \\ \text { FaS } & \text { dual-task } & \text { JaH_AB_LetterRDP_DT_FaS_3.mwk } \\ \text { FaV } & \text { dual-task } & \text { JaH_AB_LetterRDP_DT_FaV_17.mwk } \\ \text { FlH } & \text { dual-task } & \text { JaH_AB_LetterRDP_DT_FlH_4.mwk } \\ \text { IsF } & \text { dual-task } & \text { JaH_AB_LetterRDP_DT_IsF_5.mwk } \\ \text { JoS } & \text { dual-task } & \text { JaH_AB_LetterRDP_DT_JoS_1.mwk } \\ \text { KrD } & \text { dual-task } & \text { JaH_AB_LetterRDP_DT_KrD_15.mwk } \\ \text { SaW } & \text { dual-task } & \text { JaH_AB_LetterRDP_DT_SaW_12.mwk } \\ \text { SbP } & \text { dual-task } & \text { JaH_AB_LetterRDP_DT_SbP_9.mwk }\end{array}$


Table S15. Datafiles of subjects included in analysis

Subject Task Filename

$\begin{array}{lll}\text { BaS } & \text { single-task } & \text { JES_AB_RDPLetter_ST_BaS_10.mwk } \\ \text { CaZ } & \text { single-task } & \text { JaH_AB_RDPLetter_ST_CaZ_19.mwk } \\ \text { ChG } & \text { single-task } & \text { JES_AB_RDPLetter_ST_ChG_11.mwk } \\ \text { ChL } & \text { single-task } & \text { JaH_AB_RDPLetter_ST_ChL_16.mwk } \\ \text { CvW } & \text { single-task } & \text { JES_AB_RDPLetter_ST_CvW_12.mwk } \\ \text { FaA } & \text { single-task } & \text { JaH_AB_RDPLetter_ST_FaA_14.mwk } \\ \text { FlK } & \text { single-task } & \text { JES_AB_RDPLetter_ST_FlK_9.mwk } \\ \text { IsF } & \text { single-task } & \text { JaH_AB_RDPLetter_ST_IsF_20.mwk } \\ \text { JES } & \text { single-task } & \text { JES_AB_RDPLetter_ST_JES_7.mwk } \\ \text { JoS } & \text { single-task } & \text { JaH_AB_RDPLetter_ST_JoS_18.mwk } \\ \text { MaM } & \text { single-task } & \text { JES_AB_RDPLetter_ST_MaM_8.mwk } \\ \text { SaW } & \text { single-task } & \text { JES_AB_RDPLetter_ST_SaW_3.mwk }\end{array}$

BaS dual-task JES_AB_RDPLetter_DT_BaS_10.mwk

CaZ dual-task JaH_AB_RDPLetter_DT_CaZ_18.mwk

ChG dual-task JES_AB_RDPLetter_DT_ChG_11.mwk

ChL dual-task JaH_AB_RDPLetter_DT_ChL_16.mwk

CvW dual-task JES_AB_RDPLetter_DT_CvW_12.mwk

FaA dual-task JaH_AB_RDPLetter_DT_FaA_14.mwk

FlK dual-task JES_AB_RDPLetter_DT_FlK_9.mwk

IsF dual-task JaH_AB_RDPLetter_DT_IsF_20.mwk

JES dual-task JES_AB_RDPLetter_DT_JES_7.mwk

JoS dual-task JaH_AB_RDPLetter_DT_JoS_18.mwk

MaM dual-task JES_AB_RDPLetter_DT_MaM_8.mwk

SaW dual-task JES_AB_RDPLetter_DT_SaW_3.mwk 


\section{Manuscript 4}

\section{Table S16. Subject information}

Subject Gender Age Handedness Task order

$\begin{array}{lllll}\text { AnS } & \text { female } & 29 & \text { right } & \text { ST, DT } \\ \text { DeM } & \text { female } & 20 & \text { right } & \text { ST, DT } \\ \text { FeS } & \text { male } & 22 & \text { right } & \text { ST, DT } \\ \text { FiW } & \text { female } & 21 & \text { right } & \text { ST, DT } \\ \text { FlR } & \text { male } & 22 & \text { right } & \text { ST, DT } \\ \text { GeR } & \text { male } & 24 & \text { right } & \text { ST, DT } \\ \text { LeD } & \text { male } & 21 & \text { left } & \text { ST, DT } \\ \text { LiT } & \text { female } & 33 & \text { right } & \text { ST, DT } \\ \text { MaR } & \text { male } & 26 & \text { right } & \text { ST, DT } \\ \text { MeB } & \text { female } & 24 & \text { right } & \text { ST, DT } \\ \text { MrH } & \text { male } & 19 & \text { right } & \text { ST, DT } \\ \text { OaS } & \text { male } & 45 & \text { right } & \text { ST, DT } \\ \text { ThM } & \text { female } & 24 & \text { right } & \text { ST, DT } \\ \text { TiM } & \text { male } & 27 & \text { right } & \text { ST, DT }\end{array}$

excluded subjects (i.e, they did not reach the predefined performance to participate in the recording):

$\begin{array}{ll}\text { AaB } & \text { LoD } \\ \text { ArB } & \text { LrB } \\ \text { HaJ } & \text { MaV } \\ \text { JeM } & \text { OsC } \\ \text { JuF } & \text { RoD } \\ \text { JuS } & \text { SxS }\end{array}$


Table S17. Datafiles of subjects included in analysis

Subject Task Filename

$\begin{array}{lll}\text { AnS } & \text { single-task } & \text { DwT_v3.4.2_AnS_T1_2_83_R12_1.mwk } \\ \text { DeM } & \text { single-task } & \text { DwT_v3.4.2_DeM_T1_2_83_R12_2.mwk } \\ \text { FeS } & \text { single-task } & \text { DwT_v3.4.2_FeS_T1_2_83_R12_2.mwk } \\ \text { FiW } & \text { single-task } & \text { DwT_v3.4.2_FiW_T1_1_83_R12_5.mwk } \\ \text { FlR } & \text { single-task } & \text { DwT_v3.4.2_FlR_T1_2_83_R12_1.mwk } \\ \text { GeR } & \text { single-task } & \text { DwT_v3.4.2_GeR_T1_2_83_R12_2.mwk } \\ \text { LeD } & \text { single-task } & \text { DwT_v3.4.2_LeD_T1_1_83_R12_1.mwk } \\ \text { LiT } & \text { single-task } & \text { DwT_v3.4.2_LiT_T1_1_83_R12_1.mwk } \\ \text { MaR } & \text { single-task } & \text { DwT_v3.4.2_MaR_T1_1_83_R12_2.mwk } \\ \text { MeB } & \text { single-task } & \text { DwT_v3.4.2_MeB_T1_2_83_R12_1.mwk } \\ \text { MrH } & \text { single-task } & \text { DwT_v3.4.2_MrH_T1_1_83_R12_2.mwk } \\ \text { OaS } & \text { single-task } & \text { DwT_v3.4.2_OaS_T1_1_83_R12_1.mwk } \\ \text { ThM } & \text { single-task } & \text { DwT_v3.4.2_ThM_T1_2_83_R12_1.mwk } \\ \text { TiM } & \text { single-task } & \text { DwT_v3.4.2_TiM_T1_1_83_R12_3.mwk } \\ & & \\ \text { AnS } & \text { dual-task } & \text { DwT_v3.4.2_AnS_DT_T2F_83_R12_1.mwk } \\ \text { DeM } & \text { dual-task } & \text { DwT_v3.4.2_DeM_DT_T2F_83_R12_1.mwk } \\ \text { FeS } & \text { dual-task } & \text { DwT_v3.4.2_FeS_DT_T2F_83_R12_1.mwk } \\ \text { FiW } & \text { dual-task } & \text { DwT_v3.4.2_FiW_DT_T2F_83_R12_1.mwk } \\ \text { FlR } & \text { dual-task } & \text { DwT_v3.4.2_FlR_DT_T2F_83_R12_1.mwk } \\ \text { GeR } & \text { dual-task } & \text { DwT_v3.4.2_GeR_DT_T2F_83_R12_1.mwk } \\ \text { LeD } & \text { dual-task } & \text { DwT_v3.4.2_LeD_DT_T2F_83_R12_1.mwk } \\ \text { LiT } & \text { dual-task } & \text { DwT_v3.4.2_LiT_DT_T2F_83_R12_1.mwk } \\ \text { MaR } & \text { dual-task } & \text { DwT_v3.4.2_MaR_DT_T2F_83_R12_1.mwk } \\ \text { MeB } & \text { dual-task } & \text { DwT_v3.4.2_MeB_DT_T2F_83_R12_1.mwk } \\ \text { MrH } & \text { dual-task } & \text { DwT_v3.4.2_MrH_DT_T2F_83_R12_1.mwk } \\ \text { OaS } & \text { dual-task } & \text { DwT_v3.4.2_OaS_DT_T2F_83_R12_1.mwk } \\ \text { ThM } & \text { dual-task } & \text { DwT_v3.4.2_ThM_DT_T2F_83_R12_1.mwk } \\ \text { TiM } & \text { dual-task } & \text { DwT_v3.4.2_TiM_DT_T2F_83_R12_1.mwk } \\ & & \end{array}$



